\title{
COMPILATION OF REQUESTS FOR NUCLEAR DATA
}

\author{
COMPILED AND EDITED BY THE NATIONAL NUCLEAR DATA CENTER \\ FOR THE \\ DOE NUCLEAR DATA COMMITTEE
}

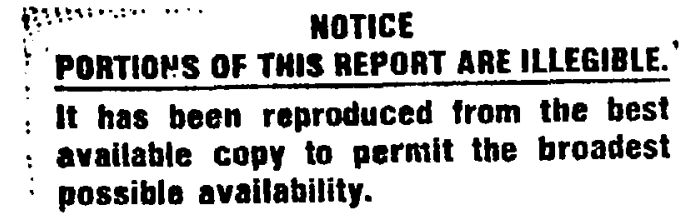

MOTICE

PORTIONS OF THIS REPOAT ARE

available copy to permit the broadest

possible availability.

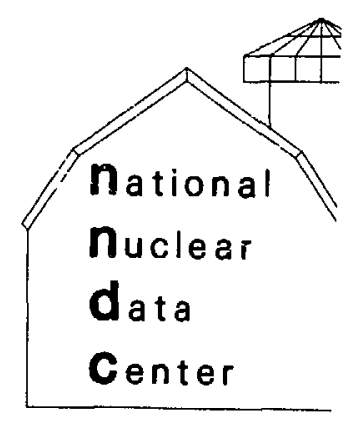

\section{DISCLAIMER}

This report was prepared as an account of work sponsored by an agency of the United States employees, makes any warranty, express or implied, nor any agency thereof, nor any of their bility for the accuracy, completeness, or implied, or assumes any legal liability or responsiprocess discloed or ence herein to , or represents that its use would not infringe privately owned rights. Reference herein to any specific commercial product, process, or service by trade name, trademark, manufacturer, or otherwise does not necessarily constitute or imply its endorsement, rocommendation, or favoring by the Unitod States Gonstitute or imply its endorsement, rocomand opinions of authors expressed herein do not necessarily state or reflect those of the United States Government or any agency thercof.

January 1983

NATIONAL NUCLEAR DATA CENTER

BROOKHAVEN NATIONAL LABORATORY ASSOCIATED UNIVERSITIES, INC.

UNDER CONTRACT NO. DE-ACO2-76CHOOO16 WITH THE

UNITED STATES DEPARTMENT OF ENERGY 


\section{DISCLAIMER}

This report was prepared as an account of work sponsored by an agency of the United States Covernment. Neither the United States Governmen nor any agency thereof, nor any of their employees, nor any of their contractors, subcontractors, or their employees, makes any warranly. express or implied, or assumes any legal liability or responsibility for the accuracy. completeness or usefulness of any information. apparalus, product or process disclosed, of represents that its use would nol infringe privalely ouned rights. Reference herein lo any specific commercial product, process. or service by trade name, trademark. manufacturer, or otherwise does not necessarily constitute or imply its endorsement, recommendation, or favoring by the United States Government or any agency. contractor or subcontractor thereof. The views and opinions of authors expressed herein do not necessarily state or reflect those of the United States Government or any agency. con-

tractor or subcontractor thereof.

$$
\begin{gathered}
\text { Printed in the United States of America } \\
\text { Available from } \\
\text { National Technical information Service } \\
\text { U. S. Department of Commerce } \\
5285 \text { Port Royal. Rond } \\
\text { Springrield. VA } 22161
\end{gathered}
$$

NTIS price codes:

Printed Copy: A05; Microfiche Copy: 101 
TABLE OF CONTENTS

page

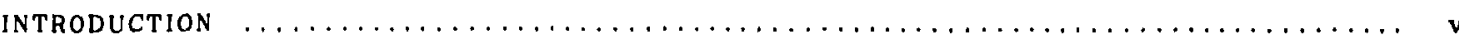

PROCEDURES FOR SUBMITTING REQUESTS $\ldots \ldots \ldots \ldots \ldots \ldots \ldots \ldots \ldots \ldots \ldots \ldots \ldots$

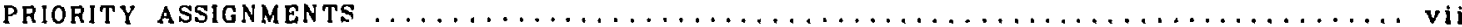

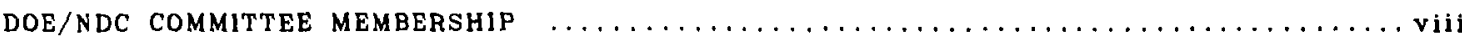

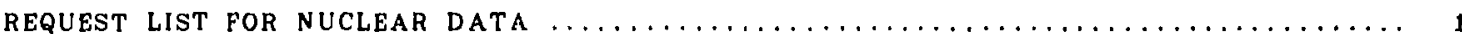

Appendices

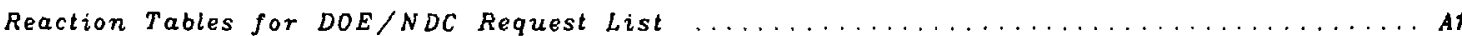

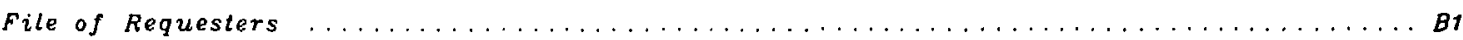

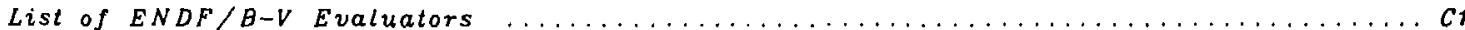




\title{
I. INTRODUCTION
}

The purpose of this compilation is to summarize the current needs of U. S. Nuclear Energy programs and other applied technologies ror nuclear data. It is the result of a biennial review in which the Department or Energy (DOE) and contractors, Department of Defense Laboratories and contractors, and other interested groups have been asked to review and revise their requests for nuclear data. It was felt that the evaluators of cross section data and the users of these evaluations should be involved in the review of the data requests to make this compilation more useful. Hence, the following review procedure was adopted for the current edition of the Data Request List; retrievals of requests from the 1981 edition pertaining to the rollowing rour categories of reviewers were sent for comments to:

\author{
1. Originators of data requests \\ 2. Evaluators of ENDF/B-V \\ 3. Members of the Data Testing Subcommittee of the \\ Cross Section Evaluation Working Group (CSEWG).
}

4. Office of Fusion Energy (OFE) data needs reviewers.

Out of a group of 106, some 60 replied with their comments and suggestions. These included recommendations for deleting requests which had been satisfied by recent measurements or evaluations. The changing priorities of some applied programs also suggested the omission of some requests and the inclusion of new ones. New data requests had to include a written justification. The revision of OFE data requests was coordinated by E. T. Cheng (GA). The comments and new requests received from all the reviewers were scrutinized by the Data Requests and Status Subcommittee of CSEWG and a preliminary Request List based on its recommendations adopted. This was further reviewed by the Data Testing and Applications Committee of CSEWG and changes in the Request List recommended by it were included.

Because of the changing and continuing character of the need for the data request information as well as the probability that current measurements may satisfy a portion of the requests, this report is to be regarded as a working document. In fact, it is maintained on a continuous access basis as a data base by the National Nuclear Data Center (NNDC).

The present request list is produced from a computerized data file by the NNDC. The system permits full use of upper case, lower case, Greek letters, subscripts and superscripts. The contents of this document, except for requests withheld by sponsoring organizations, will be included in the forthcoming edition of whEND, published by the International Atomic Energy Agency. 
This request list is ordered by target nucleus (Isotope) and then reaction type (Quantity). An attempt has been made to describe the Quantity in standard notation. In appendix A, the user will find a glossary of these symbols with a short explanatory text.

Each request is assigned a unique identifying number. The first two digits of this number give the year the request was initiated. All requests for a given Isotope and Quantity are grouped (or blocked) together The requests in a block are rollowed by any status comments.

Each request has a unique lsotope, Quantity and Requester. The requester is identified by laboratory, last name, and sponsoring U. S. government agency, e.g. BET, DEL, DNR. All requesters, together with their addresses and phone numbers, are given in appendix B. A list of the evaluators responsible for ENDF/B-V evaluations with their affiliation appears in appendix C.

All requests must give the energy (or range of energy) ror the incident particle when appropriate. The accuracy needed in percent is also given. The error quoted is assumed to be $1-\sigma$ at each measured point in the energy range requested unless a comment specifies otherwise. Sometimes a range of accuracy indicated by two values is given or some statement is given in the free text comments. An incident particle energy resolution in percent is sometimes given.

\section{PROCEDURES FOR SUBMITTING REQUESTS}

New data requests should be submitted to the designated representative who will coordinate the requests originating from each laboratory and do an initial screening. If your institution does not have a designated representative, requests may be submitted directly to the NNDC. Data requests should include a justification of the request and should state its importance and application.

In accepting request for nuclear data, the DOE/NDC assumes that the originator or the sponsoring agency has established that the existing data base has been reviewed and determined to be inadequate. In this regard, comments on evaluations that may be necessary, or the need for results from rurther theoretical work, should be included in the request. Originators of data requests must be realistic and exercise good sense and moderation, as well as a sense of proportion. in assembling their list. Requests should be ordered in terms of priority, in consultation with the program sponsors, to judge the importance of requests from the viewpoint of the whole program. From this list, a few of the most important requests should be chosen and submitted for the Data Request Compilation. This will prevent the Compilation from becoming an exercise in wishrul thinking in which most of the requests do not stand an appreciable chance of ever being fulfilled.

A. B. Smith (ANL), R. E. Chrien (BNL), R. E. Schenter (HEDL), R. Heath (INEL), P, G. Young (LANL), R. Haight (LLNL) and F. G. Perey (ORNL) 


\section{I. PRIORITY ASSIGNMENTS}

The exact meaning of priority is difficult to assess but the following definitions, which closely resemble those used by both NEANDC and the NEACRP, have been adopted by the DOE/NDC.

PRIORITY I.

PRIORITY II.

PRIORITY III.
Nuclear data which satisfy the criteria of Priority 11 and which have been selected for maximum practicable attention taking into account the urgency of nuclear energy program requirements.

Nuclear data that will be required during the next few years in the applied nuclear energy programs (for example, data needed to make the best use of reactor fuel and construction materials such as neutron moderators, absorbers, and radiation shields, space and bio-medical applications, data required for better understanding of some significant aspect or reactor behavior).

Nuclear data of more general interest and data required to fill out the body of information needed for nuclear technology.

-For example, the NEACRP assigns its highest priorities for reactor measurements as follows:

The highest priority should be given to requests for nuclear data for reactors to be built in the near future if these data are still necessary to predict the different reactor properties after all information from integral experiments and operating reactors has been used, or information on an important reactor parameter is in principle attainable through mathematical calculations from nuclear data only, or these data are needed for materials required in reactor physics measurements. 


\section{DOE/NDC COMMITTEE MEMBERSHIP}

Dr. John D. Anderson/

Dr. R. C. Haight

Physics Dept., E Division

Lawrence Livermore National

Laboratory

Livermore, CA 945.50

Dr. Russell L. Heath

Research \& Engineering

Idaho National Engineering

Laboratury

Idaho Falls, ID 93401

Dr. Richard E. Schenter

Hanford Engineering Development

Laboratory

P. D. Box 1970

Richland, WA 99352

Dr. O. A. Wasson

Radiation Physics Building

National Bureau of Standards

Washington, DC 20234

Dr, Sol Pearlstein

(Ex-officio member)

Director, NNDC

Brookhaven National Laboratory

Upton, NY 11973
Mr. Sam E. Berk

Office of Energy Research

Reactors \& Systems Radiation Branch

U. S. Department of Energy

Hashington, DC 20545

Mr. Philip B. Hemmig

Chief, Physics Branch

Reactor Research \& Technilogy

office of Energy Technology

U. S. Department of Energy

Washington, DC 20545

Dr. Alan B. Smith

Applied Physics Division

Argonne National Laboratory

Argonne, lL 60439

Dr. Stanley L. Whetstone (Chairman)

Division of Nuclear Sciences

Office of Basic Energy Sciences

U. S. Department of Energy

Washington, DC 20545
Dr. Robert E. Chrien

Physics Department

Brookhaven National Laboratory

Upton, NY 11973

Dr. Francis G. Perey

Neutron Physics Division

Oak Ridge National Laboratory

Oak Ridge, TN 37830

Dr. Philip M. Stone

Applied Plasma Physics

Orfice of Fusion Energy

U. S. Department of Energy

Washinet on, DC 20545

Dr. Phillip G. Young

T-2 Nuclear Data

Los Alamos Scientific Laboratory

Los Alamos, NM 87545 
DOE-NDC Compilation of Requests for Nuclear Data 1982

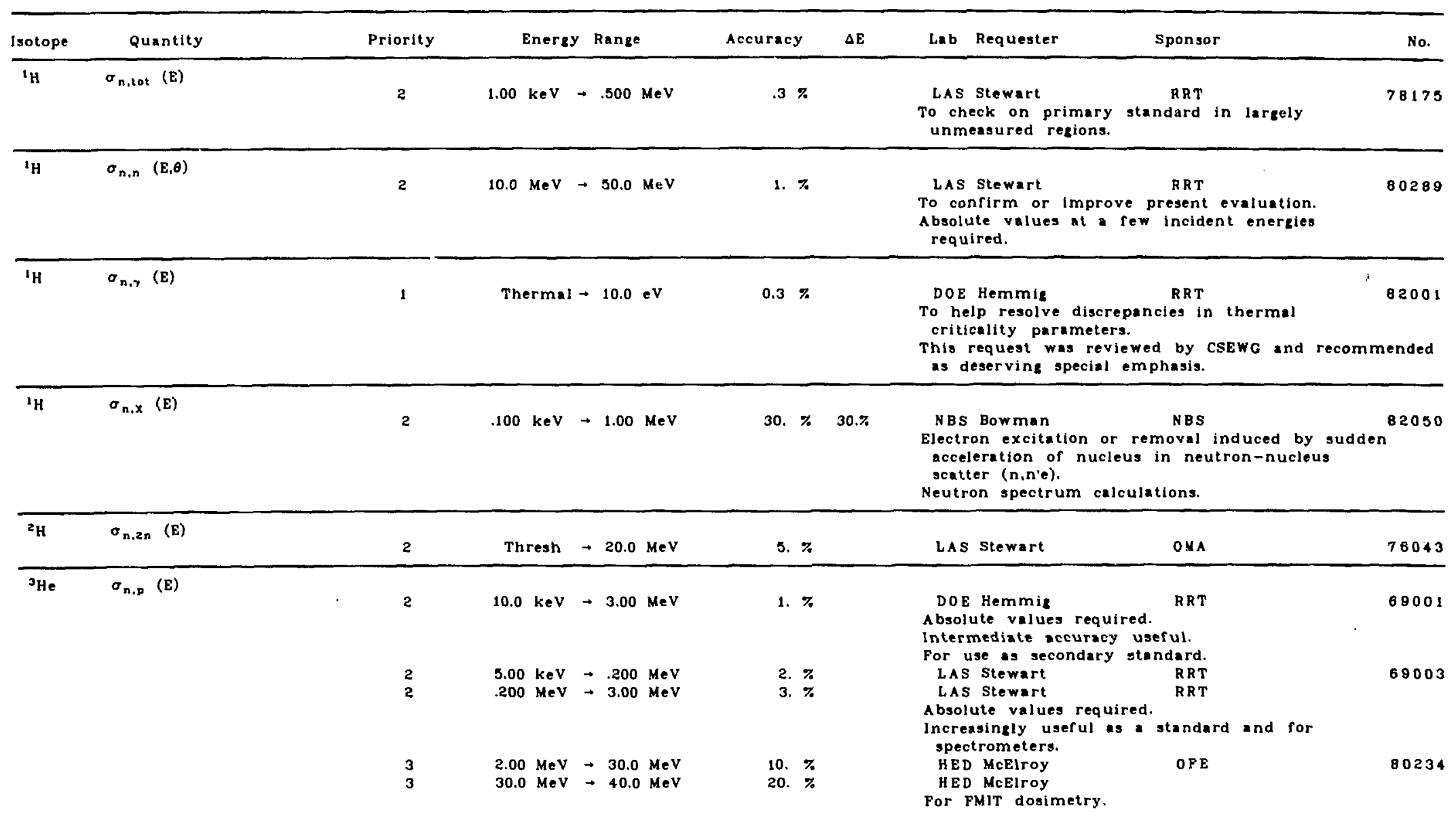


DOE-NDC Compilation of Requests for Nuclear Data 1982

\begin{tabular}{|c|c|c|c|c|c|c|c|c|c|c|}
\hline Isotope & Quantity & Priority & & Energ. & y Range & & Accuracy & $\Delta \mathbf{E}$ & Lab Requester & No. \\
\hline${ }^{3} \mathrm{He}$ & $\sigma_{n, n^{\prime} p}(E)$ & $\begin{array}{l}3 \\
3\end{array}$ & $\begin{array}{l}\text { Thr } \\
30.0\end{array}$ & $\begin{array}{l}\text { resh } \\
\text { MeV }\end{array}$ & $\begin{array}{l}\rightarrow \quad 30.0 \\
\rightarrow \quad 40.0\end{array}$ & $\begin{array}{l}\mathrm{MeV} \\
\mathrm{MeV}\end{array}$ & $\begin{array}{l}10 . \% \\
20 . \%\end{array}$ & & $\begin{array}{l}\text { HED MCEIroy } \\
\text { HED MCElroy } \\
\text { For FMIT dosimetry. }\end{array}$ & B0235 \\
\hline${ }^{3} \mathrm{He}$ & $\sigma_{n, z p}(E)$ & $\begin{array}{l}3 \\
3\end{array}$ & $\begin{array}{l}\text { Thr } \\
\mathbf{3 0 . 0}\end{array}$ & $\begin{array}{l}\text { resh } \\
\text { MeV }\end{array}$ & $\begin{array}{l}\rightarrow 30.0 \\
\rightarrow \quad 40.0\end{array}$ & $\begin{array}{l}\mathrm{MeV} \\
\mathrm{MeV}\end{array}$ & $\begin{array}{l}16 . \% \\
20 . \%\end{array}$ & & $\begin{array}{l}\text { HED MCElroy } \\
\text { HED MCEIroy } \\
\text { For FMIT dosimetry. }\end{array}$ & 80233 \\
\hline$n n t_{L i}$ & $\sigma_{a, n}(E)$ & 2 & .100 & $\mathrm{MeV}$ & $\rightarrow 6.50$ & $\mathrm{MeV}$ & 6. $\%$ & & $\begin{array}{l}\text { LAS Walton } \\
\text { Thick target yields required. } \\
\text { Relative error of } 3.0 \% \text { needed. } \\
\text { a energy resolution } 100 \mathrm{keV} \text {. }\end{array}$ & 78167 \\
\hline${ }^{6} \mathrm{Li}$ & $\sigma_{n, t}(E)$ & $\begin{array}{l}1 \\
1 \\
2\end{array}$ & $\begin{array}{l}1.00 \\
1.00 \\
.500\end{array}$ & $\begin{array}{l}\mathrm{keV} \\
\mathrm{keV} \\
\mathrm{keV}\end{array}$ & $\begin{array}{l}\rightarrow \quad 3.00 \\
\vec{*} 3.00 \\
+3.00 \\
+\quad 100 \\
\rightarrow \quad 10.0\end{array}$ & $\begin{array}{l}\mathrm{MeV} \\
\mathrm{MeV} \\
\mathrm{MeV} \\
\mathrm{MeV} \\
\mathrm{eV}\end{array}$ & 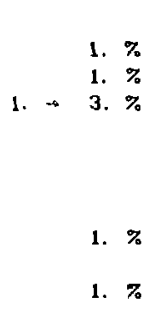 & & $\begin{array}{l}\text { ANL Smith } \\
\text { DOE Hemmig } \\
\text { LAS Hale } \\
\text { A RRT } \\
\text { A bsolute values required. } \\
\text { ART } \\
\text { Energy of } 3 \% \text { useful. } \\
\text { For use as standard. } \\
\text { LAS Hale } \\
\text { For use as standard below } 1 \text { MeV. } \\
\text { NBS Carlson } \\
\text { To study atomic binding and related effects. }\end{array}$ & $\begin{array}{l}72009 \\
80290\end{array}$ \\
\hline${ }^{\circ} \mathrm{LI}$ & $\sigma_{n, \ell}(E, \theta)$ & 2 & .500 & $\mathrm{keV}$ & $\Rightarrow 3.00$ & $\mathrm{MeV}$ & 5. 7 & & $\begin{array}{l}\text { LAS Hale } \\
\text { Absolute cross section as a function of angle. } \\
\text { Needed for use of } L i^{\circ}(n, \alpha) \text { as standard. }\end{array}$ & 80291 \\
\hline
\end{tabular}

STATUS: LAS Hale: REQ.80291. Work in progress al several places:

Drose (Vienna), Conde (Stockholm), lane (Ohio). 


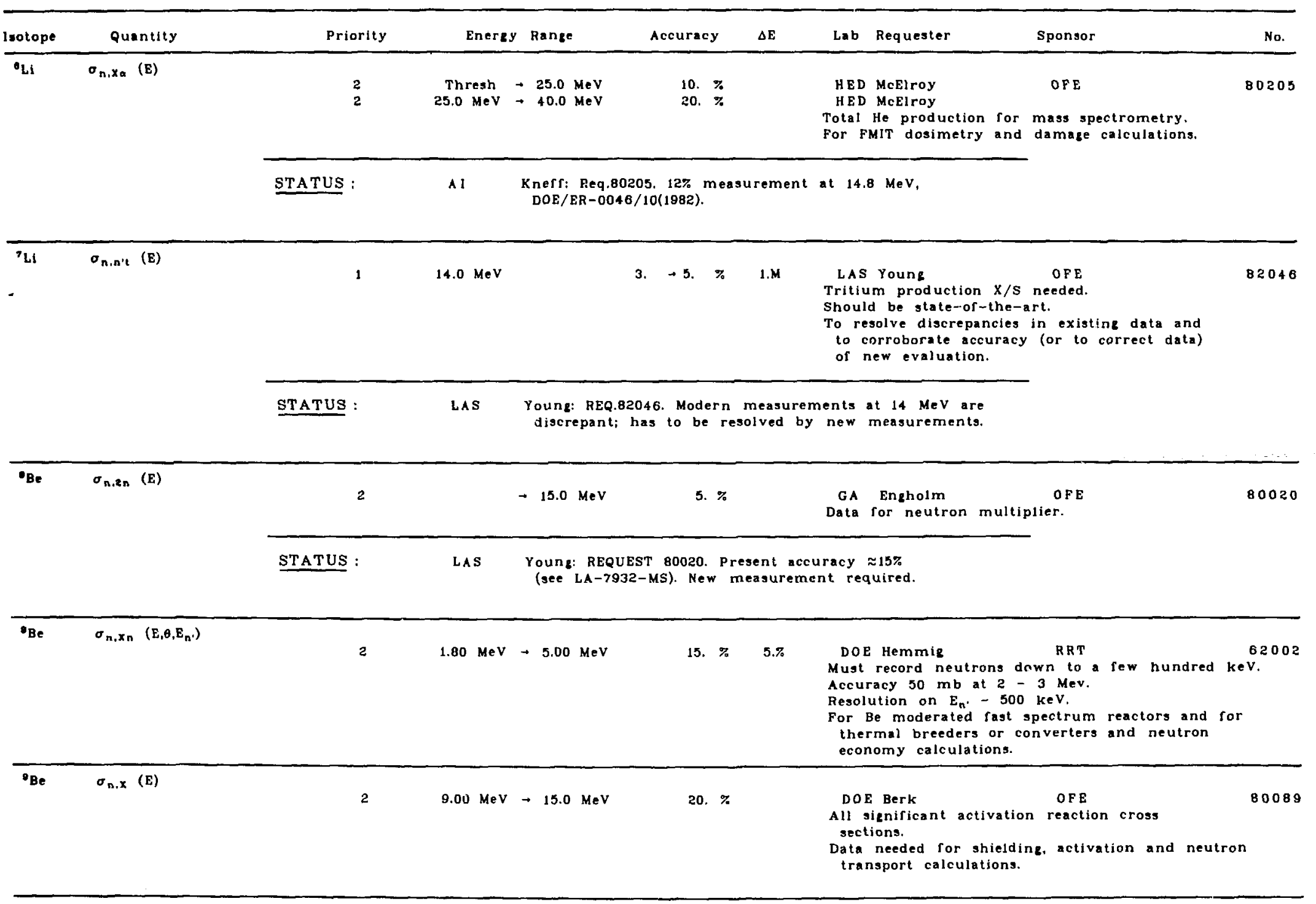


DOE-NDC Compilation of Requests for Nuclear Data 1982

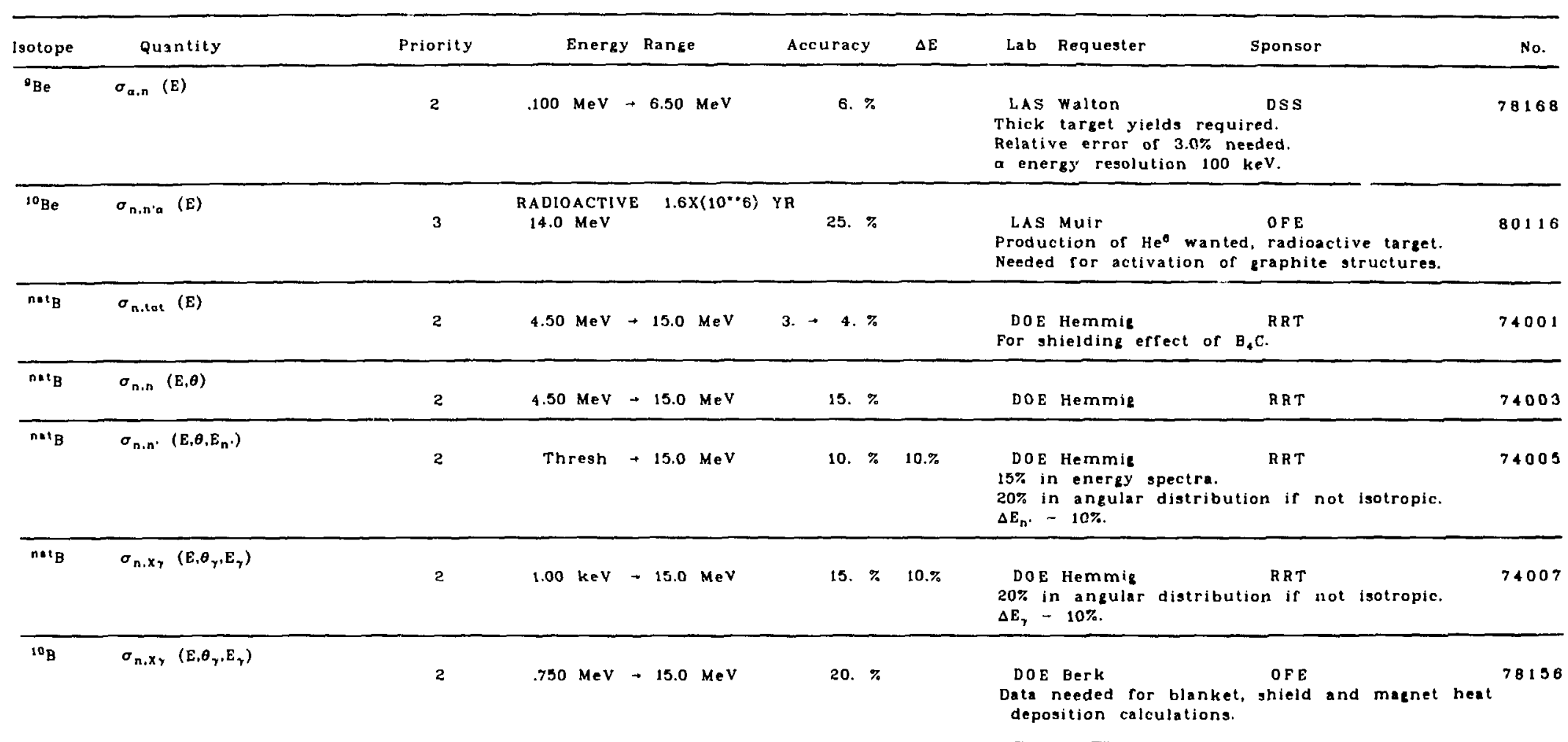

STATUS: LAS Young: REQ.78156. New evaluation needed.


DOE-NDC Compilation of Requests for Nuclear Data 1982

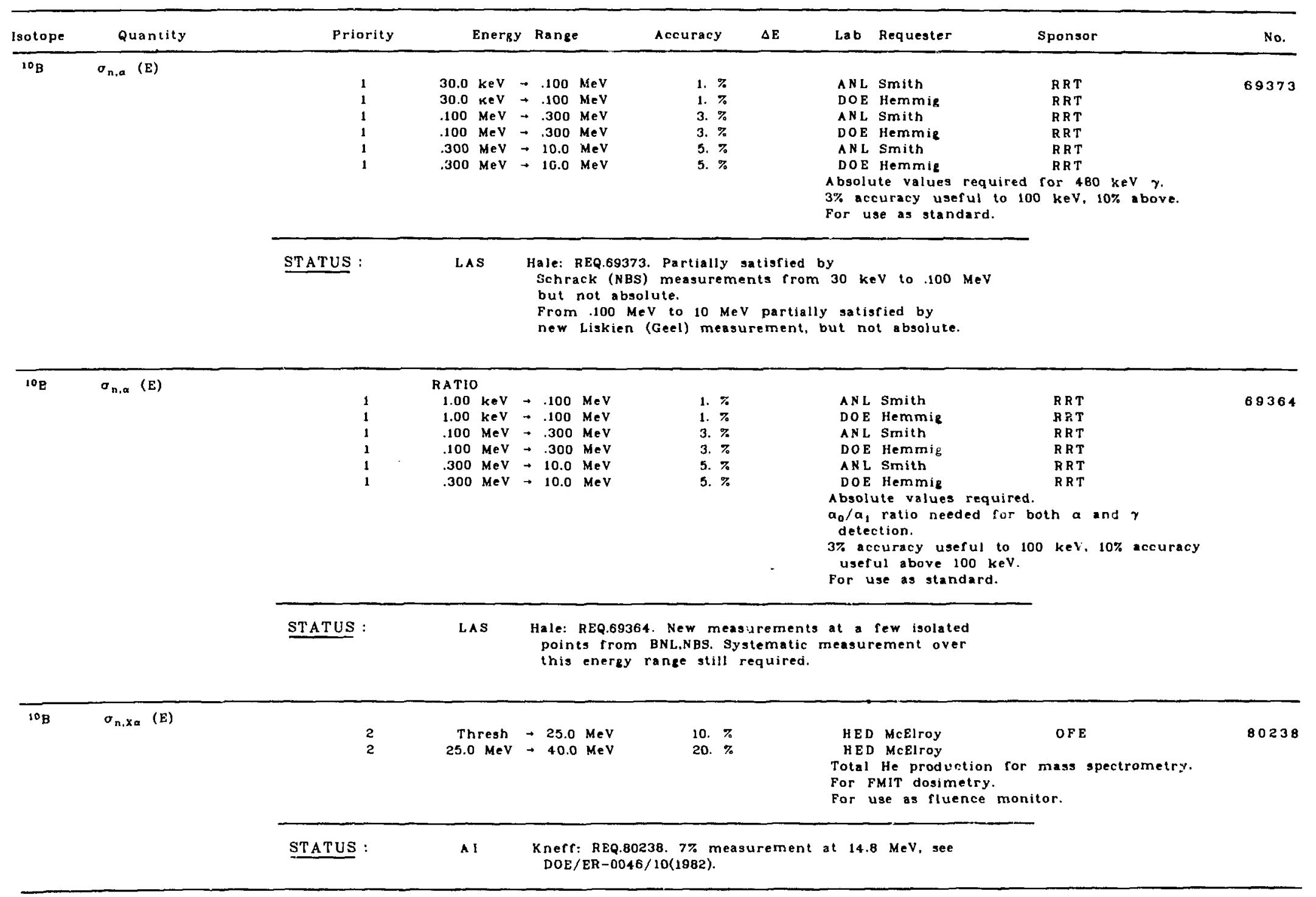


DOE-NDC Compilation of Requests for Nuclear Data 1982

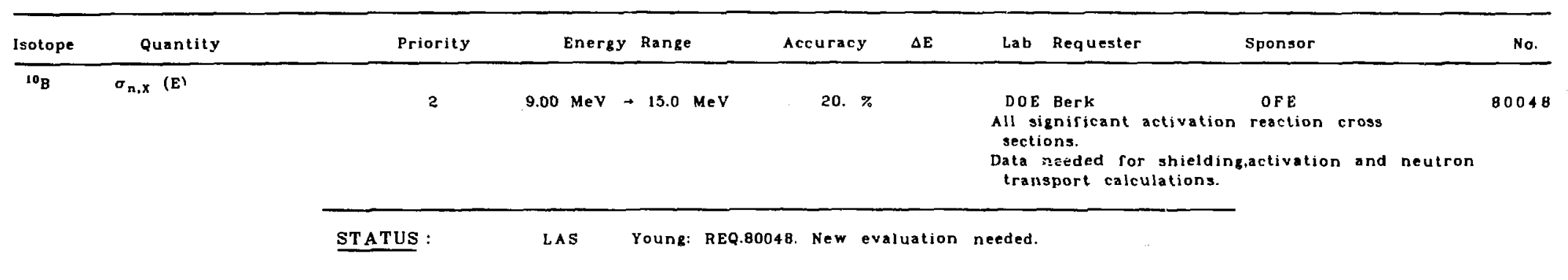

${ }^{\prime \prime B} \quad \sigma_{n, X \gamma}\left(E, \theta_{\gamma}, E_{\gamma}\right)$

$2.10 \mathrm{MeV} \rightarrow 15.0 \mathrm{MeV}$

20. $\%$

DOE Berk

OFE

78157

Dat needed for blanket, shield and masnet heat

deposition calculations.

STATUS : $\quad$ LAS Young: REQ.z8157, New evaluation needed.

\begin{tabular}{|c|c|c|c|c|c|c|c|c|}
\hline${ }^{11} B$ & $\sigma_{n, a}(E)$ & $\begin{array}{l}3 \\
3\end{array}$ & $\begin{array}{c}\text { Thresh } \\
25.0 \mathrm{MeV}\end{array}$ & $\begin{array}{l}\rightarrow \quad 25.0 \\
+\quad 40.0\end{array}$ & $\begin{array}{l}\mathrm{MeV} \\
\mathrm{MeV}\end{array}$ & $\begin{array}{l}10 . \% \\
20 . \%\end{array}$ & $\begin{array}{l}\text { HED MCElroy } \\
\text { HED MCElroy } \\
\text { FOF FMIT dosimetry and track recorder. }\end{array}$ & 80221 \\
\hline natc & $\sigma_{n, n^{\prime} \cdot}\left(E, \theta_{\gamma}\right)$ & $\mathbf{z}$ & $5.00 \mathrm{MeV}$ & $\rightarrow 20.0$ & $\mathrm{MeV}$ & 5. $\%$ & $\begin{array}{l}\text { ORL Fu } \\
\text { For } 4.43 \text { MeV Y's only. } \\
\text { Measure ror at least rour angles. }\end{array}$ & 74177 \\
\hline natc & $\sigma_{n, x_{n}}\left(E, \theta, E_{n}\right)$ & $\boldsymbol{2}$ & $9.00 \mathrm{MeV}$ & $\rightarrow 15.0$ & MeV & 10. $\%$ & $\begin{array}{l}\text { DOE Berk } \\
\text { For shielding and transport studies of next } \\
\text { generation } D \rightarrow T \text { reactor designs. }\end{array}$ & 78043 \\
\hline
\end{tabular}

STATUS : $\quad$ ORL Larson: REQ.78043. For secondary neutron spectra

from thick $C$ sample. See ORNL-TM-5B14 (1977) 
DOE-NDC Compilition of Requests for Nuclear Data 1982

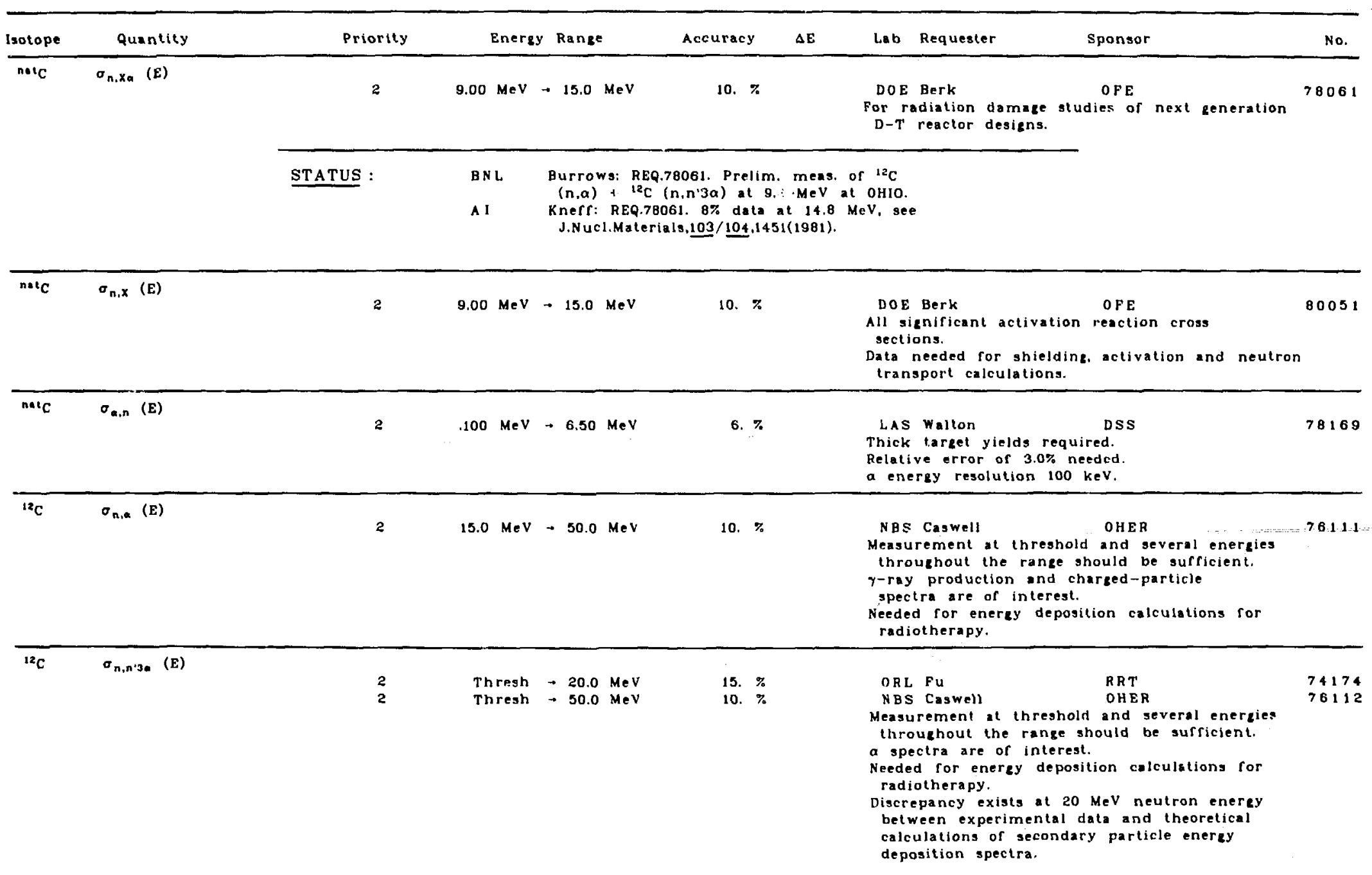


DOE-NDC Compilation of Requests for Nuclear Data 1982

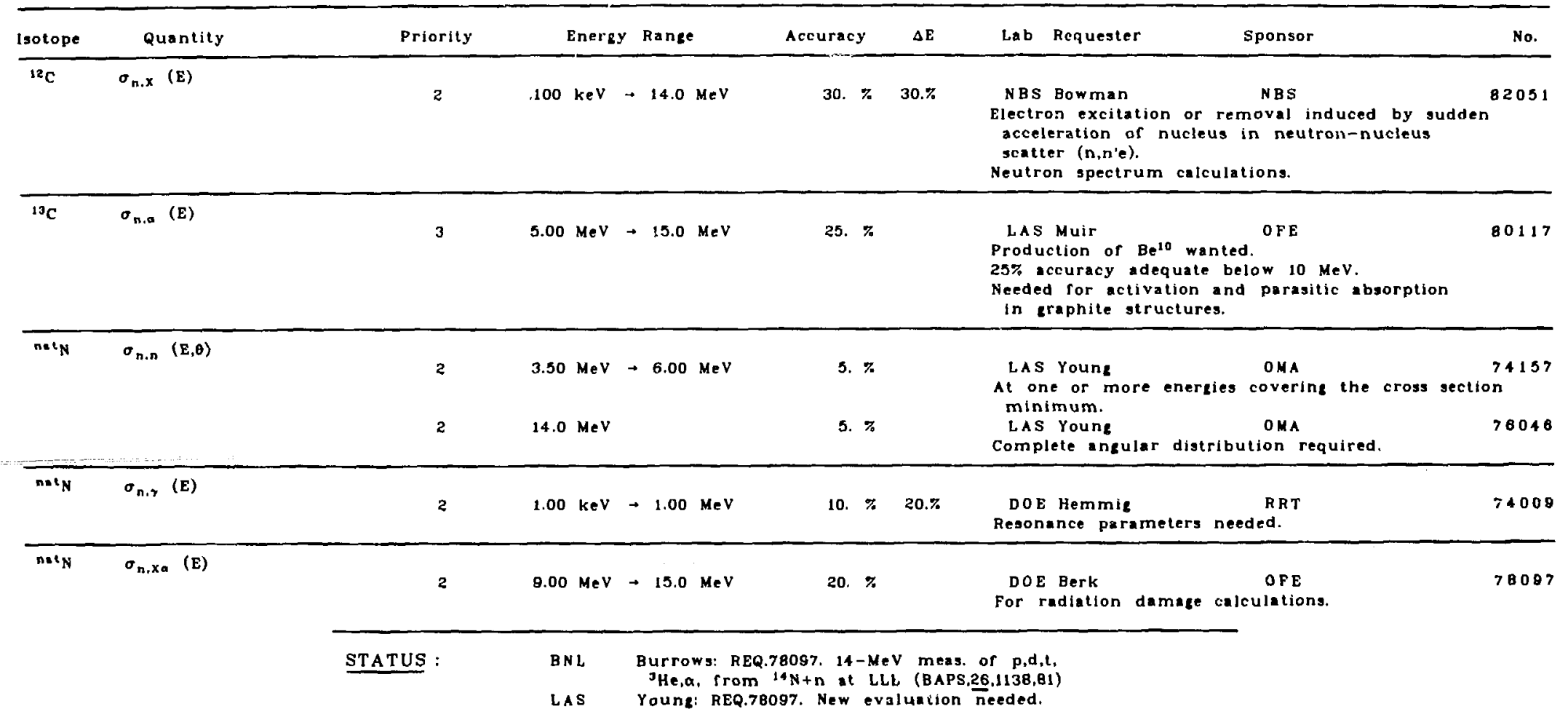




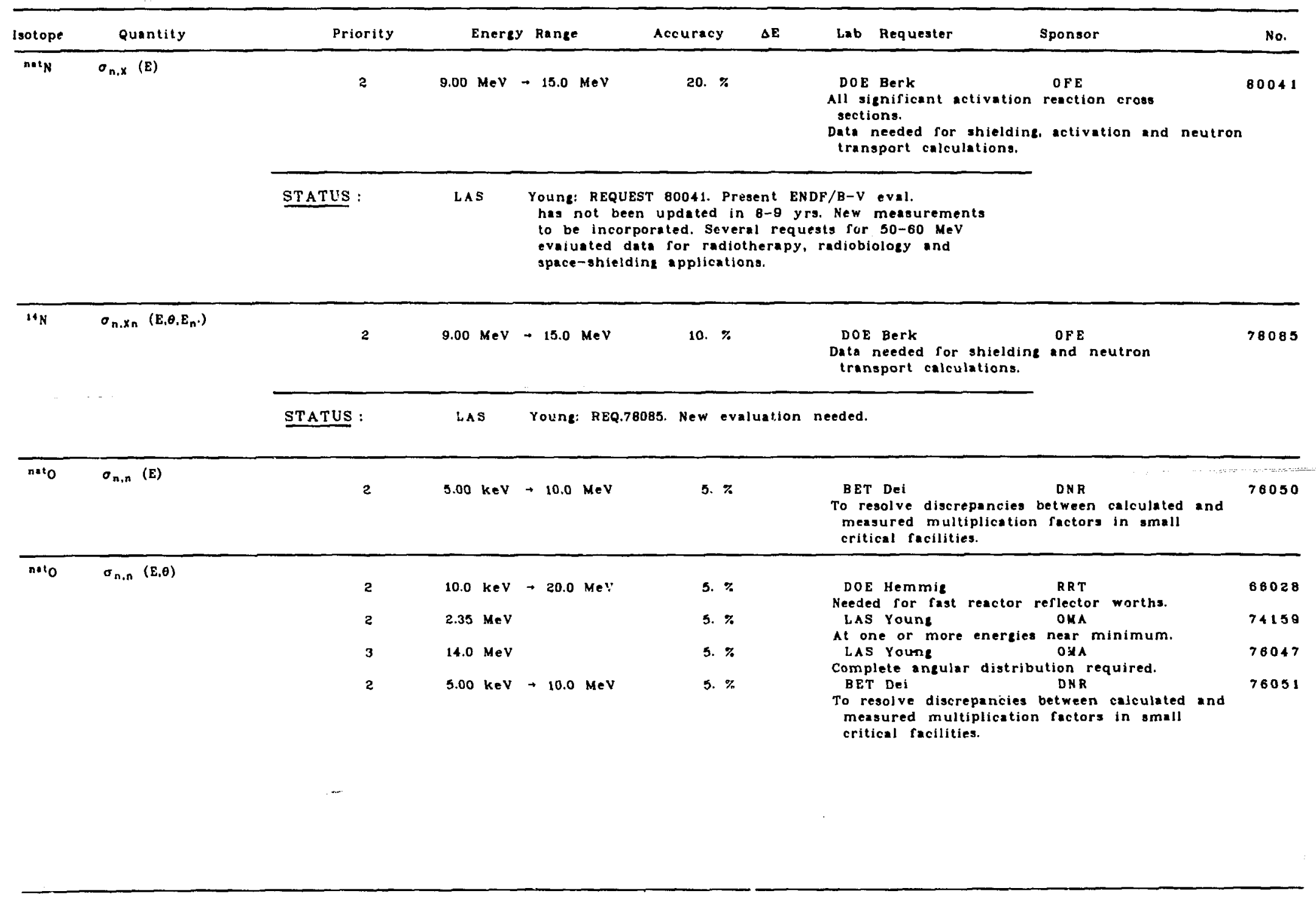


DOE-NDC Compilation of Requests for Nuclear Data 1982

\begin{tabular}{cccccccc}
\hline Isotope & Quantity & Priority & Enerzy Range & Accuracy & $\Delta E$ & Lab Requester & Sponsor \\
\hline nato & $\sigma_{n, X n}\left(E, \theta_{1} E_{n^{\prime}}\right)$ & 1 & $8.00 \mathrm{MeV} \rightarrow 15.0 \mathrm{MeV}$ & $20 . \%$ & $\begin{array}{c}\text { DoE Berk } \\
\text { Data needed for shielding. OPE } \\
\text { transport calculations. }\end{array}$
\end{tabular}

transport calculations.

STATUS: LAS Young: REQ.78089. New evaluation needed.

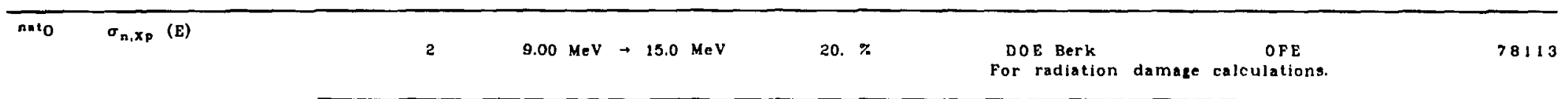

\begin{tabular}{|c|c|c|}
\hline STATUS: & $\begin{array}{l}\text { BNL } \\
\text { LAS }\end{array}$ & $\begin{array}{l}\text { Burrows: REQ.78113. 14-MeV meas. of p,d,t, } \\
{ }^{3} \mathrm{He}, \alpha_{1} \text { from }{ }^{10} 0+n \text { at LLL (BAPS,26,1138,81) } \\
\text { Young: REQ.78113. New evaluation needed. }\end{array}$ \\
\hline
\end{tabular}

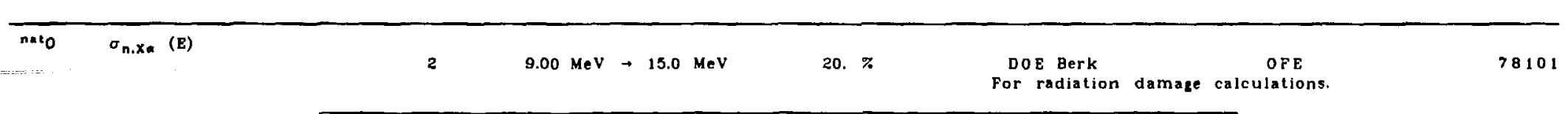

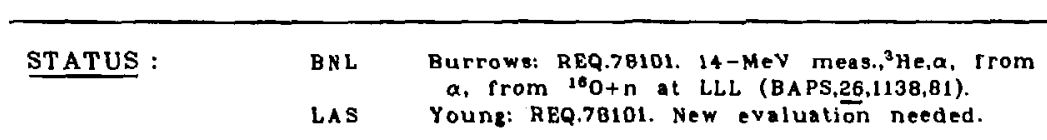

\begin{tabular}{|c|c|c|c|c|c|c|c|}
\hline \multirow[t]{2}{*}{ nato } & \multirow[t]{2}{*}{$\sigma_{n, x}(E)$} & 2 & $9.00 \mathrm{MeV}$ & $\rightarrow 15.0 \mathrm{MeV}$ & $20 . \%$ & $\begin{array}{l}\text { DOE Berk } \\
\text { All significant activation } \\
\text { sections. } \\
\text { Data needed for shielding } \\
\text { transport calculations. }\end{array}$ & \multirow[t]{2}{*}{80042} \\
\hline & & STATUS : & LAS & Yount: REQ.80042. & New evaluation & needed. & \\
\hline nalo & $\sigma_{a, n}(E)$ & 2 & $.100 \mathrm{MeV}$ & $\rightarrow \quad 6.50 \mathrm{MeV}$ & 6. $\%$ & $\begin{array}{l}\text { LAS Walton } \\
\text { Thick target yields required. } \\
\text { Relative error of } 3.0 \% \text { needed. } \\
\text { a energy resolution } 100 \mathrm{keV} \text {. }\end{array}$ & 78170 \\
\hline
\end{tabular}


DOE-NDC Compilation of Requests for Nuclear Data 1982

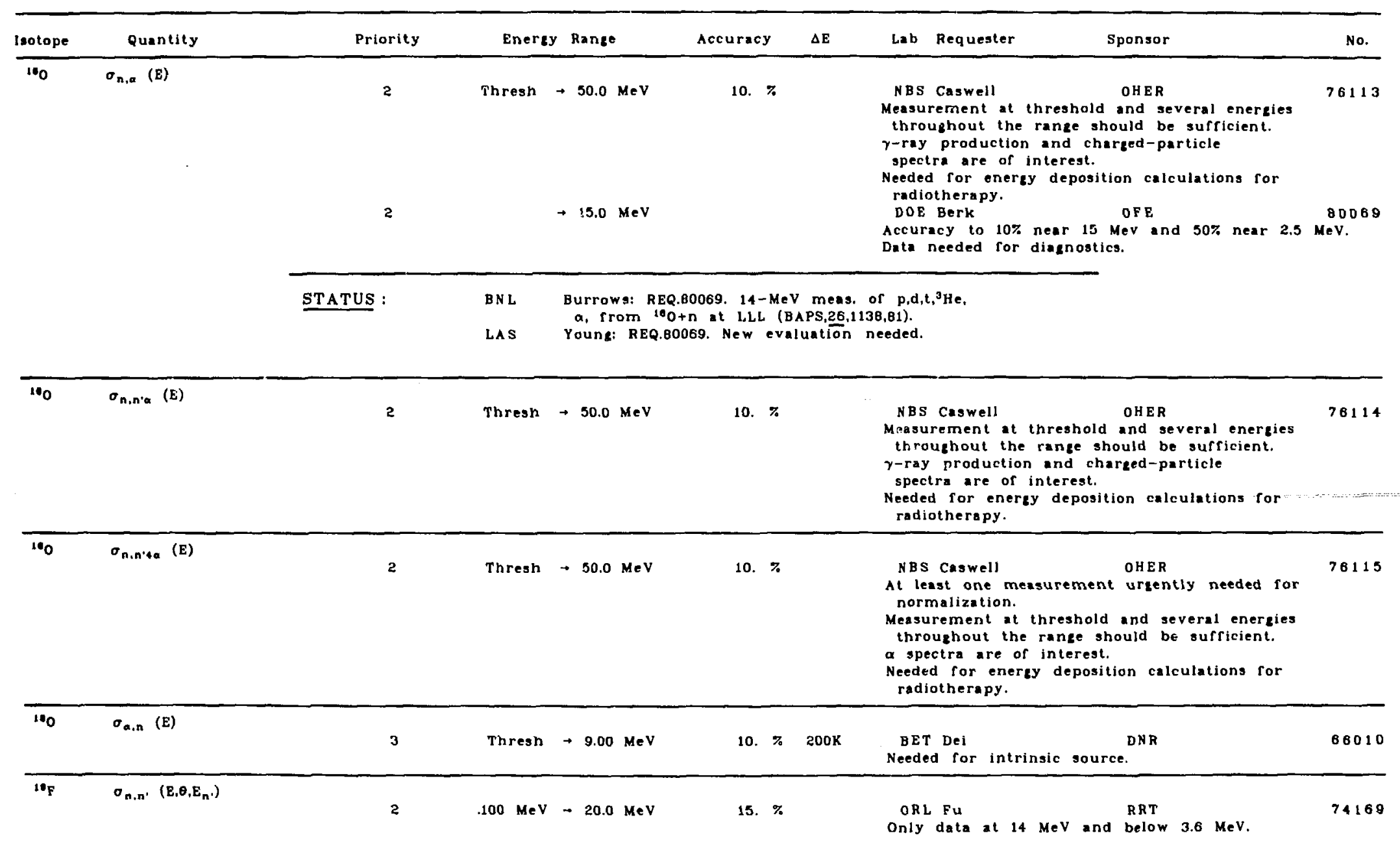


DOE-NDC Compilation of Requests for Nuclear Data 1982

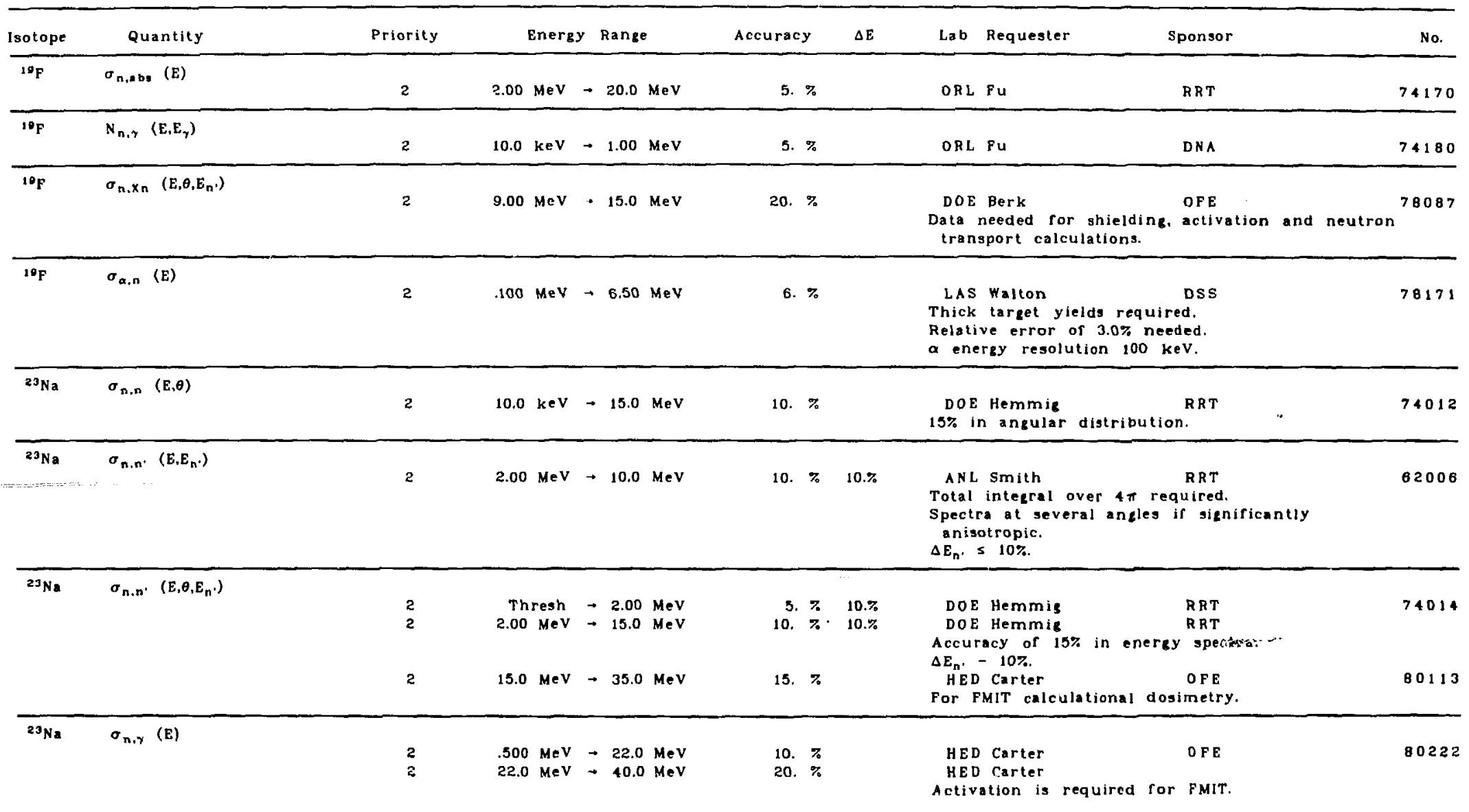




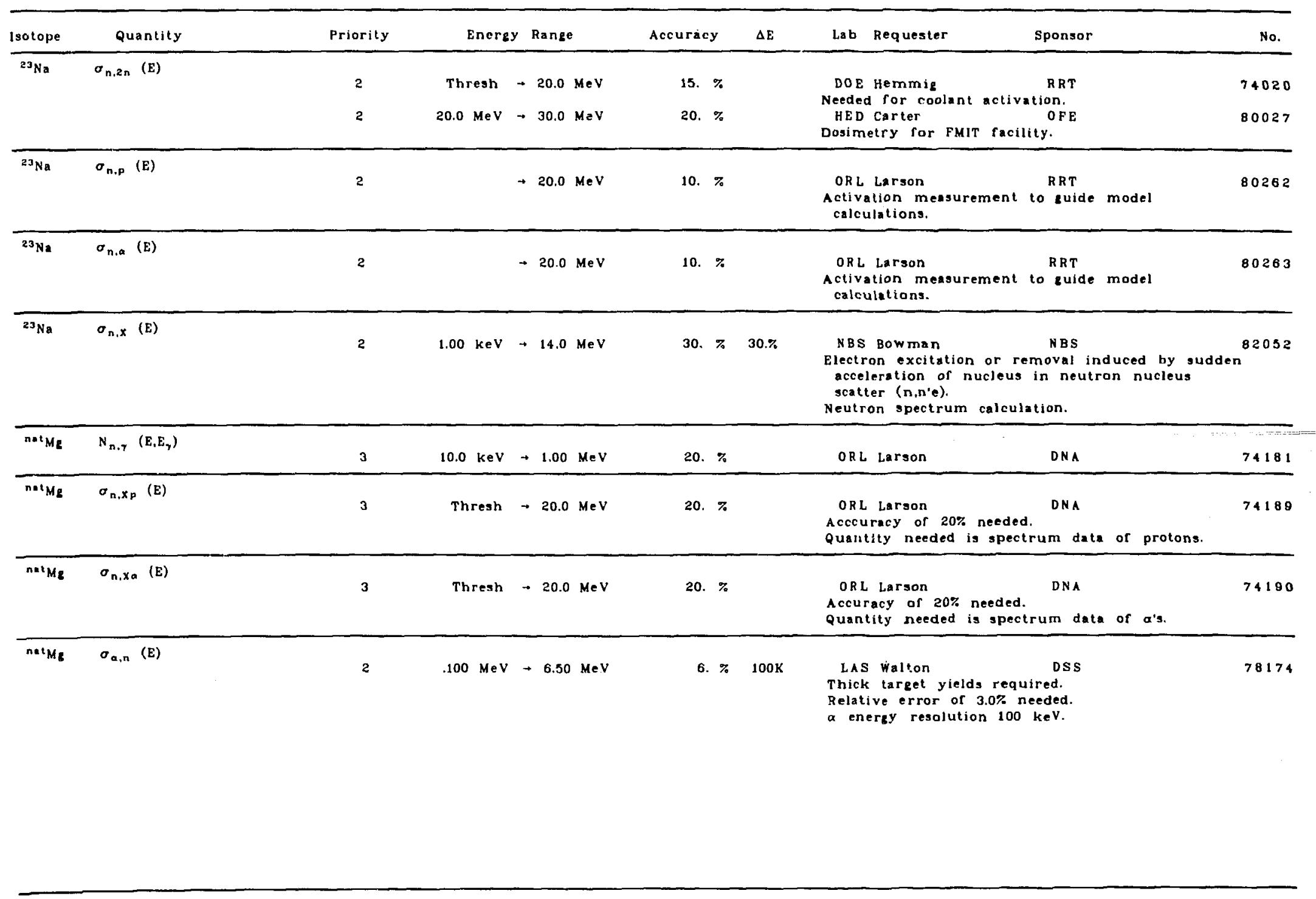


DOE-NDC Compilation of Requests for Nuclear Data 1982

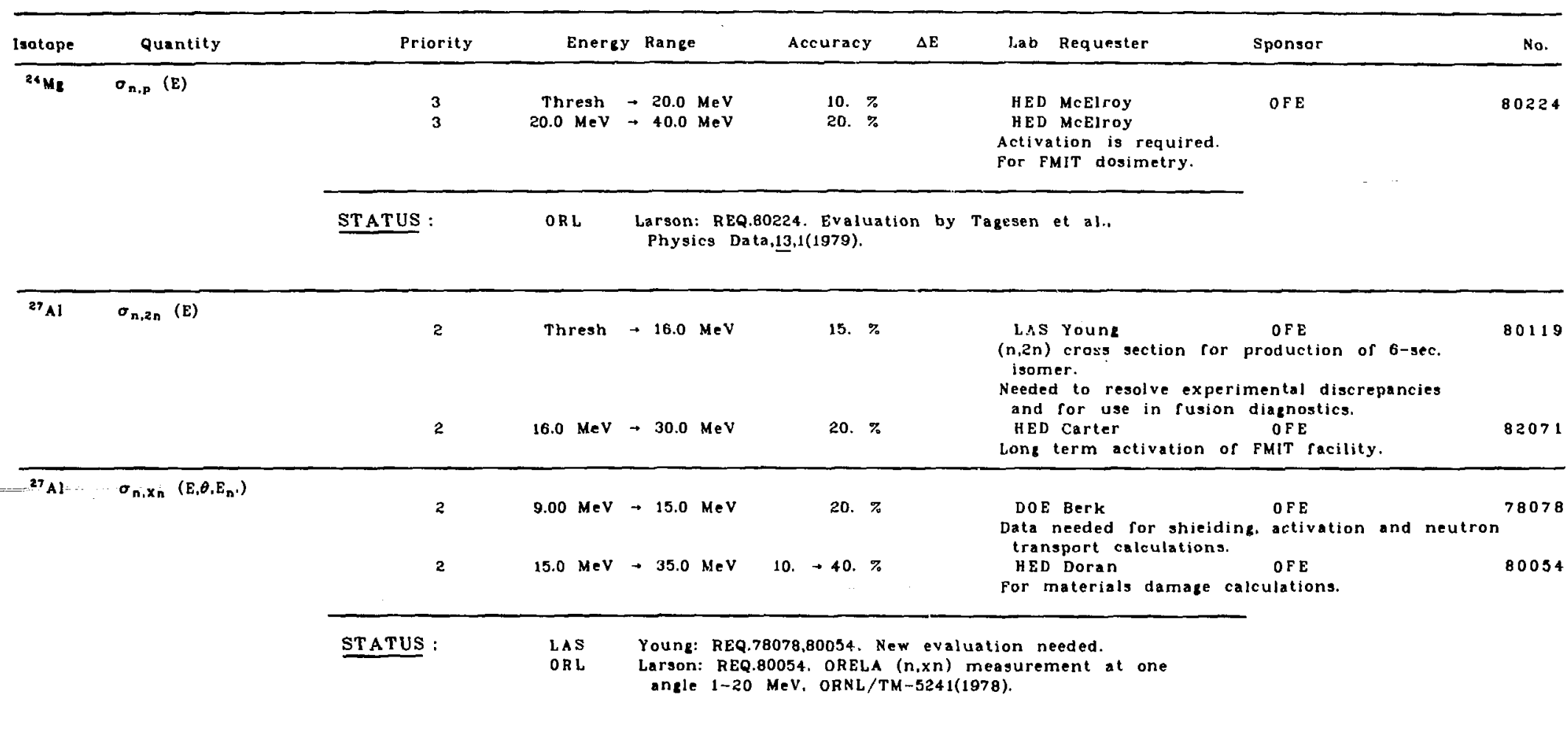

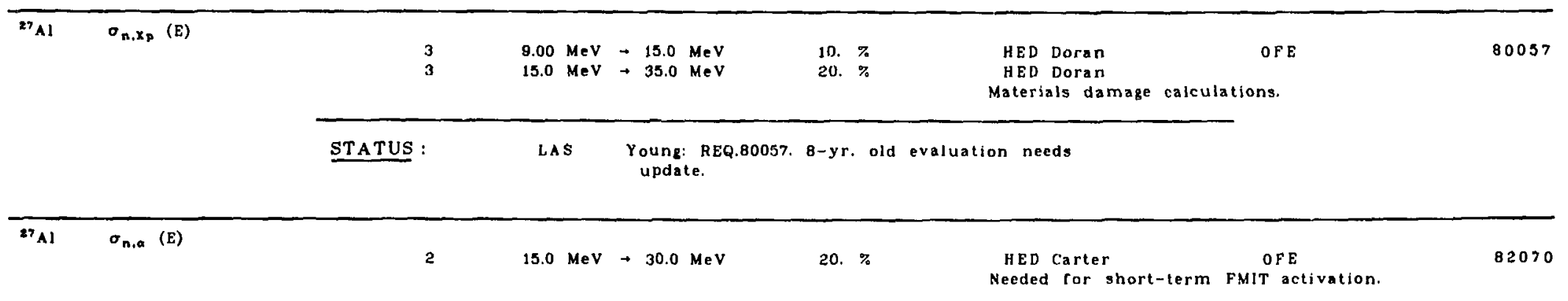


DOE-NDC Compilation of Requests for Nuclear Data 1982

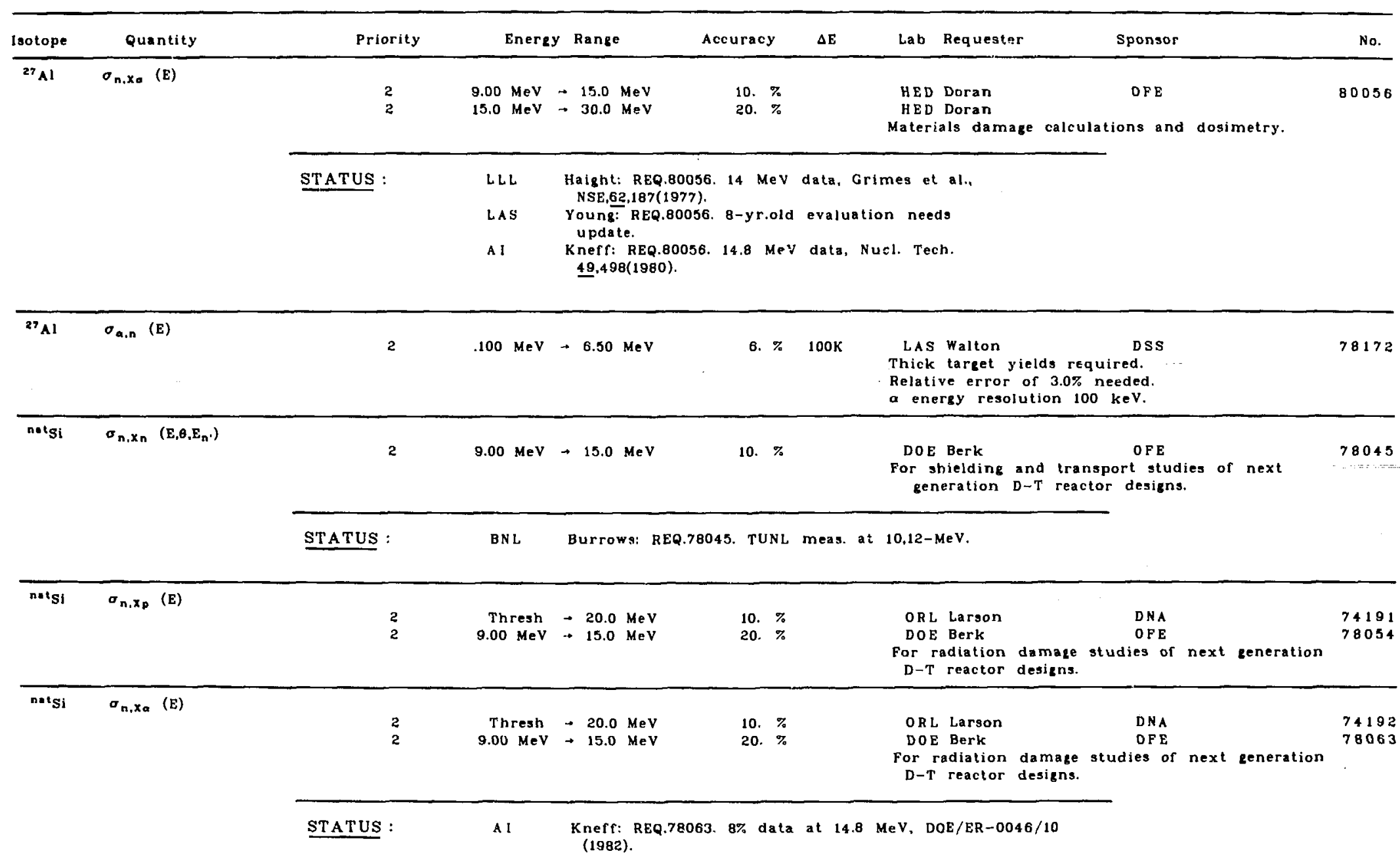


DOE-NDC Compilation of Requests for Nuclear Data 1982

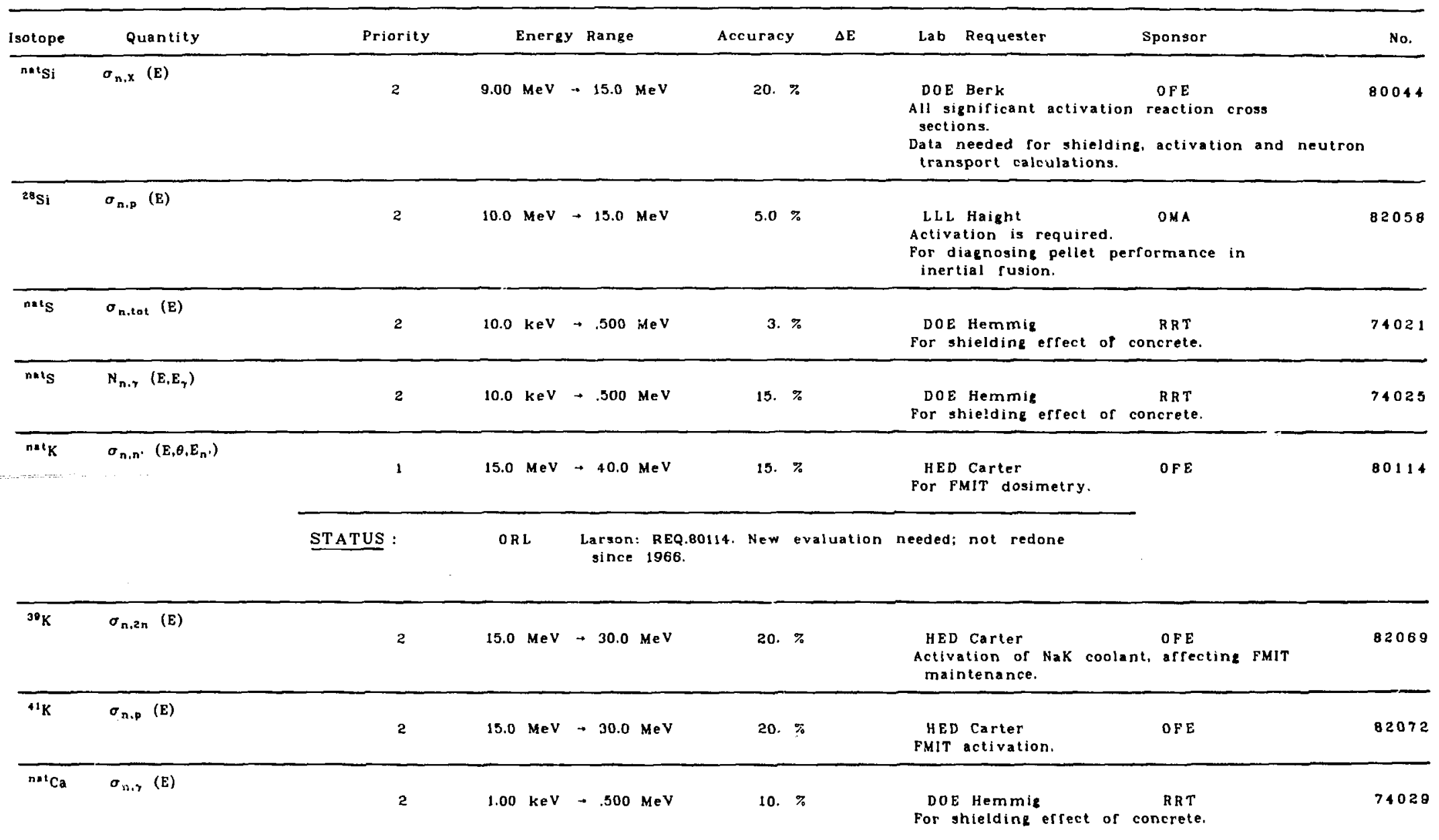


DOE-NDC Compilation of Requests for Nuclear Data 1982

\begin{tabular}{|c|c|c|c|c|c|c|c|c|c|c|}
\hline sotope & Quantity & Priority & & Enersy & y Range & & Aceuracy & $\Delta \mathrm{E}$ & Lab Requester & No. \\
\hline natca & $N_{n, \gamma}\left(E, E_{\gamma}\right)$ & 3 & 2.00 & keV & $\rightarrow 1.00$ & $\mathrm{MeV}$ & 5. $\%$ & & ORL Fu & 74183 \\
\hline$n=1 C a$ & $\sigma_{a, n}(E)$ & 2 & .100 & $\mathrm{MeV}$ & $\rightarrow 6.50$ & $\mathrm{MeV}$ & 6. $\%$ & $100 \mathrm{~K}$ & $\begin{array}{l}\text { LAS walton } \\
\text { Thick taret yields required. } \\
\text { Relative error of } 3.0 \% \text { needed. } \\
\text { a energy resolution } 100 \text { kev. }\end{array}$ & 78173 \\
\hline${ }^{15} \mathrm{Sc}$ & $\sigma_{n, \gamma}(E)$ & 2 & .100 & MeV & $\rightarrow \quad 18.0$ & $\mathrm{MeV}$ & 10. $\%$ & & $\begin{array}{l}\text { HED McEIroy } \\
\text { Activation is required. } \\
\text { For use as fluence monitor. }\end{array}$ & 69065 \\
\hline netTi & $\sigma_{\gamma, p}(E)$ & 2 & 10.0 & MeV & $\rightarrow \quad 20.0$ & $\mathrm{MeV}$ & 50.7 & & $\begin{array}{l}\text { DOE Berk } \\
\text { Reaction used to identify runaway electrons that } \\
\text { hit PDX limiters. }\end{array}$ & 80072 \\
\hline \multirow[t]{2}{*}{ nat Ti } & $\sigma_{n, \times n} \quad\left(E, \theta, E_{n}\right)$ & 2 & 15.0 & Mev & $\rightarrow 35.0$ & $\mathrm{MeV}$ & $10 .-40 \%$ & & $\begin{array}{l}\text { HED Doran } \\
\text { Accuracy to be determined from sensitivity } \\
\text { studies. } \\
\text { For material damage calculations. }\end{array}$ & 78039 \\
\hline & & STATUS : & BN 1 & 1. & $\begin{array}{l}\text { Burrow } \\
\text { a-em }\end{array}$ & $\begin{array}{l}\text { ws: REQ } \\
\text { nission }\end{array}$ & $\begin{array}{l}.78039 \text {. CORNL- } \\
\text { spectra meas. }\end{array}$ & $\begin{array}{c}-5563) \\
\text { up to }\end{array}$ & $\begin{array}{l}\text { Neutron and } \\
20-M e v \text {. }\end{array}$ & \\
\hline natTi & $\sigma_{n, x_{p}}(E)$ & $\begin{array}{l}2 \\
2\end{array}$ & $\begin{array}{l}9.00 \\
15.0\end{array}$ & $\begin{array}{l}\mathrm{MeV} \\
\mathrm{MeV}\end{array}$ & $\begin{array}{l}\Rightarrow \quad 15.0 \\
\Rightarrow \quad 30.0\end{array}$ & $\begin{array}{l}\mathrm{MeV} \\
\mathrm{MeV}\end{array}$ & $\begin{array}{l}\text { 20. \% } \\
\text { 20. \% }\end{array}$ & & $\begin{array}{l}\text { HED Doran } \\
\text { HED Doran } \\
\text { For material damage calculations. }\end{array}$ & 78027 \\
\hline \multirow[t]{2}{*}{$n=t T i$} & $\sigma_{n, x a}(E)$ & $\begin{array}{l}2 \\
2\end{array}$ & $\begin{array}{l}9.00 \\
15.0\end{array}$ & $\begin{array}{l}\mathrm{MeV} \\
\mathrm{MeV}\end{array}$ & $\begin{array}{l}\rightarrow \quad 15.0 \\
\rightarrow \quad 30.0\end{array}$ & $\begin{array}{l}\mathrm{MeV} \\
\mathrm{MeV}\end{array}$ & $\begin{array}{l}20 . \% \\
20 . \%\end{array}$ & & $\begin{array}{l}\text { HED Doran } \\
\text { HED Doran } \\
\text { For material damaze calculations and dosimetry. }\end{array}$ & 78212 \\
\hline & & STATUS: & AI & & $\begin{array}{r}\text { Kneff: } \\
\text { J.Nue }\end{array}$ & $\begin{array}{l}\text { : REQ.78 } \\
\text { cl.Materi }\end{array}$ & $\begin{array}{l}\text { 82!2. } 87 \text { data } \\
\text { ials,103/104,145 }\end{array}$ & $\begin{array}{l}\text { at } 14.8 \\
51(1981) \text {. }\end{array}$ & MeV, see & \\
\hline
\end{tabular}


DOE-NDC Compilation of Requests for Nuclear Data 1982

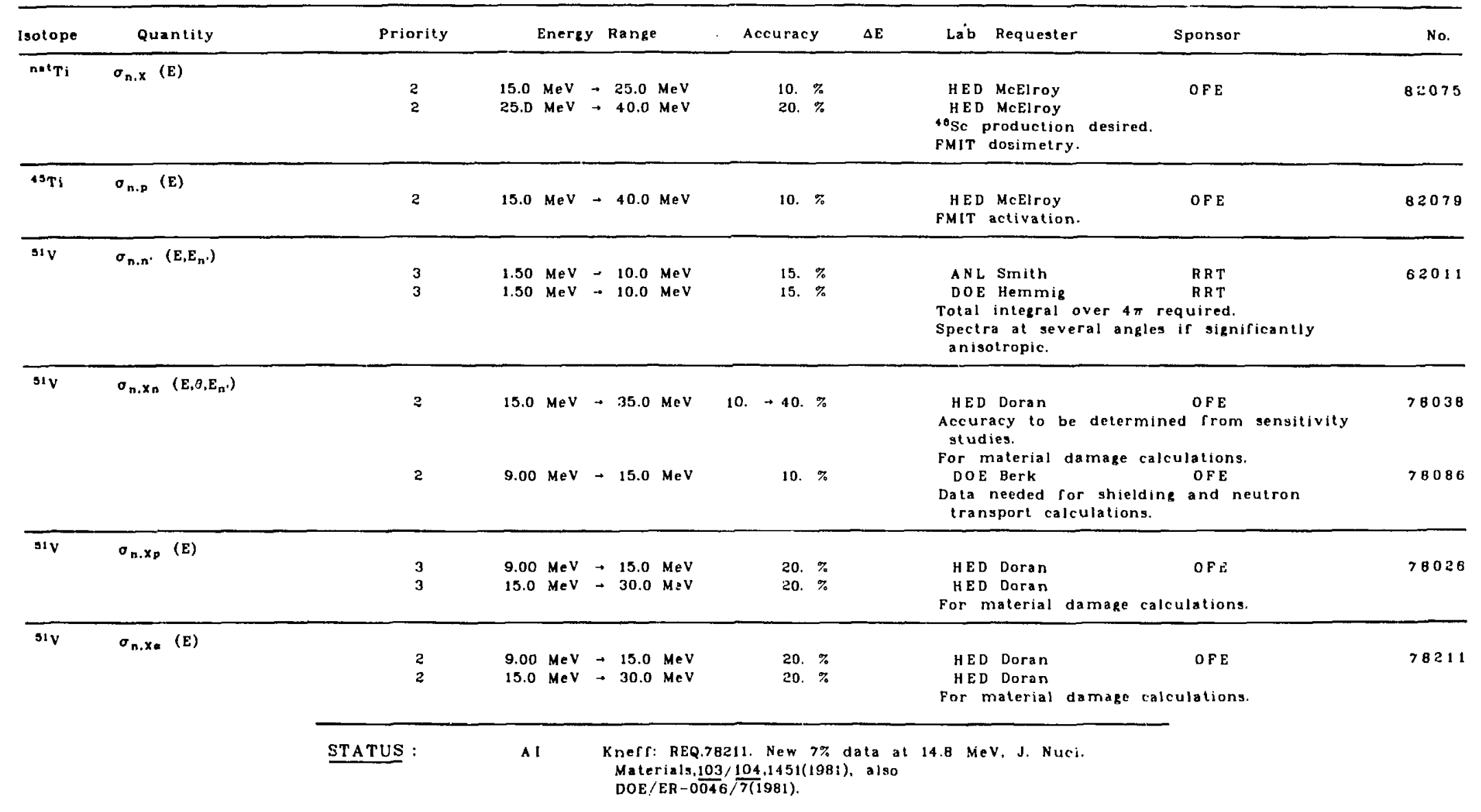


DOE-NDC Compilation of Requests for Nuclear Data 1982

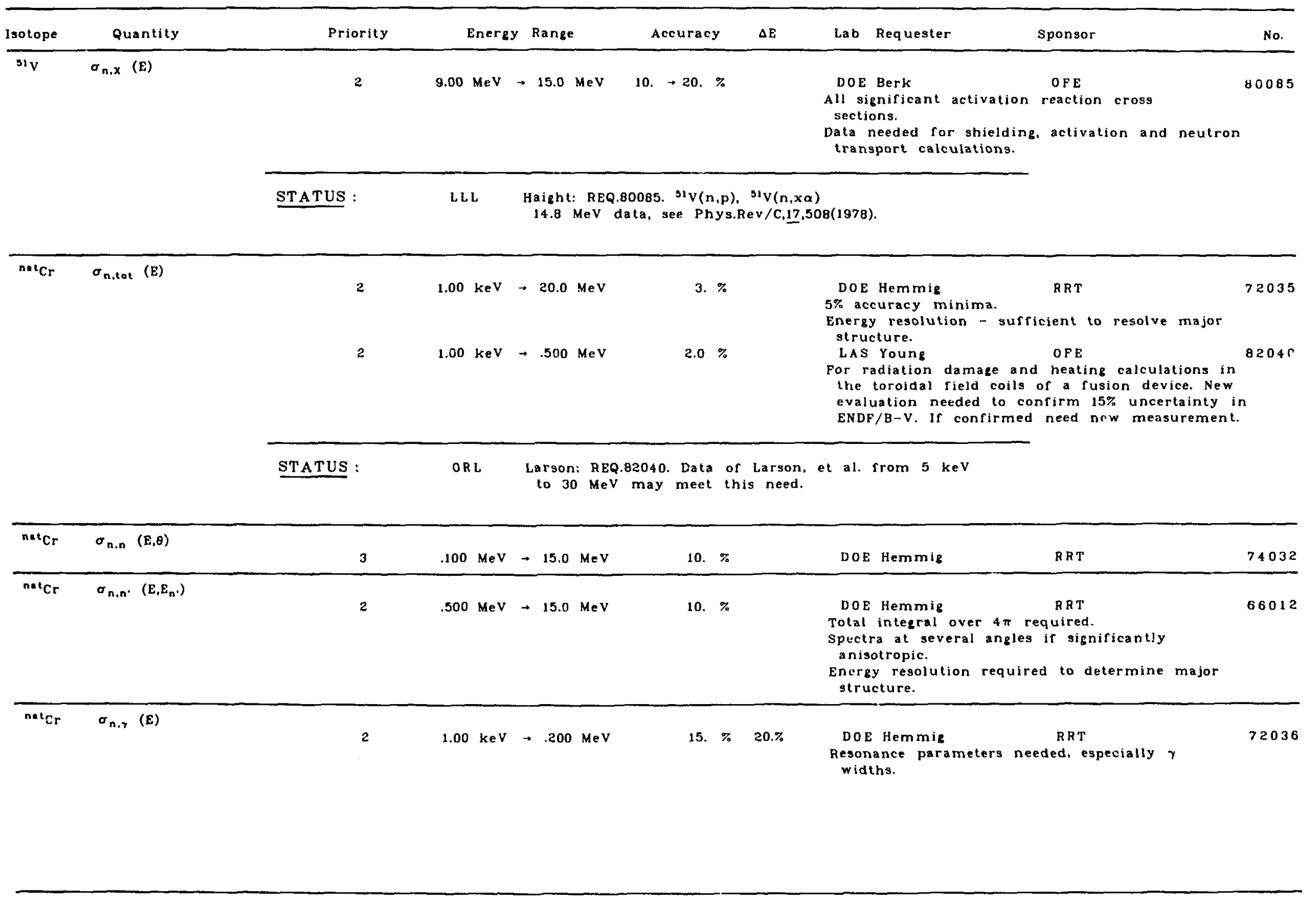


DOE-NDC Compilation of Requests for Nuclear Data 1982

\begin{tabular}{|c|c|c|c|c|c|c|c|c|}
\hline Isotope & Quantity & Priority & Enerey & y Range & Accuracy & $\Delta \mathbf{E}$ & Lab Requester & No. \\
\hline${ }^{50} \mathrm{Cr}$ & $\sigma_{n, \gamma}(E)$ & 2 & $25.3 \mathrm{mv}$ & $\rightarrow .300 \mathrm{MeV}$ & 10. $\%$ & & $\begin{array}{l}\text { BNL Prince } \\
\text { Activation rile. }\end{array}$ & 80124 \\
\hline${ }^{50} \mathrm{Cr}$ & $\sigma_{n .2 n}(E)$ & 2 & $14.0 \mathrm{MeV}$ & $\rightarrow 20.0 \mathrm{MeV}$ & $20 . \%$ & & $\begin{array}{l}\text { BNL Prince } \\
\text { Activation rile. }\end{array}$ & 80123 \\
\hline${ }^{32} \mathrm{Cr}$ & $\sigma_{n, 2 n}(E)$ & 2 & $\begin{array}{l}14.0 \mathrm{MeV}- \\
15.0 \mathrm{MeV}\end{array}$ & $\begin{array}{l}+\quad 20.0 \mathrm{MeV} \\
+\quad 35.0 \mathrm{MeV}\end{array}$ & $\begin{array}{l}20 . \% \\
20 . \%\end{array}$ & & $\begin{array}{l}\text { BNL Prince } \\
\text { Activation rile. } \\
\text { HED Carter } \\
\text { FMIT activation. }\end{array}$ & $\begin{array}{l}80122 \\
82080\end{array}$ \\
\hline${ }^{32} \mathrm{Cr}$ & $\sigma_{n, p}(E)$ & 2 & $\begin{array}{l}7.00 \mathrm{MeV}- \\
15.0 \mathrm{MeV}\end{array}$ & $\begin{array}{l}\rightarrow \quad 18.0 \mathrm{MeV} \\
\rightarrow \quad 35.0 \mathrm{MeV}\end{array}$ & $\begin{array}{l}25 . \% \\
20 . \%\end{array}$ & & $\begin{array}{lr}\text { BNL Prince } & \text { RRT } \\
\text { Hydrogen production evaluation. } \\
\text { HED Carter } & \text { OFE } \\
\text { FMIT activation. } & \end{array}$ & $\begin{array}{l}80126 \\
82084\end{array}$ \\
\hline${ }^{53} \mathrm{Cr}$ & $\sigma_{n, 3 n}(E)$ & 2 & 15.0 MeV - & $\rightarrow \quad 35.0 \mathrm{MeV}$ & 20. $\%$ & & $\begin{array}{l}\text { HED Carter } \\
\text { FMIT activation. }\end{array}$ & 82081 \\
\hline${ }^{54} \mathrm{Cr}$ & $\sigma_{n, 4 n}(E)$ & 2 & 15.0 $\mathrm{MeV}$ & $\Rightarrow 35.0 \mathrm{MeV}$ & $20 . \%$ & & $\begin{array}{l}\text { HED Carter } \\
\text { FMIT activation. }\end{array}$ & 82083 \\
\hline natMn & $\sigma_{n, X}(E)$ & 2 & $2.50 \mathrm{eV}$ & $\rightarrow 15.0 \mathrm{MeV}$ & $20 . \%$ & & $\begin{array}{l}\text { GA Engholm } \\
\text { Activation cross sectior. } \\
\text { Fusion reactor shutdown dose rates. }\end{array}$ & 80101 \\
\hline${ }^{95} \mathrm{Mn}$ & $\sigma_{n, 101}(E)$ & 2 & & & 4. $\%$ & & $\begin{array}{l}\text { ORL Fu } \\
\text { Need values in Fe windows. }\end{array}$ & 74195 \\
\hline${ }^{59} \mathrm{Mn}$ & $\sigma_{n, 2 n}(E)$ & 2 & $20.0 \mathrm{MeV}$ & $\rightarrow 30.0 \mathrm{MeV}$ & 10. $-20 . \%$ & & $\begin{array}{l}\text { HED McElroy } \\
\text { Dosimetry for FMIT facility. }\end{array}$ & 80022 \\
\hline
\end{tabular}


DOE-NDC Compilation of Requests for Nuclear Data 1982

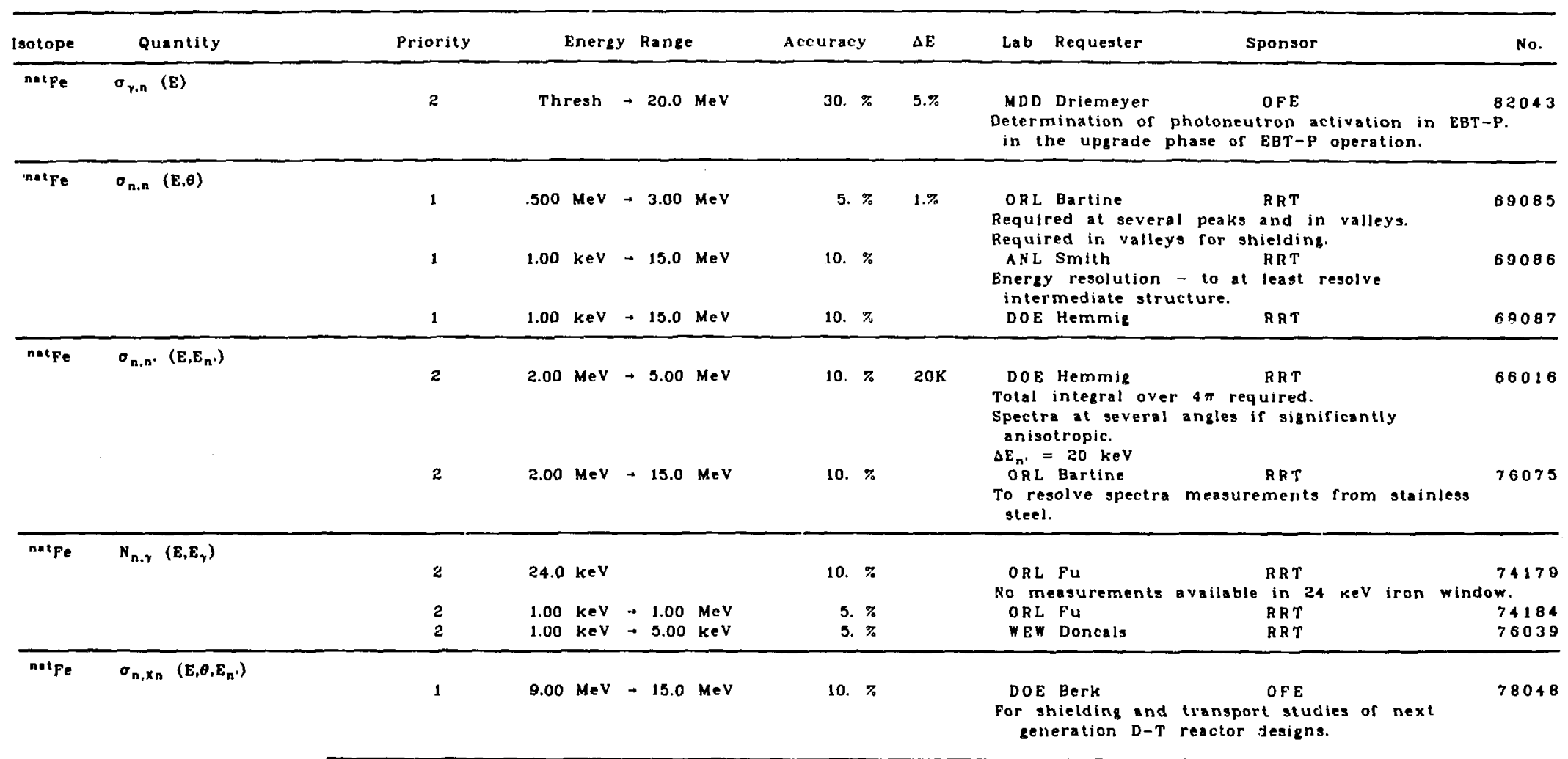

STATUS: $\quad$ BNL Burrows: REQ.78048. 2-30 MeV neutron and

$\alpha$-emission spectra under analysis at ORNL.

TUNL meas. at 10-12 MeV. 
DOE-NDC Compilation of Requests for Nuclear Data 1982

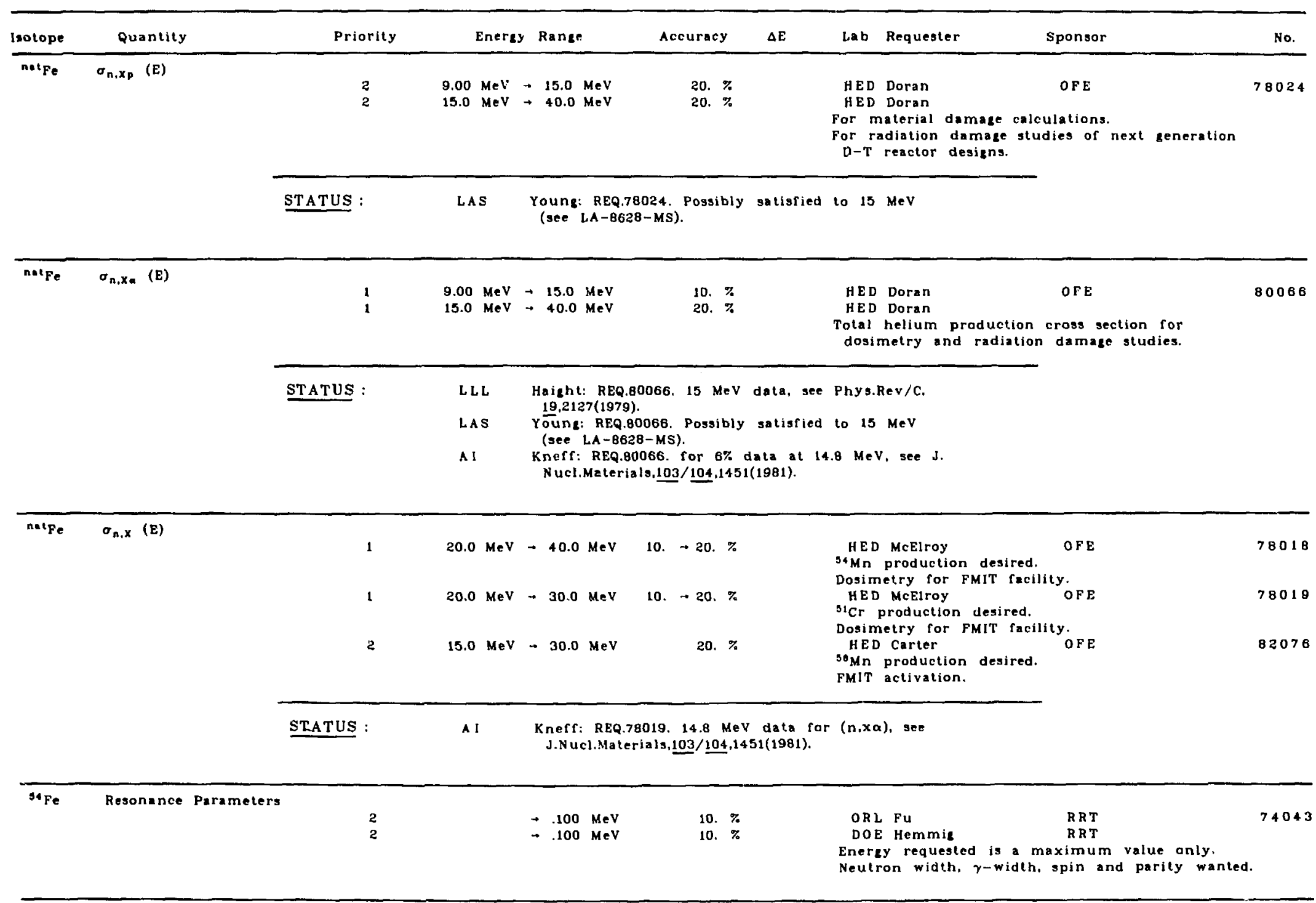


DOE-NDC Compilation of Requests for Nuclear Data 1982

\begin{tabular}{|c|c|c|c|c|c|c|c|c|c|}
\hline Isotope & Quantity & Priority & & Energy & y Range & & Accuracy & $\Delta \mathbf{E}$ & Lab Requester \\
\hline${ }^{50} \mathrm{Fe}$ & $\sigma_{n, \gamma}(E)$ & 1 & 10.0 & keV & $\rightarrow \quad 1.00$ & MeV & $10 . \rightarrow 15 \%$ & & $\begin{array}{l}\text { DOE Hemmie } \\
\text { Energy averaged accuracy to } 10-15 \% \text {. } \\
\text { This request was reviewed by CSEWG and recommended } \\
\text { as deserving special emphasis. }\end{array}$ \\
\hline${ }^{90} \mathrm{Fe}$ & $\sigma_{n, x}(E)$ & 2 & 1.00 & keV & $\rightarrow 14.0$ & $\mathrm{MeV}$ & 30. \% & $30 . \%$ & $\begin{array}{l}\text { NBS Bowman } \\
\text { Electron excitation or removal induced by sudden } \\
\text { acceleration or nucleus in neutron-nucleus } \\
\text { scatter (n,n'e). } \\
\text { Neutron spectrum calculations. }\end{array}$ \\
\hline${ }^{58} \mathrm{Fe}$ & Resonance Parameters & $\begin{array}{l}1 \\
1 \\
1\end{array}$ & & & $\begin{array}{l}\rightarrow \quad 400 \\
\rightarrow \quad 400 \\
\rightarrow \quad 400\end{array}$ & $\begin{array}{l}\mathrm{MeV} \\
\mathrm{MeV} \\
\mathrm{MeV}\end{array}$ & $\begin{array}{l}10 . \% \\
10 . \% \\
10 . \%\end{array}$ & & $\begin{array}{lc}\text { ORL Fu } & \text { RRT } \\
\text { DOE Hemmie } & \text { RRT } \\
\text { ANL Smith } & \text { RRT } \\
\text { Energy requested is a maximum value only. } \\
\text { Neutron width, y-width, spin and parity wanted, }\end{array}$ \\
\hline${ }^{57} \mathrm{Fe}$ & $\sigma_{n, n^{\prime}}(E)$ & 1 & & iresh & $\rightarrow 10.0$ & $\mathrm{MeV}$ & 20.7 & & $\begin{array}{l}\text { DOE Hemmie } \\
\text { Total inelastic scattering cross section needed. } \\
\text { This request was revitwed by CSEwC ind recommended } \\
\text { as deservine special emphasis. }\end{array}$ \\
\hline${ }^{38} \mathrm{Co}$ & $\sigma_{n, y}(E)$ & 2 & RADIC & OACTIV & VE $\quad 71.3$ & 3 DAY & 10. $\%$ & & $\begin{array}{l}\text { BET Dei } \\
\text { Thermal cross section most important. } \\
\text { Resonance integral also needed. } \\
\text { For interpretation of } \mathrm{N}^{\text {sos }}(\mathrm{n}, \mathrm{p}) \text { rluence } \\
\text { monitor data. }\end{array}$ \\
\hline${ }^{50} \mathrm{Co}$ & $\sigma_{n, y}(E)$ & 2 & META & STABL & 9.1 & 1 HR & 10.7 & & $\begin{array}{l}\text { BET Dei } \\
\text { Thermal cross section most important. } \\
\text { Resonance interal also needed. } \\
\text { For interpretation of } \mathrm{Ni}^{30}(\mathrm{n} . \mathrm{p}) \text { rluence } \\
\text { monitor data. }\end{array}$ \\
\hline
\end{tabular}


DOE-NDC Compilation of Requests for Nuclear Data 1982

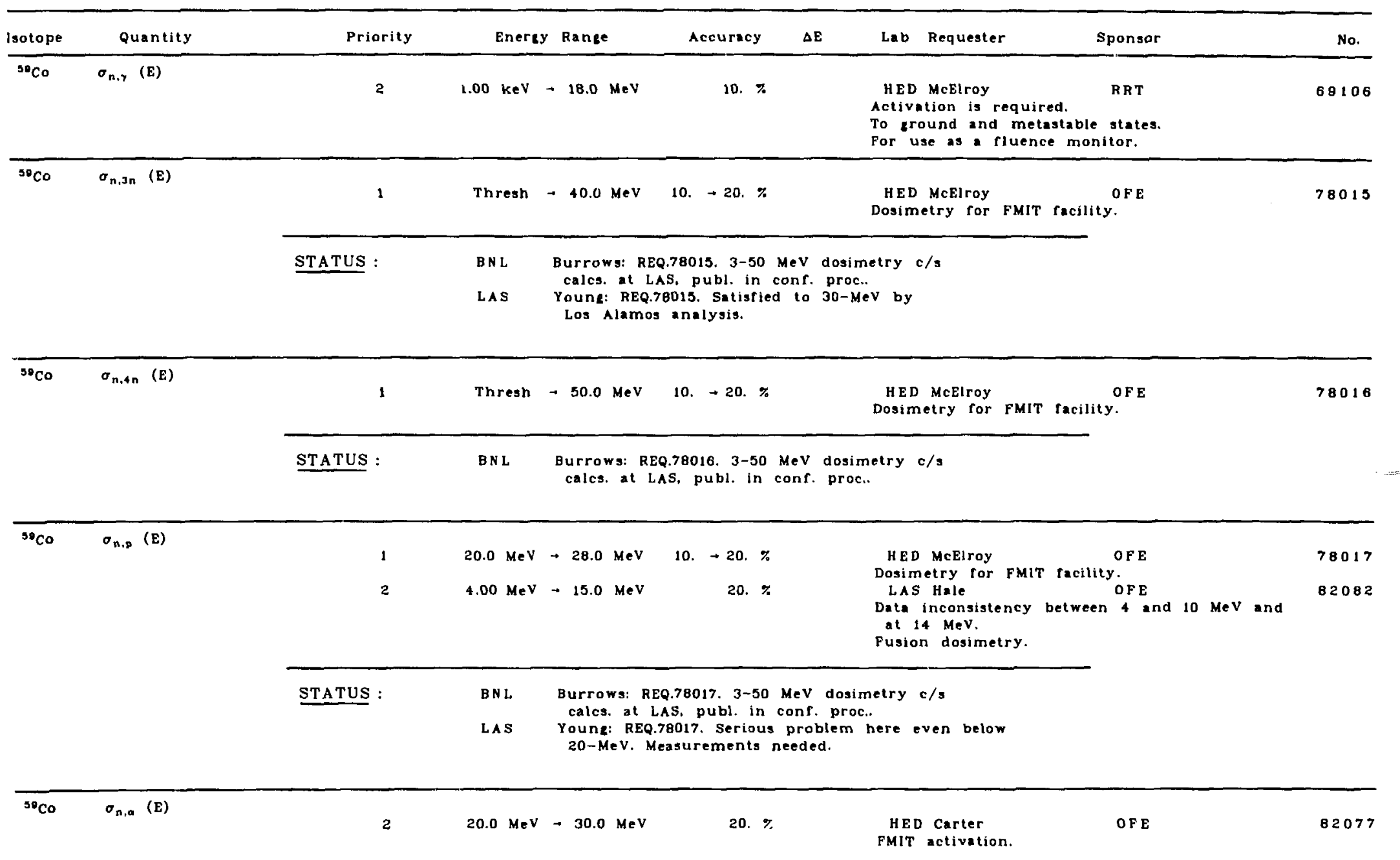


DOE-NDC Compilation of Requests for Nuclear Data 1982

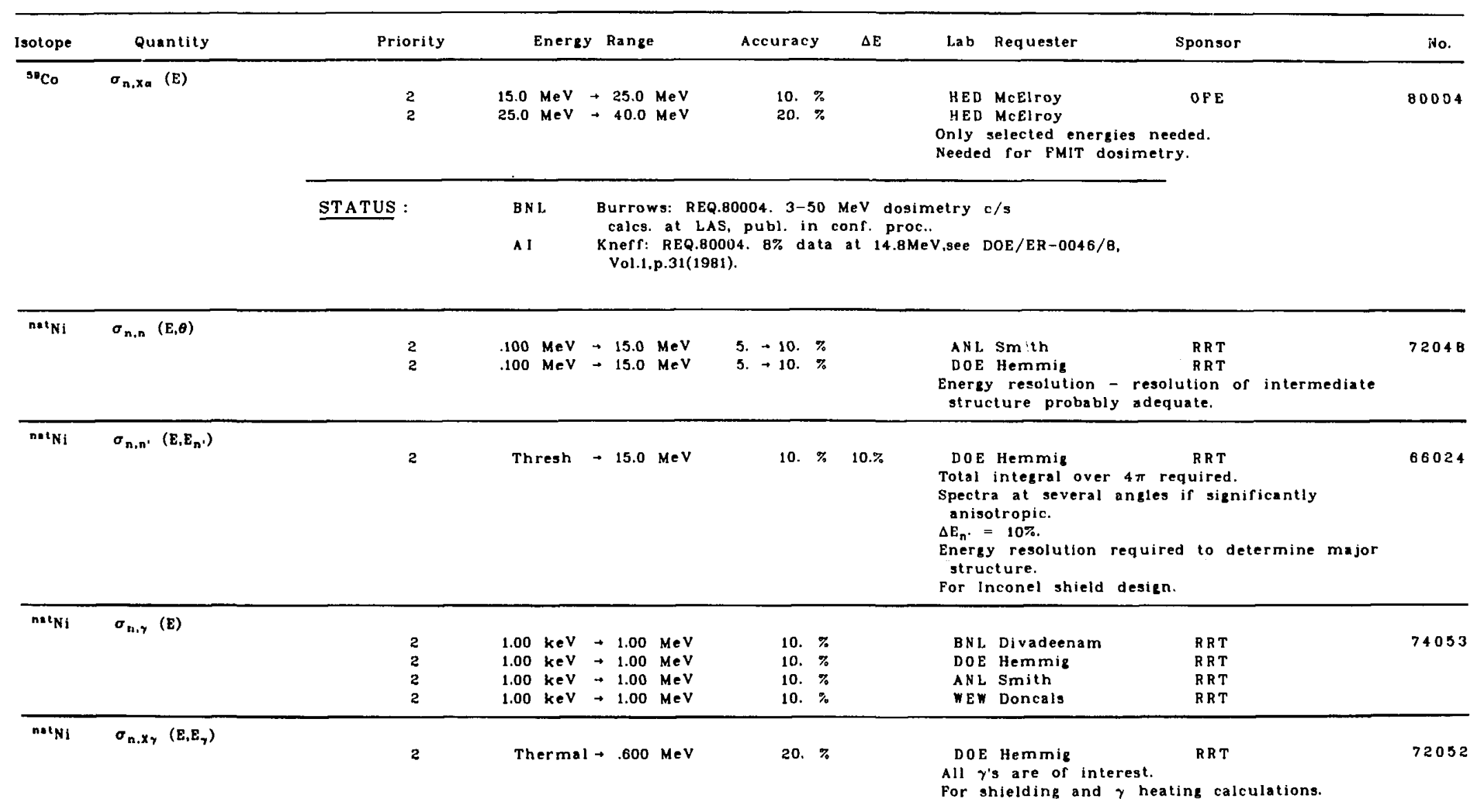


DOE-NDC Compilation of Requests for Nuclear Data 1982

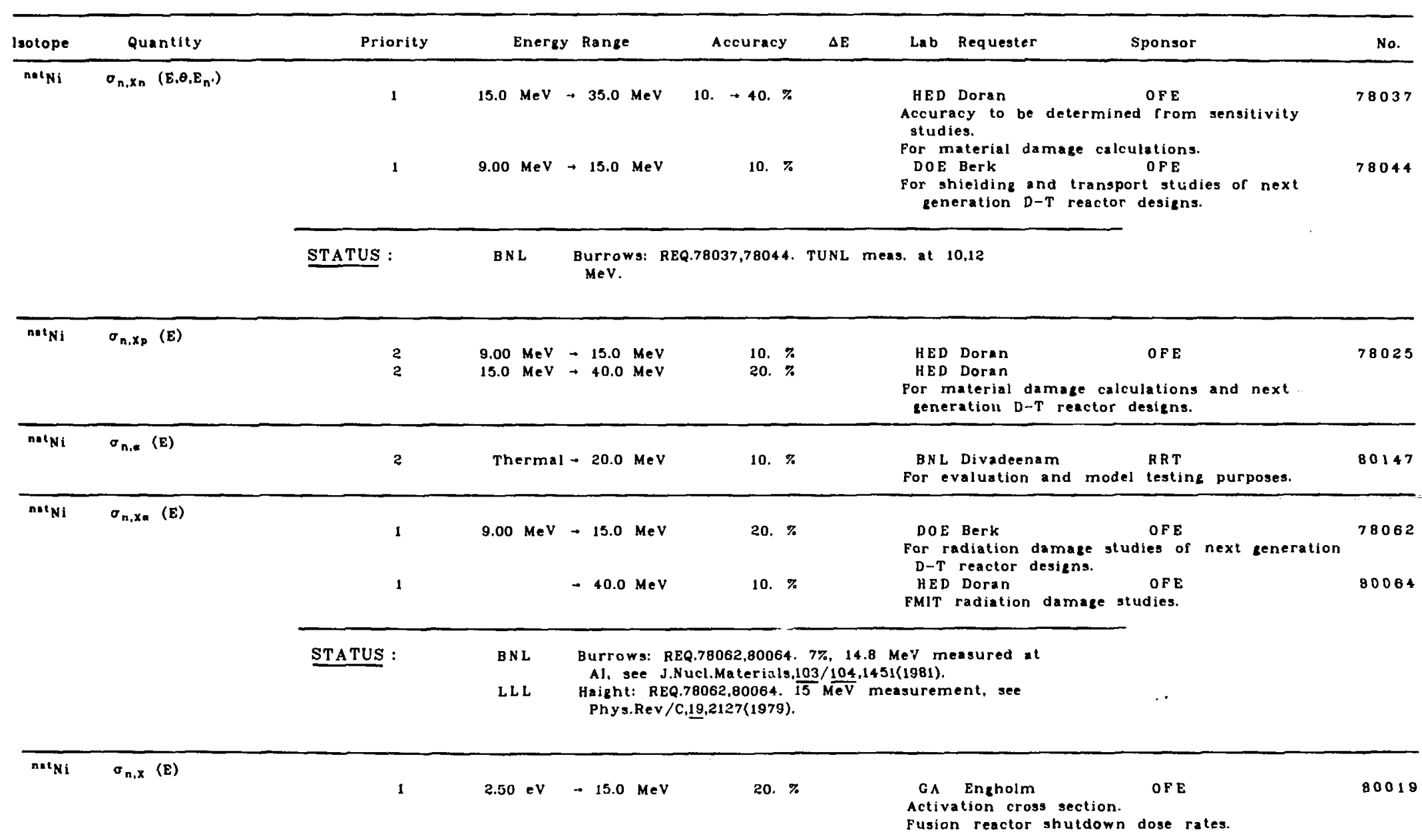


DOE-NDC Compilation of Requests for Nuclear Data 1982

\begin{tabular}{|c|c|c|c|c|c|c|c|c|c|c|}
\hline Isotope & Quantity & Priority & & Energy & y Range & & Accuracy & $\Delta E$ & Lab Requester & No. \\
\hline${ }^{58} \mathrm{Ni}$ & $\sigma_{n, \gamma}(E)$ & 2 & 1.00 & keV & $\rightarrow \quad 2.00$ & $\mathrm{MeV}$ & 10. $\%$ & & $\begin{array}{l}\text { BNL Divadeenam } \\
\text { For evaluation needs. } \\
\text { Average capture cross section. } \\
\text { For helium build-up via } \mathrm{Ni}^{30}(\mathrm{n}, \alpha) \text { reaction. }\end{array}$ & 80136 \\
\hline${ }^{38} \mathrm{Ni}$ & $\sigma_{n, 2 n}(E)$ & 1 & 20.0 & $\mathrm{MeV}$ & $\rightarrow 30.0$ & MeV & 10. $\rightarrow 20 . \%$ & & $\begin{array}{l}\text { HED Carter } \\
\text { FMIT activation. }\end{array}$ & 78020 \\
\hline${ }^{3 \theta} \mathrm{Ni}$ & $\sigma_{n, 3 n}(E)$ & 1 & 20.0 & $\mathrm{MeV}$ & $\rightarrow 40.0$ & $\mathrm{MeV}$ & 10. $\rightarrow 20 . \%$ & & $\begin{array}{l}\text { HED Carter } \\
\text { FMIT activation. }\end{array}$ & 78021 \\
\hline${ }^{88} \mathrm{Ni}$ & $\sigma_{n, p}(E)$ & $\begin{array}{l}3 \\
2\end{array}$ & $\begin{array}{r}\text { Thr } \\
2.00\end{array}$ & MeV & $\begin{array}{l}\rightarrow 15.0 \\
\rightarrow 10.0\end{array}$ & $\begin{array}{l}\mathrm{MeV} \\
\mathrm{MeV}\end{array}$ & 5. $\%$ & $5.0 \%$ & $\begin{array}{l}\text { BET Dei } \\
\text { DNR } \\
\text { For use as rluence monitor. } \\
\text { NBS McGarry } \\
\text { Required for reactor pressure vessel dosimetry. }\end{array}$ & $\begin{array}{l}72055 \\
82054\end{array}$ \\
\hline${ }^{38} \mathrm{Ni}$ & $\sigma_{n, 1}(E)$ & 2 & 15.0 & $\mathrm{MeV}$ & $\rightarrow 40.0$ & $\mathrm{MeV}$ & $20 . \%$ & & $\begin{array}{l}\text { HED McElroy } \\
\text { A } 11 \text { reactions leading to Co-56 are needed. } \\
\text { Needed for FMIT activation and dosimetry. }\end{array}$ & 80003 \\
\hline${ }^{50} \mathrm{Ni}$ & Resonance Parameters & $\begin{array}{l}2 \\
2\end{array}$ & .100 & $\mathrm{MeV}$ & $\begin{array}{l}+.100 \\
\rightarrow \quad .100 \\
\rightarrow \quad .700\end{array}$ & $\begin{array}{l}\mathrm{MeV} \\
\mathrm{MeV} \\
\mathrm{MeV}\end{array}$ & $\begin{array}{l}10 . \% \\
10 . \% \\
10 . \%\end{array}$ & & 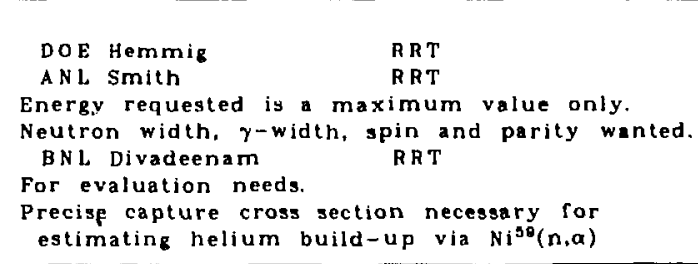 & 80135 \\
\hline${ }^{59} \mathrm{Ni}$ & $\sigma_{n, \alpha}(E)$ & 2 & $\begin{array}{c}\text { RADIC } \\
5.00\end{array}$ & $\begin{array}{l}\text { IOACTI } \\
0 \mathrm{keV}\end{array}$ & $\begin{array}{lr}\mathrm{VE} & 7.5 X \\
\rightarrow & 14.0\end{array}$ & $\begin{array}{l}5 \times(10 \cdots 4) \\
\mathrm{MeV}\end{array}$ & 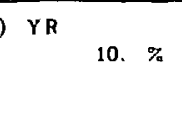 & & $\begin{array}{l}\text { BNL Divadeenam } \\
\text { a channel is open at zero neutron eneray. } \\
\text { Important for helium production. }\end{array}$ & 80128 \\
\hline
\end{tabular}


DOE-NDC Compilation of Requests for Nuclear Data 1982

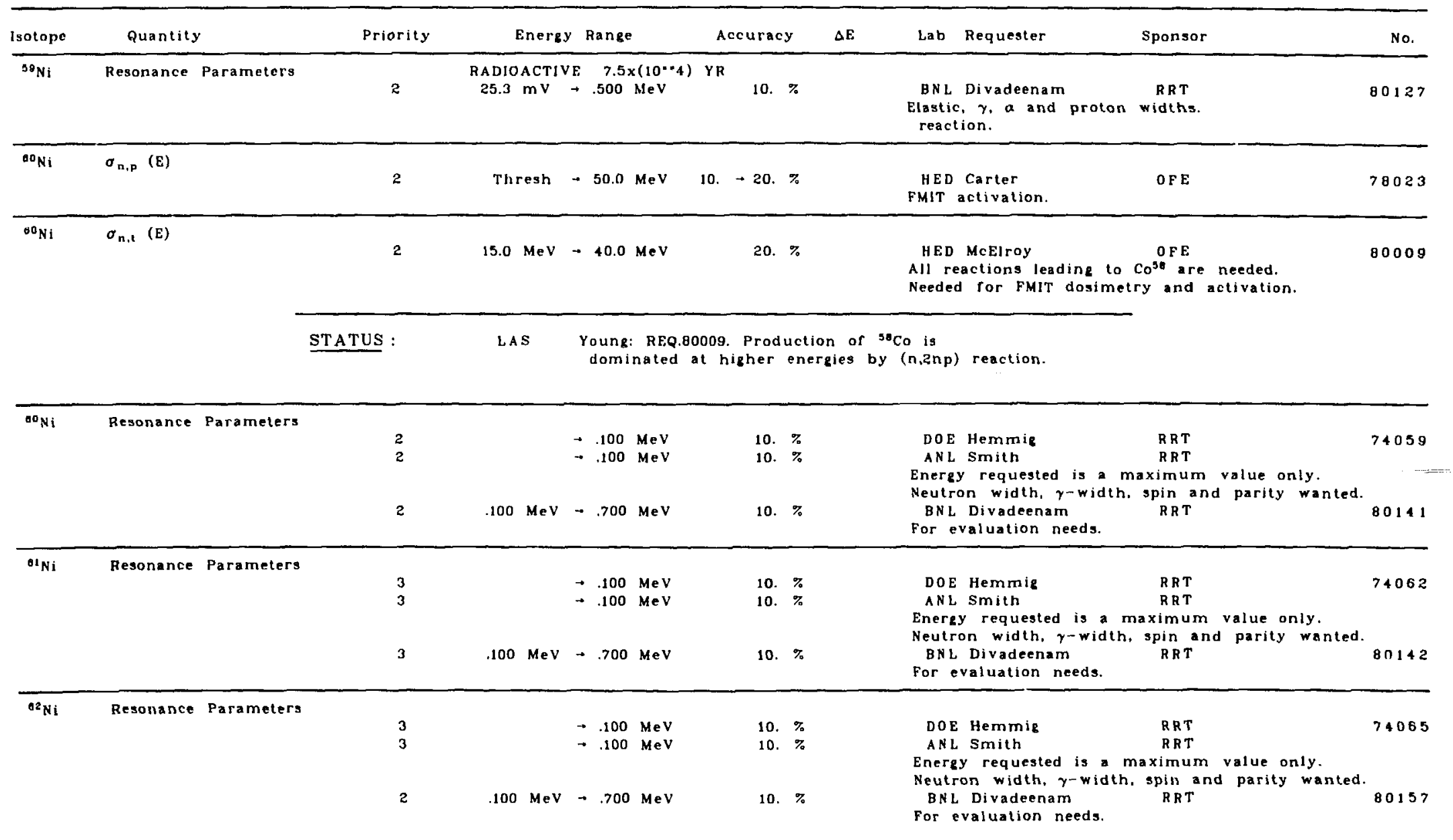




\begin{tabular}{|c|c|c|c|c|c|c|c|c|c|c|c|}
\hline Isotope & Quantity & Priority & Energy & $y \quad R$ & Range & & Accuracy & & $\Delta \mathrm{E}$ & Lab Requester & No. \\
\hline${ }^{83} \mathrm{Ni}$ & $\sigma_{n, p}(E)$ & 2 & $\begin{array}{l}\text { RADIOACTIV } \\
1.00 \mathrm{mV}\end{array}$ & & $\begin{array}{l}100 \\
10.0\end{array}$ & $\begin{array}{l}\text { YR } \\
\mathrm{MeV}\end{array}$ & 10. $\%$ & $\%$ & & $\begin{array}{l}\text { BET Dei } \\
\text { Flux monitor from } C u(n, p) \text { reaction. }\end{array}$ & 76053 \\
\hline${ }^{04} \mathrm{Ni}$ & Resonance Parameters & $\begin{array}{l}3 \\
3 \\
3\end{array}$ & $.100 \mathrm{MeV}$ & $\overrightarrow{\dot{ }}$ & $\begin{array}{l}.100 \\
.100 \\
.700\end{array}$ & $\begin{array}{l}\mathrm{MeV} \\
\mathrm{MeV} \\
\mathrm{MeV}\end{array}$ & $\begin{array}{l}\text { 10. } \% \\
\text { 10. } \% \\
\text { 10. }\end{array}$ & $\%$ & & $\begin{array}{l}\text { DOE Hemmig } \\
\text { ANL Smith } \\
\text { RRT } \\
\text { Energy requested is a maximum value only. } \\
\text { Neutron width, q-width, spin and parity wanted. } \\
\text { BNL Divadeenam } \\
\text { For evaluation needs. }\end{array}$ & $\begin{array}{l}74068 \\
80143\end{array}$ \\
\hline natcu & $\sigma_{\gamma, n}(E)$ & 2 & Thresh & $\rightarrow$ & 20.0 & $\mathrm{MeV}$ & 30. & $\%$ & $5 . \%$ & $\begin{array}{l}\text { MDD Driemeyer OFE } \\
\text { Determination of photoneutron activation in EBT- } \\
\text { Needed in assessing potential activation problems } \\
\text { in the uperade phase of EBT-P operation. }\end{array}$ & $\begin{array}{l}82044 \\
P .\end{array}$ \\
\hline$n=t c u$ & $\sigma_{n, t o t}(E)$ & 2 & $13.0 \mathrm{MeV}$ & $\rightarrow$ & 15.0 & $\mathrm{MeV}$ & 1.0 & 7 & & $\begin{array}{l}\text { LAS Young } \\
\text { For radiation damage and heating calculations in } \\
\text { the toroidal field coils of a rusion device. } \\
\text { LAS Young } \\
\text { For radiation damage and heating calculations in } \\
\text { the toroidal field coils of a fusion device. }\end{array}$ & $\begin{array}{l}82037 \\
82038\end{array}$ \\
\hline
\end{tabular}

the toroidal field coils of fusion device.

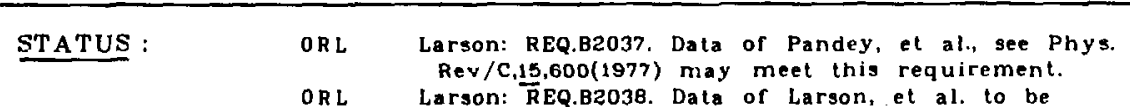

ORL Larson: REQ.B2038. Data of Larson, et al. to be

published should meet this request.

natcu $\sigma_{n, n}(E)$

the toroidal field coils of a cusion device.

STATUS : ORL Larson: REQ.82039. Presently avallable data is being
evaluated.


DOE-NDC Compilation of Requests for Nuclear Data 1982

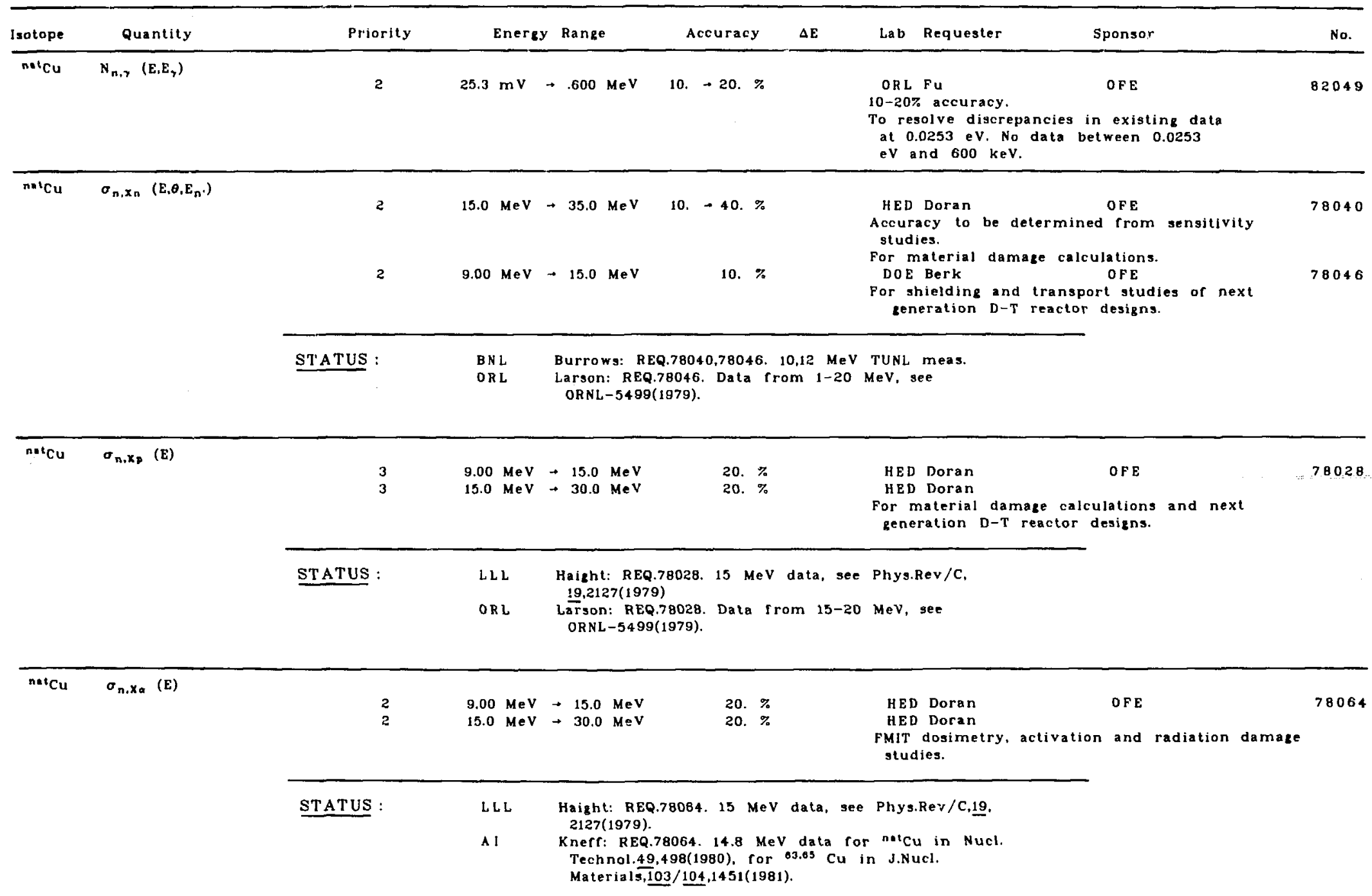


DOE-NDC Compilation of Requests for Nuclear Data 1982

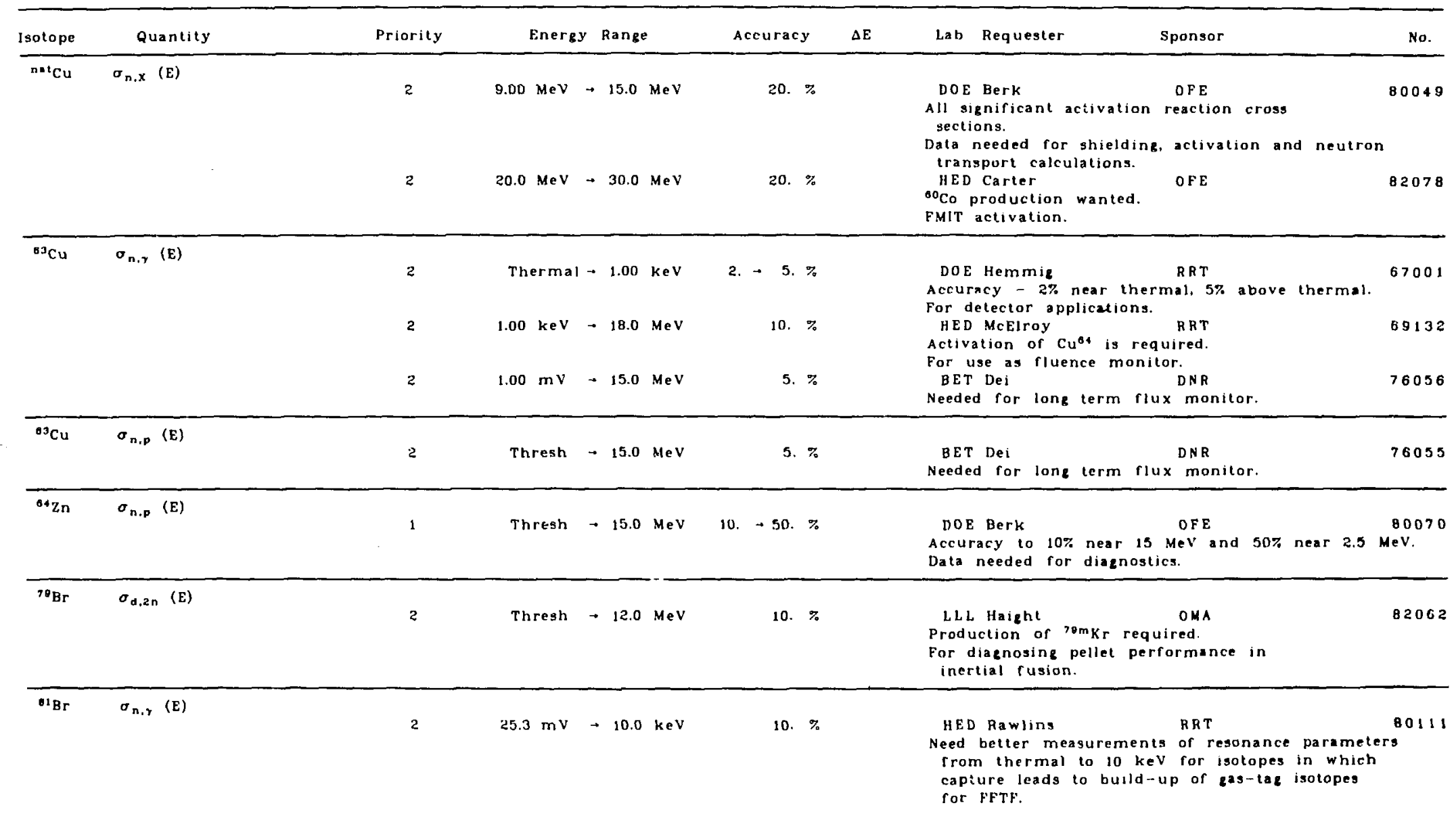


DOE-NDC Compilation of Requests for Nuclear Data 1982

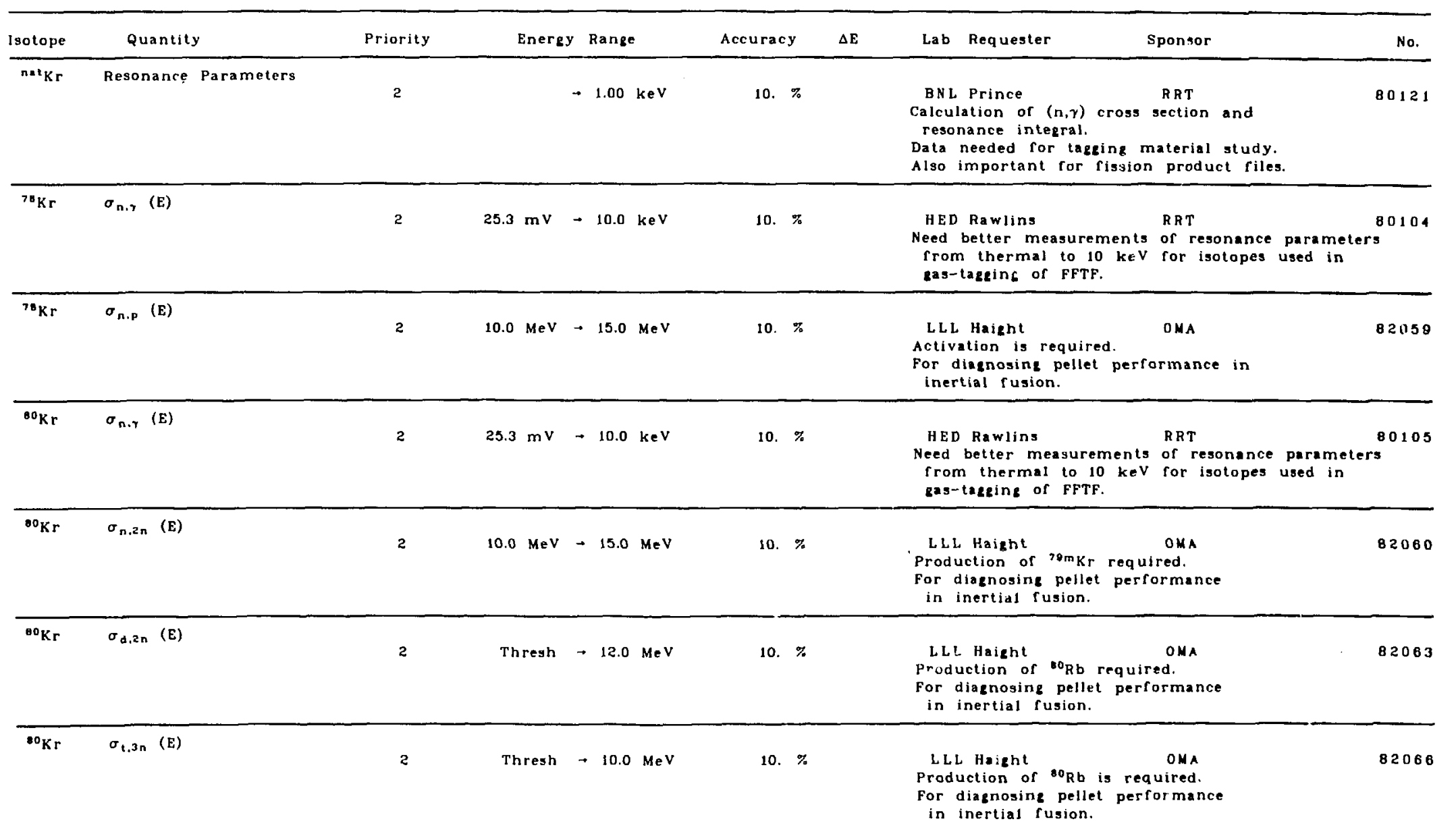


DOE-NDC Compilation of Requests for Nuclear Data 1982

\begin{tabular}{|c|c|c|c|c|c|c|c|c|c|}
\hline Isotope & Quentity & Priority & Enerzy & y Range & & Accuracy & $\Delta \mathrm{E}$ & Lab Requester & No. \\
\hline${ }^{12} \mathrm{Kr}$ & $\sigma_{n, \gamma}(E)$ & 2 & $\begin{array}{l}40.0 \mathrm{eV} \\
25.3 \mathrm{mV}\end{array}$ & +10.0 & keV & $\begin{array}{l}\text { 10. } \% \\
10 . \%\end{array}$ & & $\begin{array}{l}\text { NBS Bowman } \\
\text { Values for a rew higher resonances also needed. } \\
\text { Necded to ground. First and second excited states } \\
\text { for } \text {-ray laser. } \\
\text { HED Rawlins } \\
\text { Need better measurements of resonance parameters } \\
\text { rrom thermal to } 10 \mathrm{keV} \text { for isotopes used in } \\
\text { gas-tagging of FFTF. }\end{array}$ & $\begin{array}{l}76116 \\
80106 \\
5\end{array}$ \\
\hline${ }^{12} \mathrm{Kr}$ & $\sigma_{d .2 n}(\varepsilon)$ & 2 & Thresh & $\rightarrow \quad 12.0$ & Mev & 20. $\%$ & & $\begin{array}{l}\text { LLL Haight } \\
\text { Production of }{ }^{2} R \text { b is required. } \\
\text { For diagnosing pellet performance in } \\
\text { inertial fusion. }\end{array}$ & 82064 \\
\hline${ }^{2} \mathrm{~K} \mathrm{Kr}$ & $\sigma_{1,3 n}(E)$ & $z$ & Thresh & $\rightarrow 10.0$ & $\mathrm{MeV}$ & 10. $\%$ & & $\begin{array}{l}\text { LLL Haight } \\
\text { Production of }{ }^{02} \mathrm{Rb} \text { is required. } \\
\text { For diagnosing pellet performance } \\
\text { in inertial fusion. }\end{array}$ & 82067 \\
\hline${ }^{13} \mathrm{Kr}$ & Resonance Paramelers & $\begin{array}{l}2 \\
2\end{array}$ & $\begin{array}{l}1.00 \mathrm{mv} \\
1.00 \mathrm{mv}\end{array}$ & $\begin{array}{l}\rightarrow \quad 1.00 \\
\rightarrow \quad 1.00\end{array}$ & $\begin{array}{l}\text { keV } \\
\text { kev }\end{array}$ & $\begin{array}{l}10 . \% \\
10 . \%\end{array}$ & & $\begin{array}{lc}\text { BET Dei } & \text { DNR } \\
\text { KAP Feiner } & \text { DNR } \\
\text { For fission product absorption calculation. }\end{array}$ & 67190 \\
\hline$\omega_{K r}$ & $\sigma_{d, 2 n}(E)$ & 2 & Thresh & $\rightarrow \quad 12.0$ & MeV & 10. $\%$ & & $\begin{array}{l}\text { LLL Haight } \\
\text { Production of }{ }^{\circ} R \text { is is required. } \\
\text { For diagnosing pellet performance } \\
\text { in inertial fusion. }\end{array}$ & B2065 \\
\hline${ }^{10} \mathrm{Kr}$ & $\sigma_{t, 3 n}(E)$ & 2 & Thresh & - 10.0 & $\mathrm{MeV}$ & $10 . \%$ & & $\begin{array}{l}\text { LLL Haight } \\
\text { Production or som } \mathrm{Rb} \text { is required. } \\
\text { For diagnosing pellet performance } \\
\text { in inertial rusion. }\end{array}$ & 82068 \\
\hline
\end{tabular}




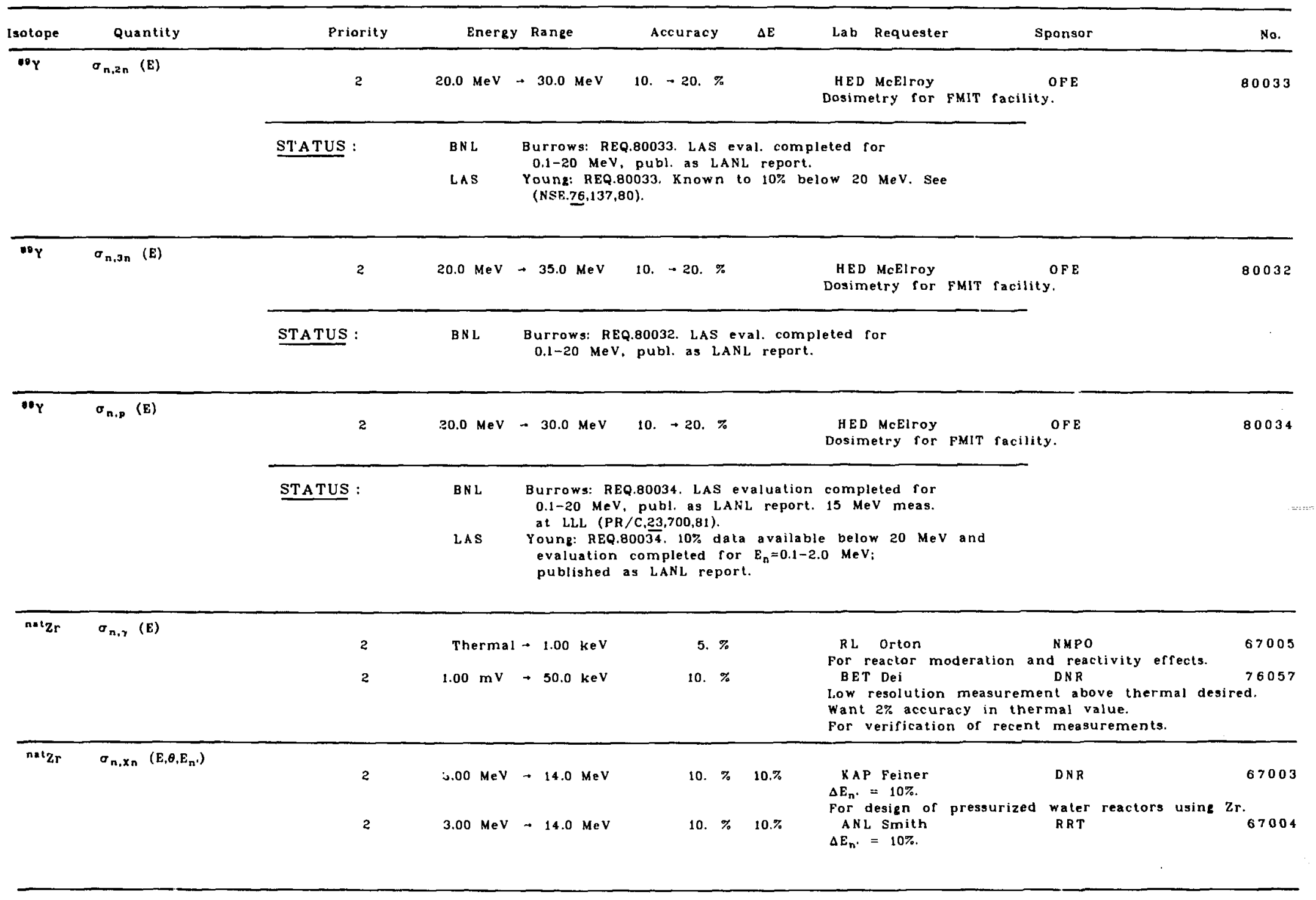


DOE-NDC Compilation of Requests for Nuclear Data 1982

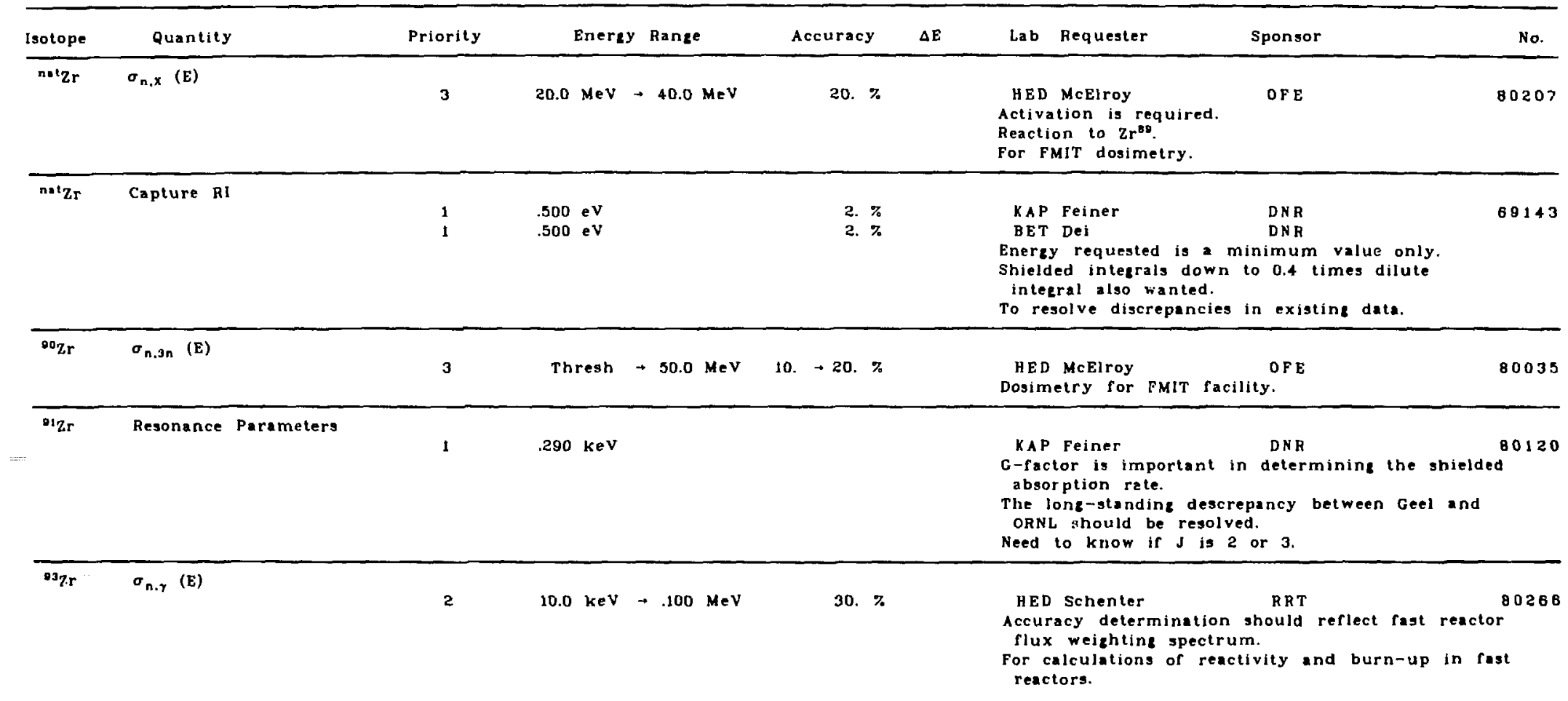


DOE-NDC Compilation of Requests for Nuclear Data 1982

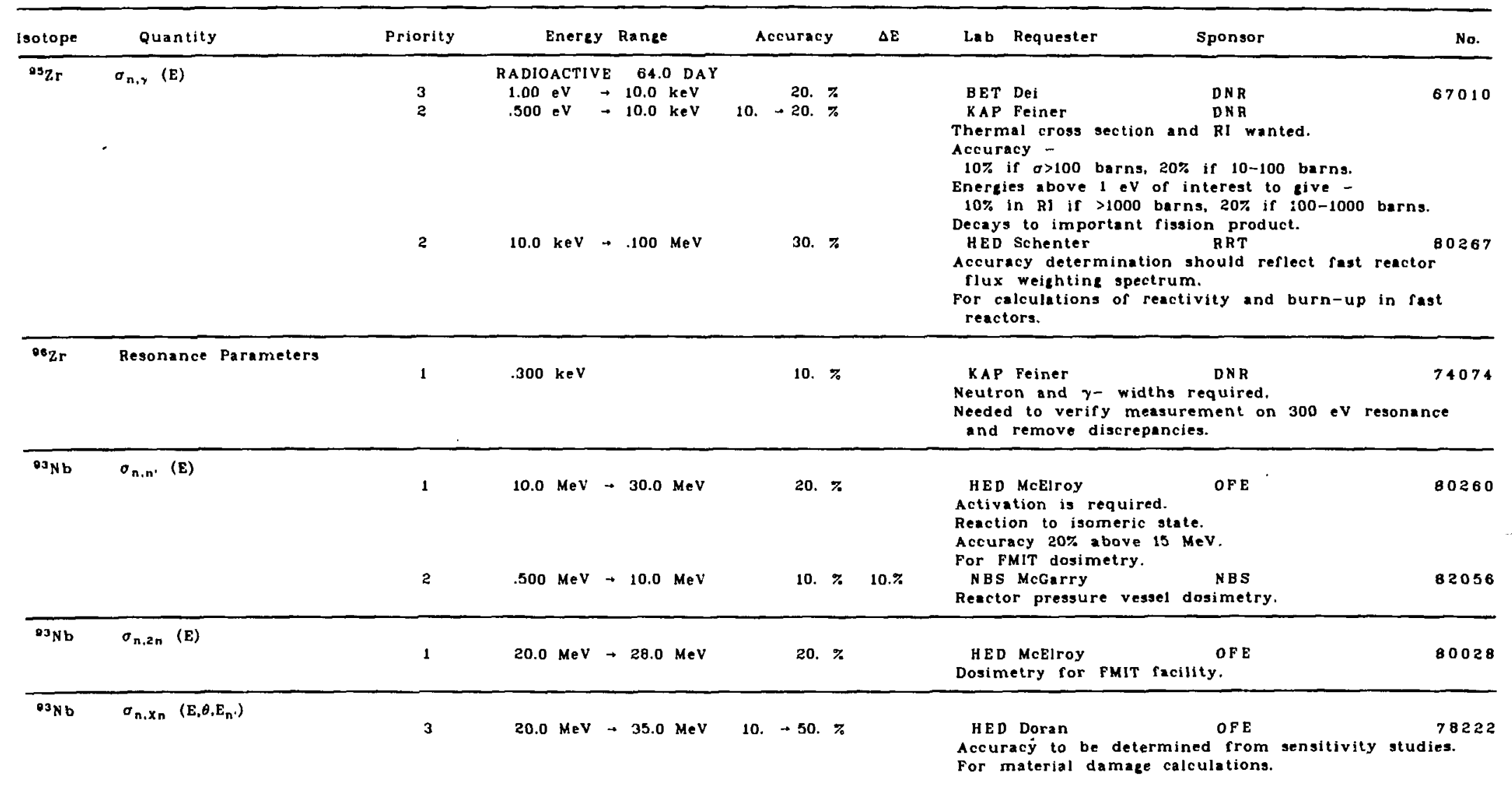


DOE-NDC Compilation of Requests for Nuclear Data 1982

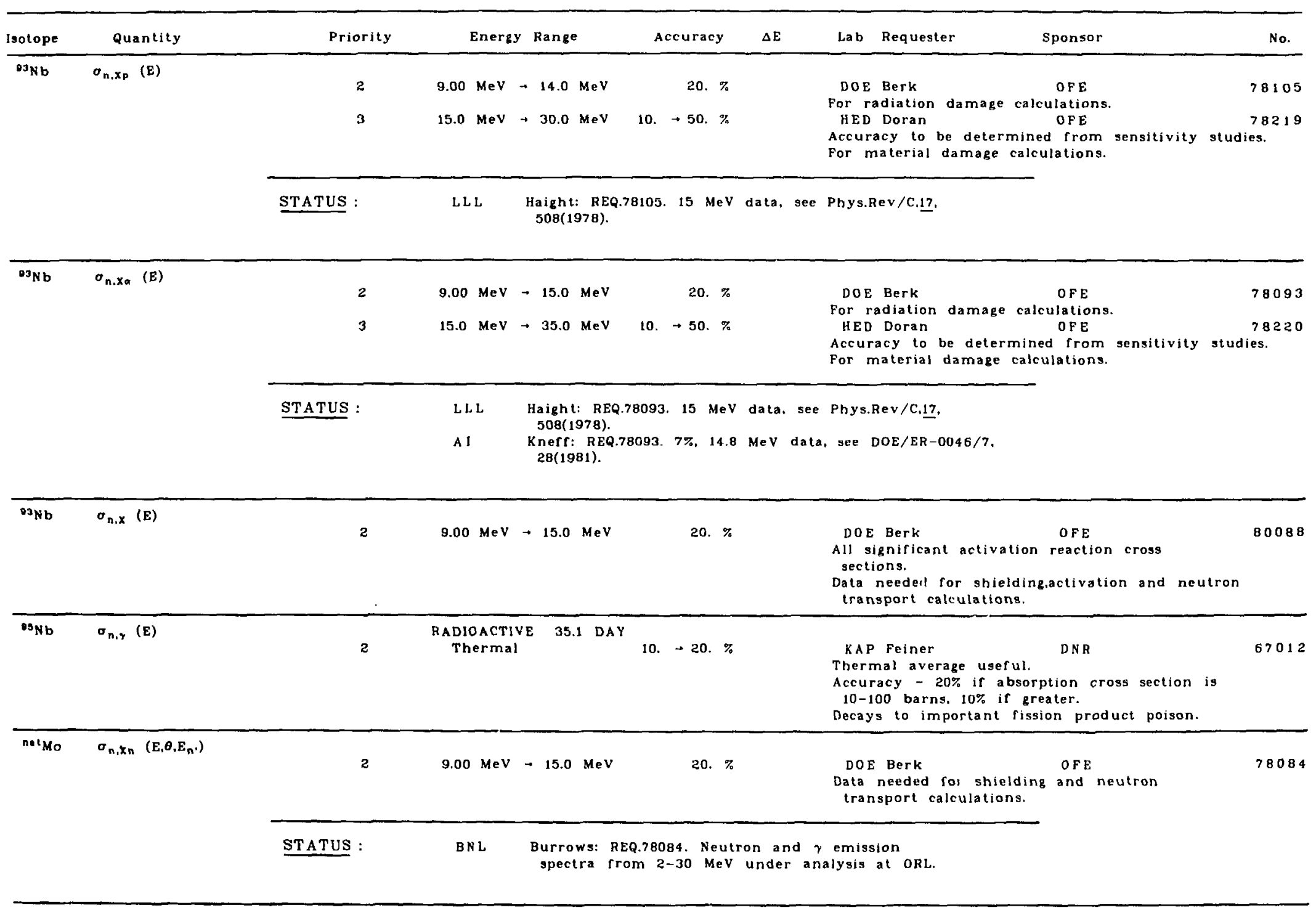


DOE-NDC Compilation of Requests for Nuclear Data 1982

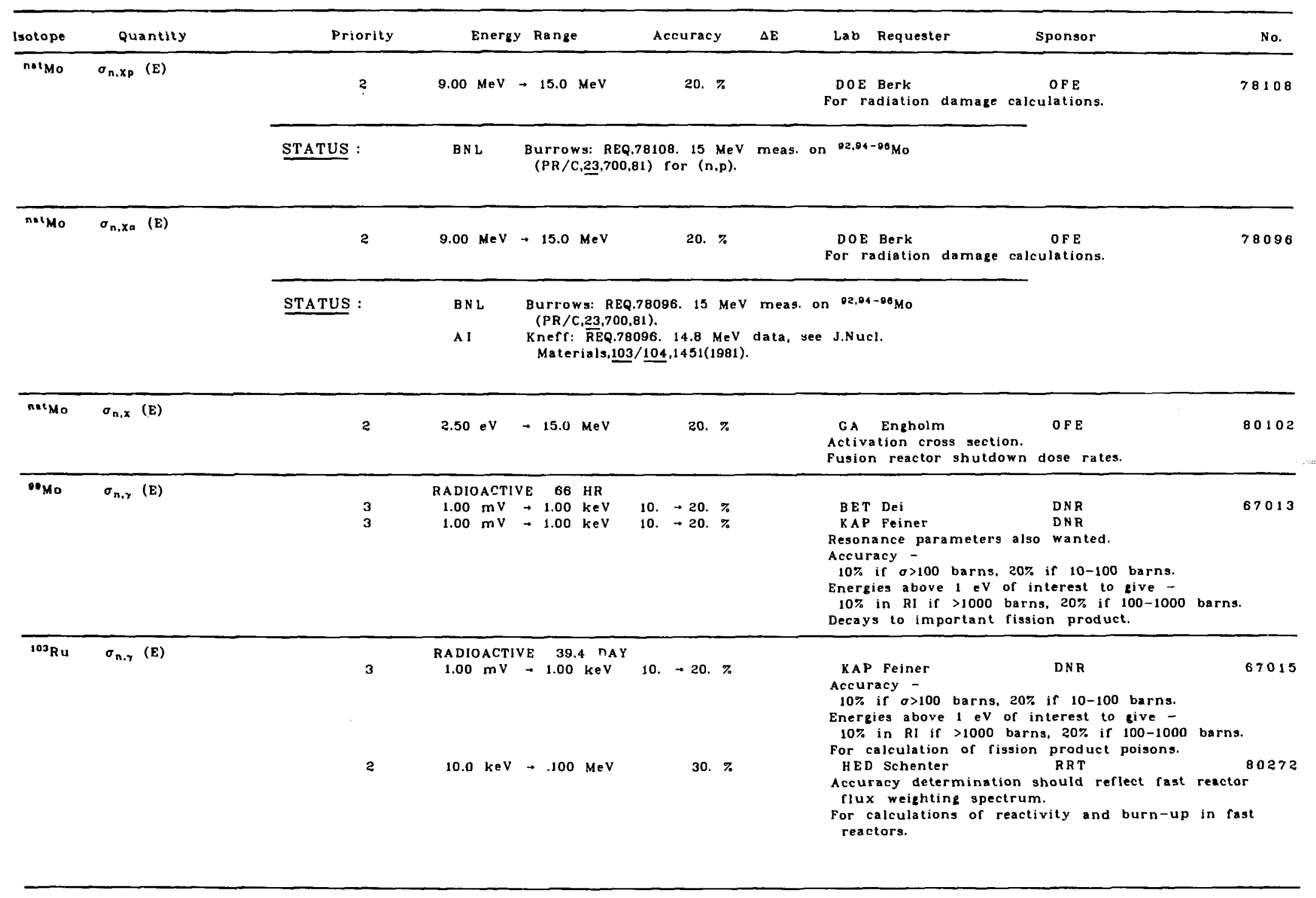


DOE-NDC Compilation of Requests for Nuclear Data 1982

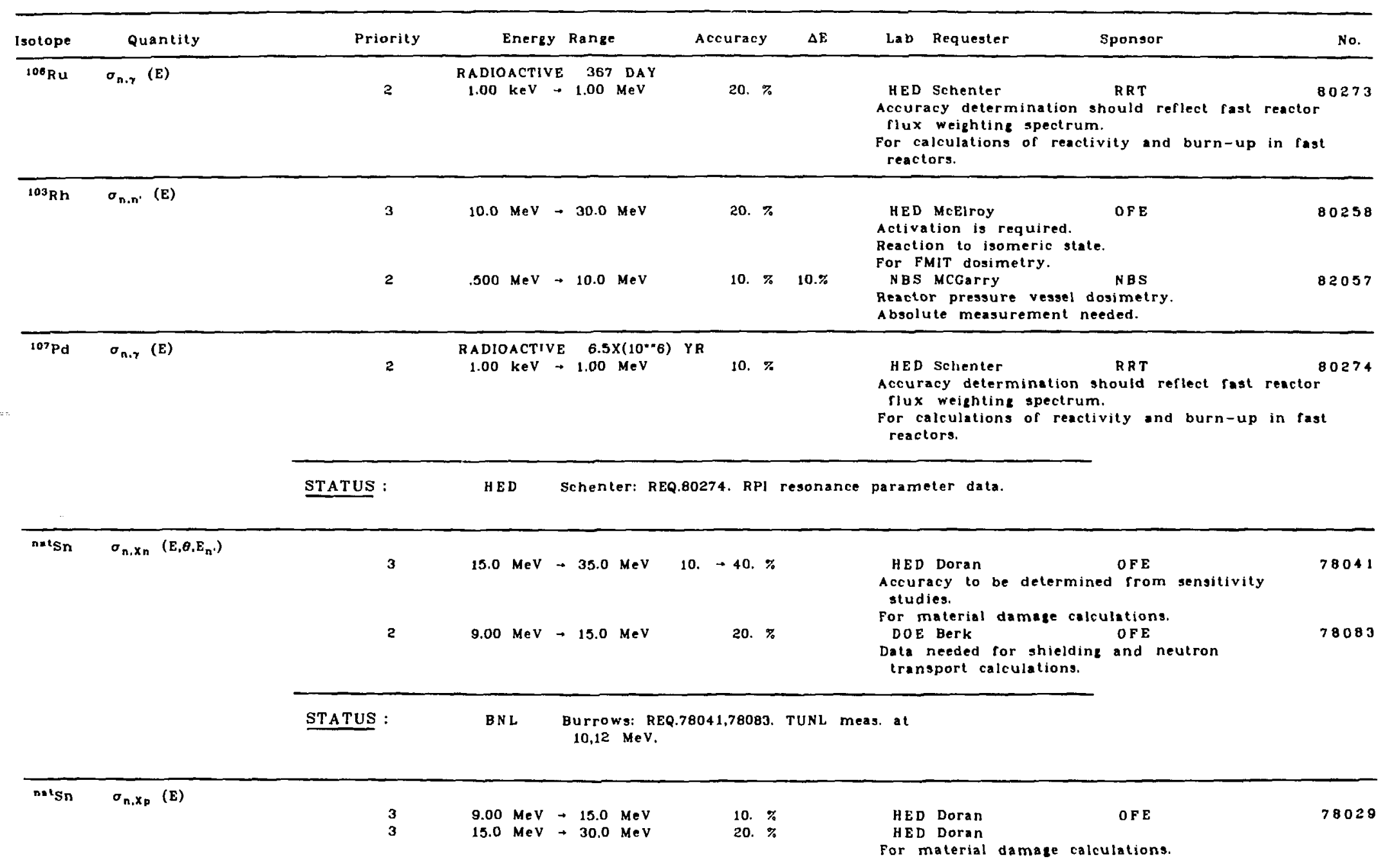


DOE-NDC Compilation of Requests for Nuclear Data 1982

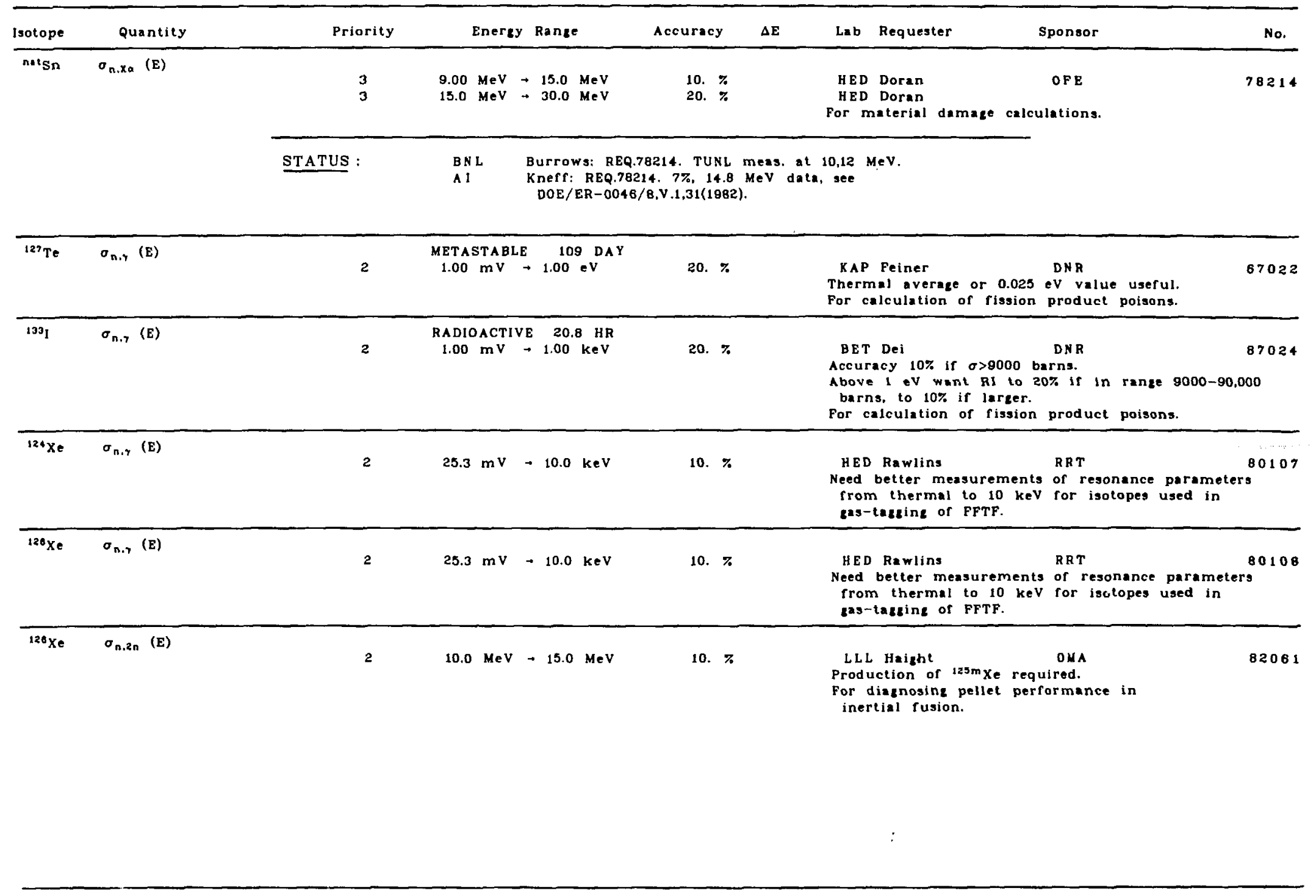


DOE-NDC Compilation of Requests for Nuclear Data 1982

\begin{tabular}{|c|c|c|c|c|c|c|c|}
\hline Isotope & Quantity & Priority & Energy & y Range & Accuracy & $\Delta E$ & Lab Requester \\
\hline${ }^{129} \times e^{2}$ & $\sigma_{n, \gamma}(E)$ & 2 & $25.3 \mathrm{mV}$ & $\rightarrow 10.0 \mathrm{keV}$ & 10. $\%$ & & $\begin{array}{l}\text { HED Rawlins } \\
\text { Need better measurements of resonance parameters } \\
\text { from thermal to } 10 \mathrm{keV} \text { for isotopes used in } \\
\text { gas-tagging of FFTF. }\end{array}$ \\
\hline${ }^{120} \mathrm{Xe}$ & $\sigma_{n, \gamma}(E)$ & 2 & $25.3 \mathrm{mV}$ & $\rightarrow \quad 10.0 \mathrm{keV}$ & 10. $\%$ & & $\begin{array}{l}\text { HED Rawlins } \\
\text { Need better measurements of resonance parameters } \\
\text { from thermal to } 10 \mathrm{keV} \text { for isotopes used in } \\
\text { gas-tagging of FFTF. }\end{array}$ \\
\hline${ }^{131} \times \mathrm{Xe}$ & $\sigma_{n, \gamma}(E)$ & 2 & $1.00 \mathrm{keV}$ & $\rightarrow 1.00 \mathrm{MeV}$ & 20. $\%$ & & 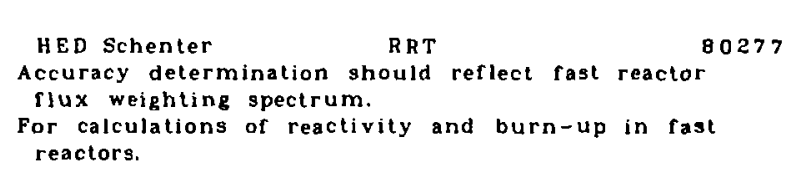 \\
\hline${ }^{133} \times \mathrm{Xe}$ & $\sigma_{n, \gamma}(E)$ & 2 & $\begin{array}{l}\text { RADIOACTIV } \\
1.00 \mathrm{mV}\end{array}$ & $\begin{array}{l}\text { VE } \quad 5.29 \text { DAY } \\
\rightarrow \quad 5.00 \mathrm{keV}\end{array}$ & 10. $\%$ & & $\begin{array}{l}\text { BET Dei } \\
\text { Thermal cross section and RI wanted. } \\
\text { For fission product poison calculations. }\end{array}$ \\
\hline${ }^{135} \mathrm{Xe}$ & $\sigma_{n, \gamma}(E)$ & $\begin{array}{l}2 \\
2 \\
2\end{array}$ & $\begin{array}{l}\text { RADIOACTIV } \\
1.00 \mathrm{mV} \\
1.00 \mathrm{mV} \\
5.00 \mathrm{eV}\end{array}$ & $\begin{array}{l}\quad 9.17 \mathrm{HR} \\
\overrightarrow{ } \quad 5.00 \mathrm{eV} \\
\rightarrow \quad 5.00 \mathrm{eV} \\
\rightarrow \quad 5.00 \mathrm{keV}\end{array}$ & $\begin{array}{l}\text { 2. } \% \\
\text { 3. } \% \\
\text { 5. } \%\end{array}$ & & $\begin{array}{lc}\text { BET Dei } & \text { DNR } \\
\text { KAP Feiner } & \text { DNR } \\
\text { BET Dei } & \text { ONR } \\
\text { Thermal cross section wanted to 2\%. } \\
\text { For rission product poison calculations. }\end{array}$ \\
\hline${ }^{135} \mathrm{xe}$ & $\sigma_{n, X y}\left(E, E_{\gamma}\right)$ & 2 & $\begin{array}{l}\text { RADIOACTIV } \\
\text { Thermal }\end{array}$ & VE $9.17 \mathrm{HR}$ & $10 . \rightarrow 20 . \%$ & & $\begin{array}{l}\text { KAP Feiner } \\
\text { For } \gamma \text { energies } 1-8 \mathrm{MeV} \text {. DNR } \\
\gamma \text {-energy resolution }-10-20 \% \\
\text { For } \gamma \text { shielding and heat calculations. }\end{array}$ \\
\hline${ }^{135} \mathrm{Cs}$ & $\sigma_{n_{;} \gamma}(E)$ & 2 & $\begin{array}{l}\text { RADIOACTIV } \\
1.00 \mathrm{keV}\end{array}$ & $\begin{array}{l}\text { VE } 3.0 \times\left(10^{.06}\right) \\
\rightarrow \quad 1.00 \mathrm{MeV}\end{array}$ & $20 . \%$ & & 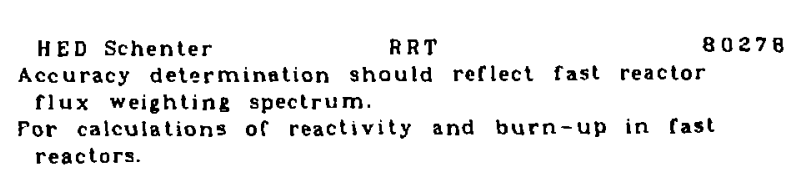 \\
\hline
\end{tabular}




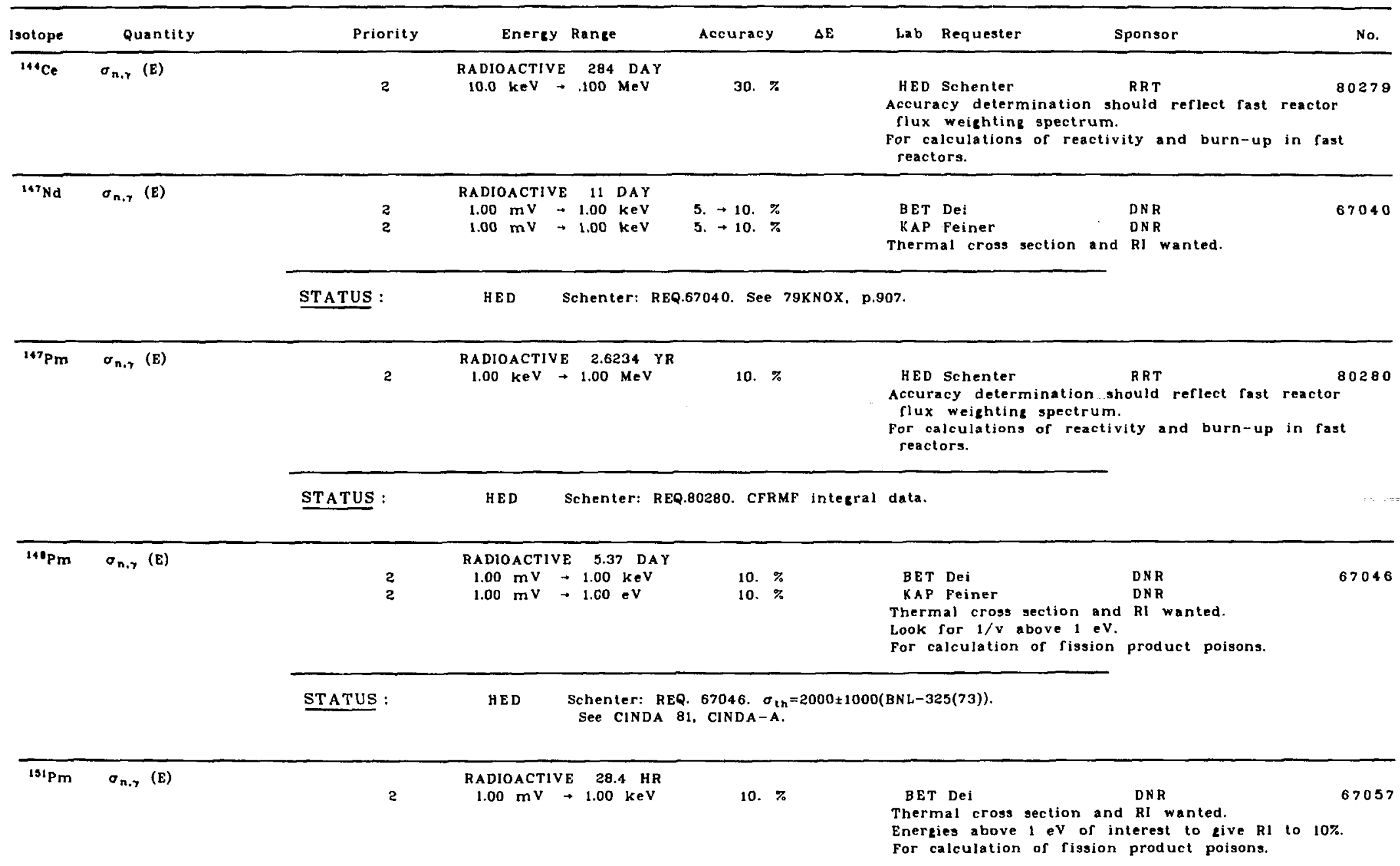


DOE-NDC Compilation of Requests for Nuclear Data 1982

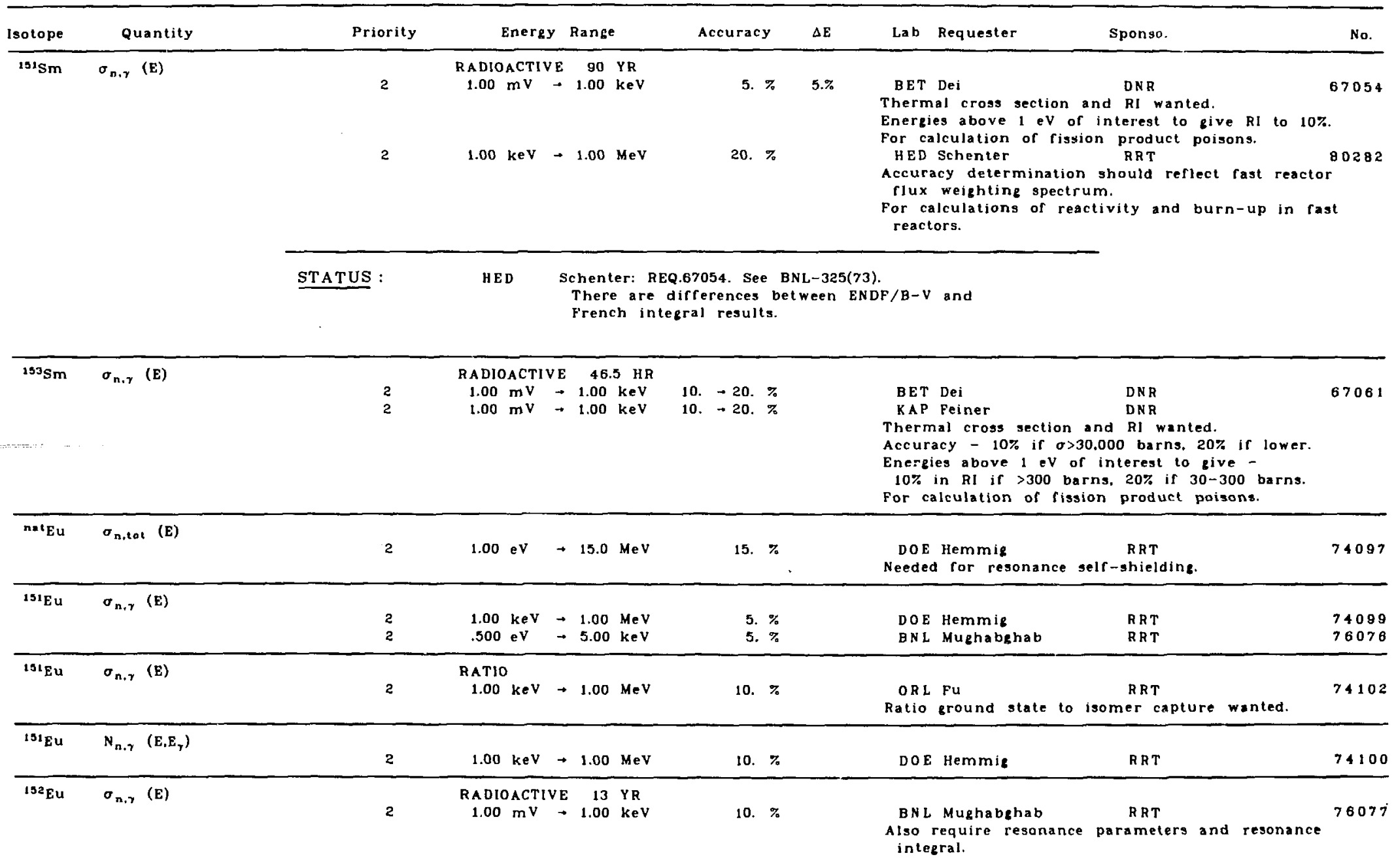


DOE-NDC Compilation of Requests for Nuclear Data 1982

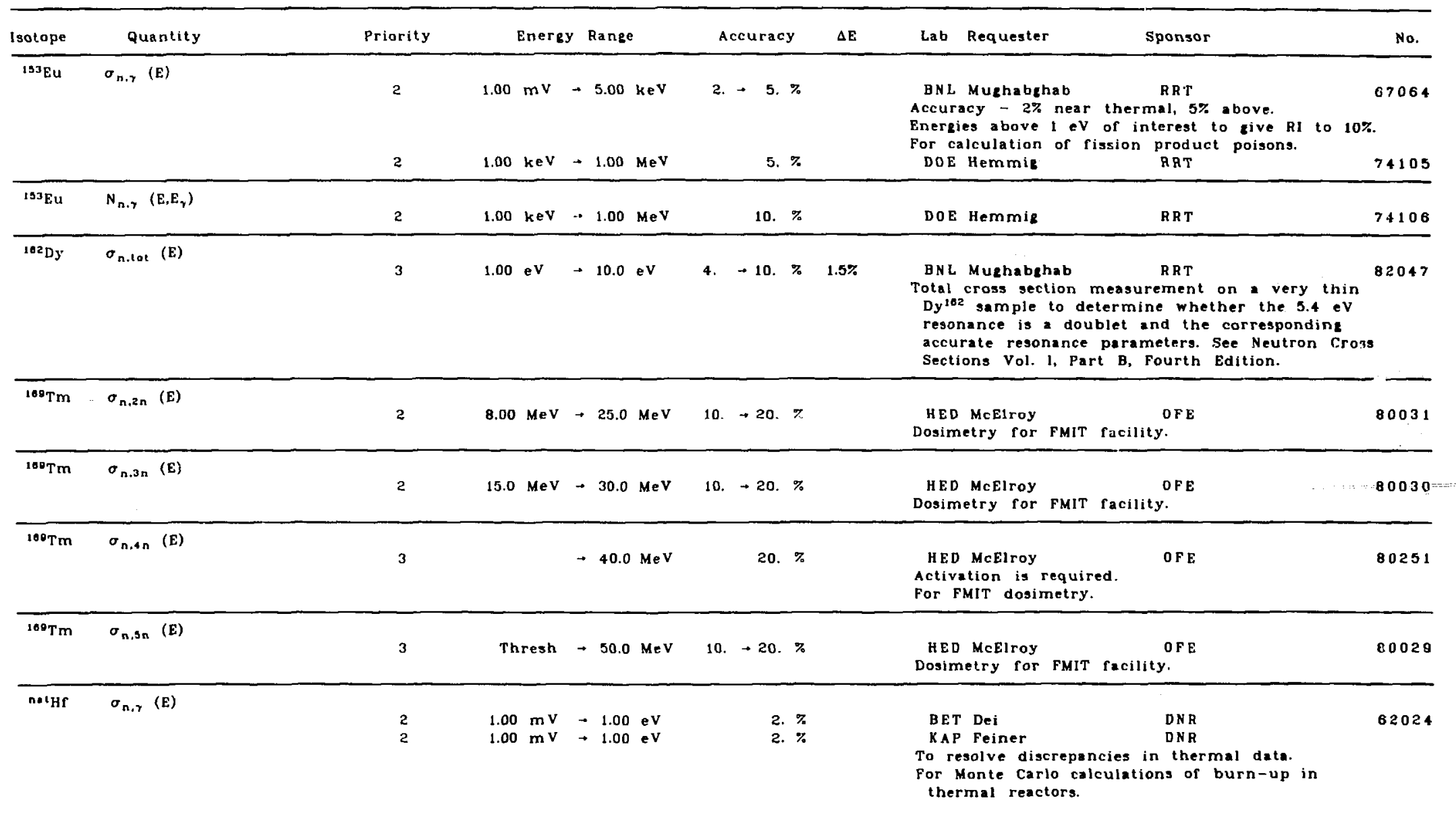


DOE-NDC Compilation of Requests for Nuclear Data 1982

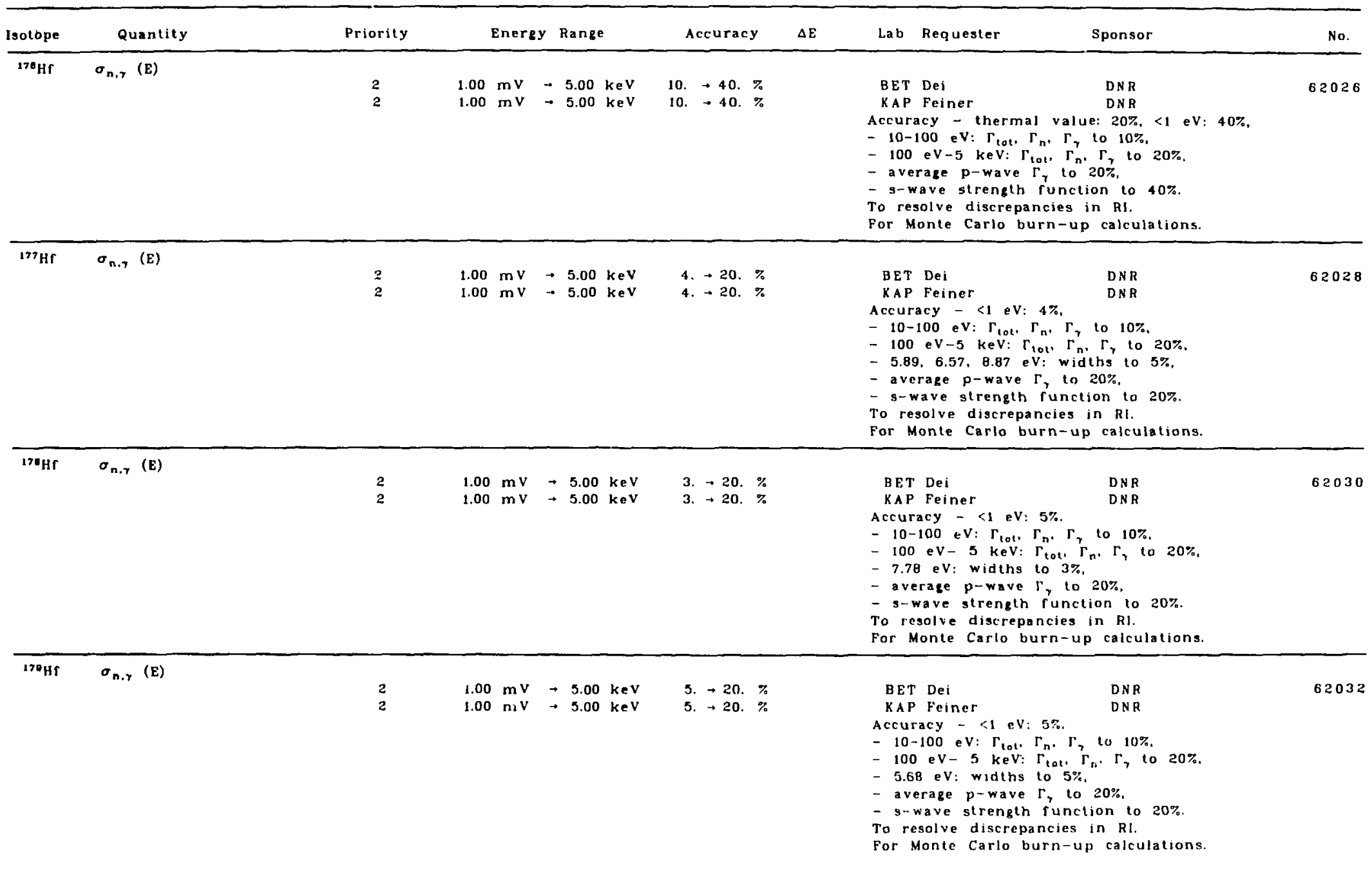


DOE-NDC Compilation of Requests for Nuclear Data 1982

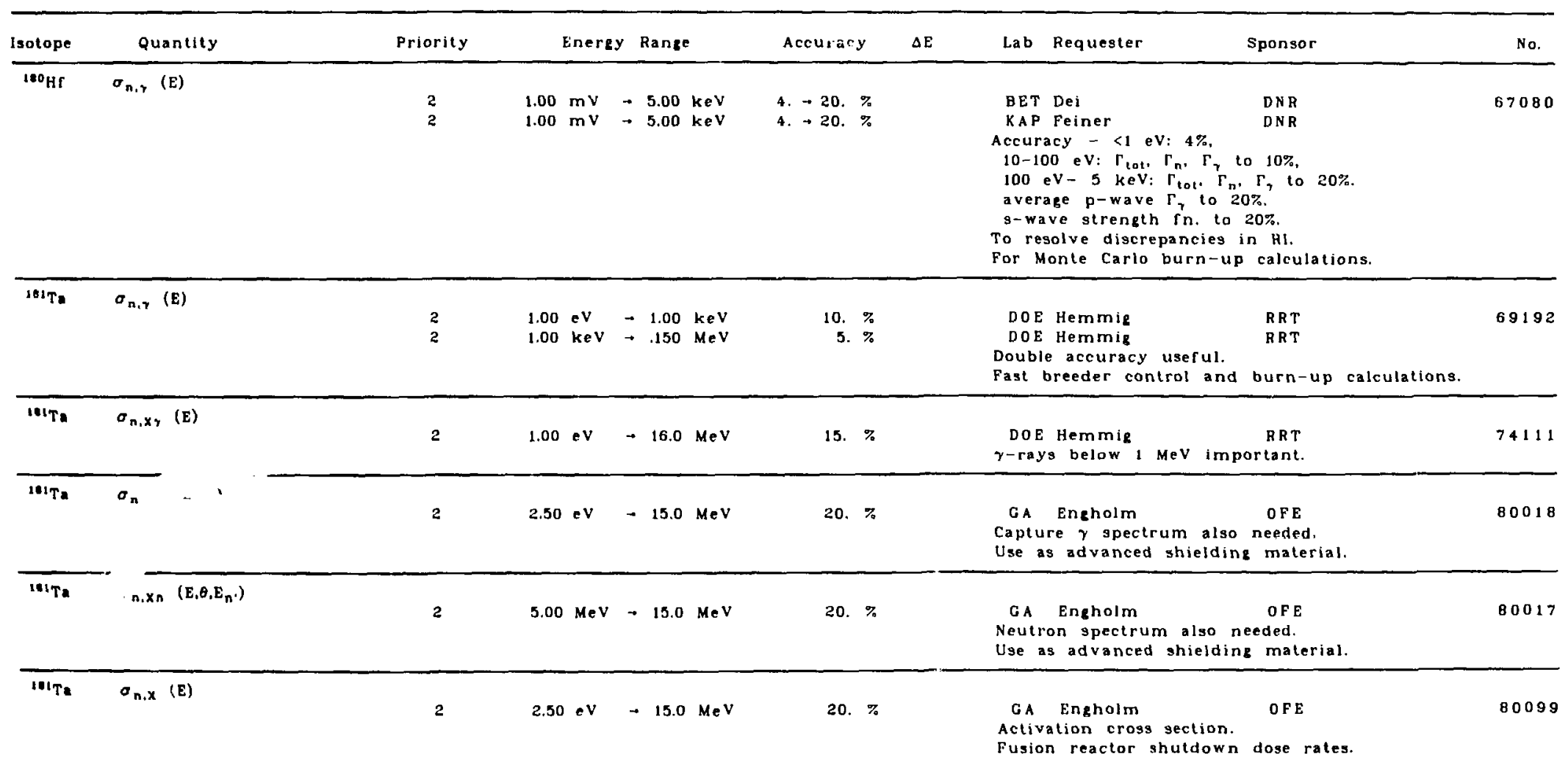


DOE-NDC Compilation of Requests for Nuclear Data 1982

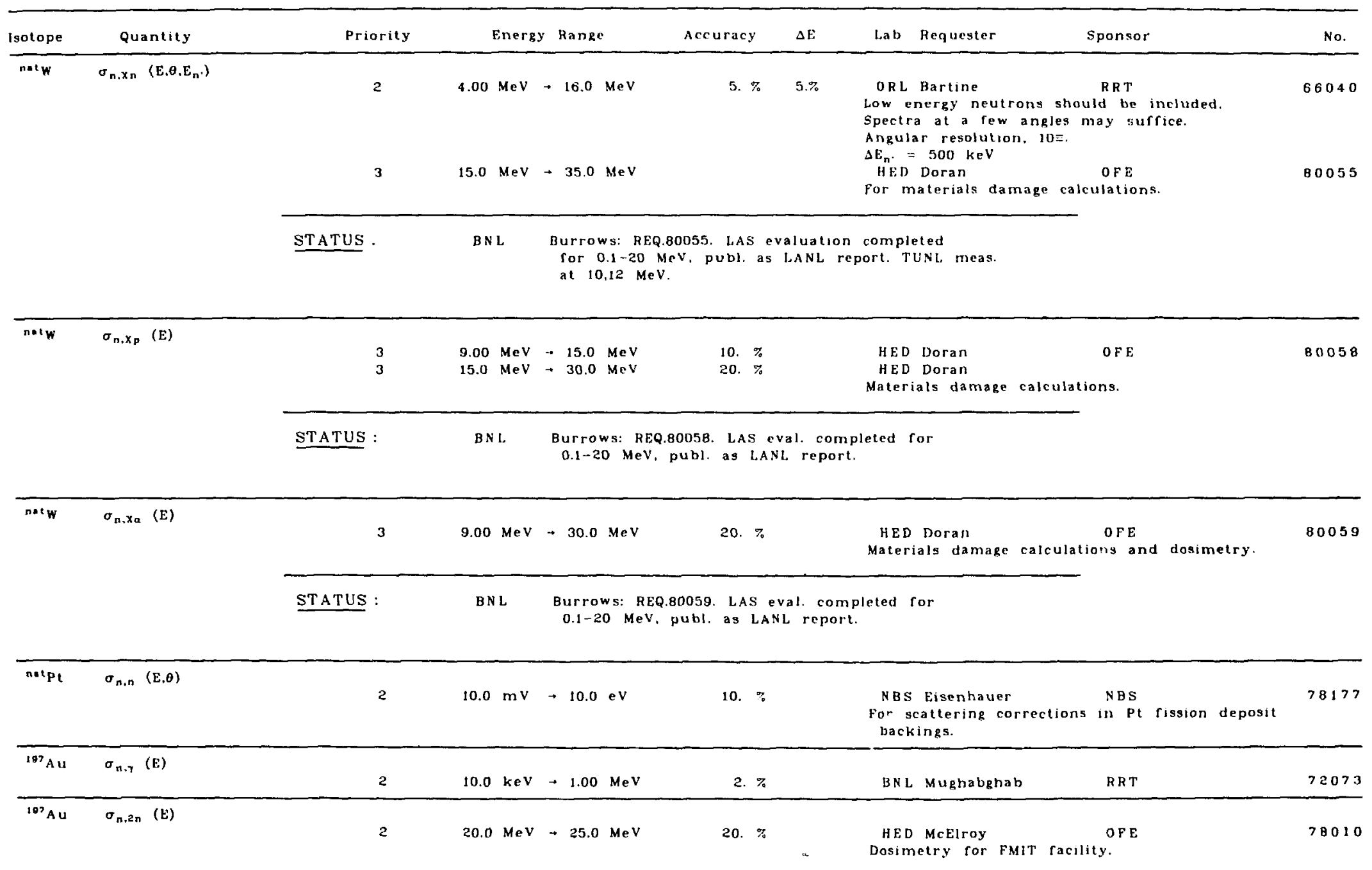


DOE-NDC Compilation of Requests for Nuclear Data 1982

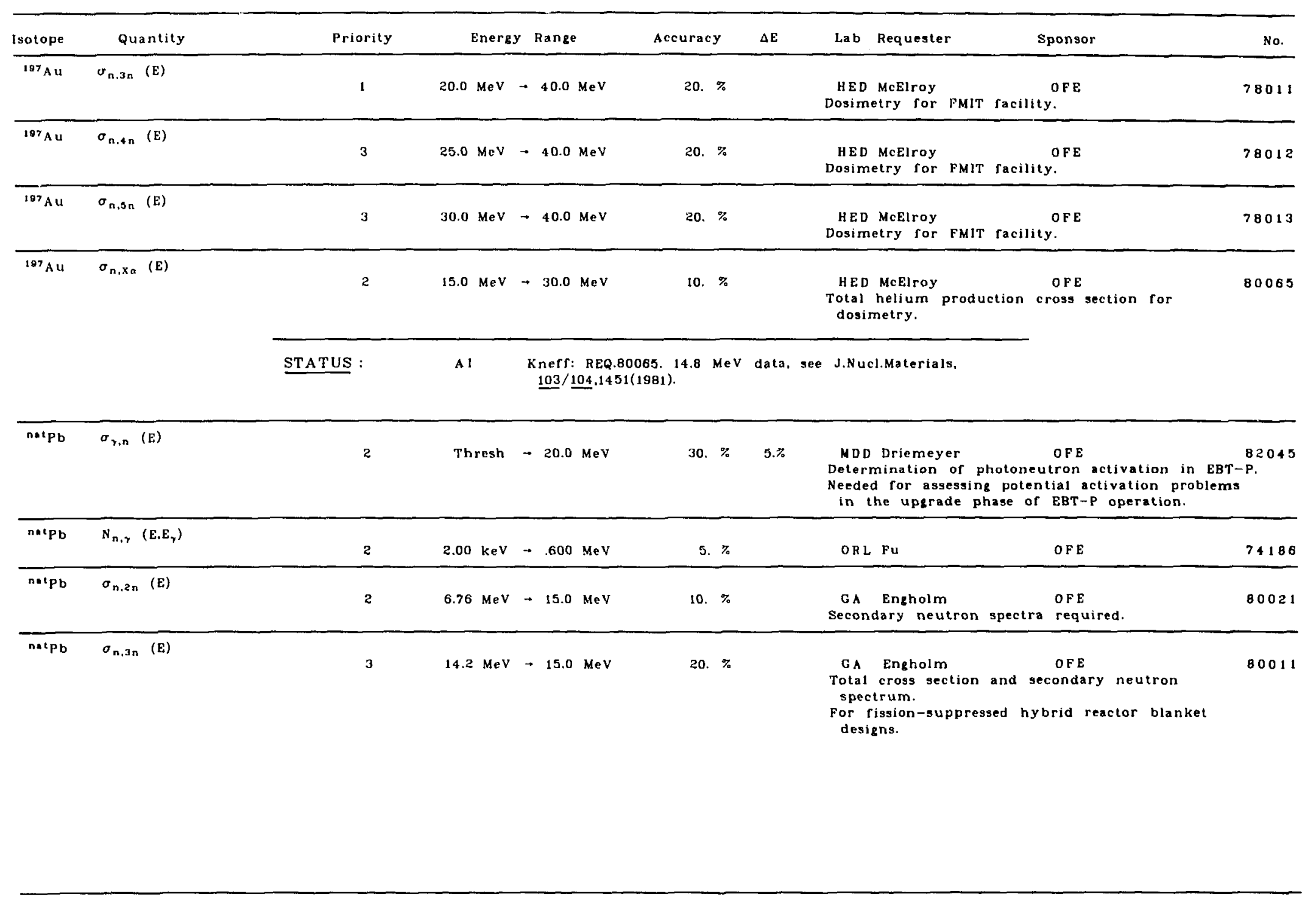


DOE-NDC Compilation of Requests for Nuclear Data 1982

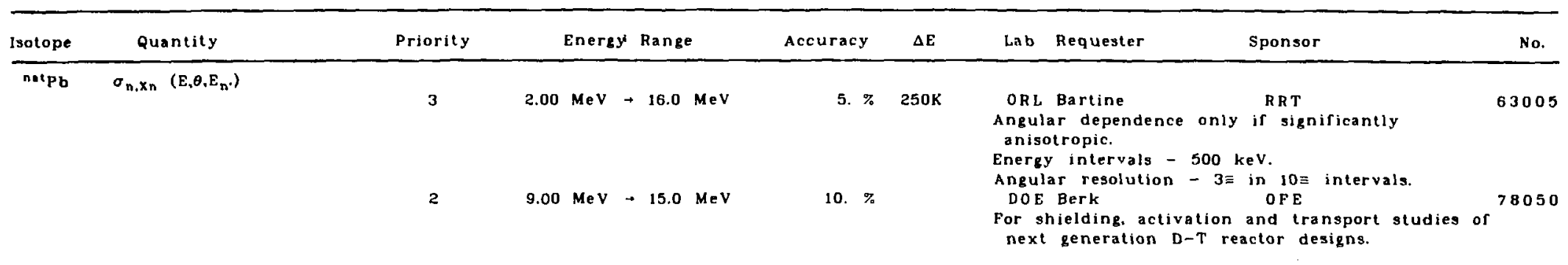

STATUS : $\quad$ BNL Burrows: REQ.78050, TUNL meas. at $10.12 \mathrm{MeV}$

\begin{tabular}{|c|c|c|c|c|c|c|c|c|}
\hline necpb & $\sigma_{n, x}(E)$ & 2 & $9.00 \mathrm{MeV}$ & $\rightarrow \quad 15.0$ & $\mathrm{MeV}$ & $20 . \%$ & $\begin{array}{l}\text { DOE Berk } 0 F E \\
\text { All significant activation reaction cross } \\
\text { sections. } \\
\text { Data needed for shielding. activation and neutron } \\
\text { transport calculations. }\end{array}$ & 80045 \\
\hline${ }^{200} \mathrm{Bi}$ & $\sigma_{n, 2 n}(E)$ & 2 & Thresh & -15.0 & $\mathrm{MeV}$ & $10 . \%$ & $\begin{array}{l}\text { WEW Green } \\
\text { Total (n,2n) cross section and secondary neutron } \\
\text { energy distribution needed. For fission } \\
\text { suppressed hybrid blanket design. }\end{array}$ & 82041 \\
\hline${ }^{230} \mathrm{Th}$ & $\sigma_{n, Y}(E)$ & 2 & $\begin{array}{l}\text { RADIOACTIV } \\
\text { Therma! }\end{array}$ & $\begin{array}{ll}V E & 8.0 \\
\rightarrow & 1.00\end{array}$ & $x\left(10^{* * 4}\right)$ & Y R & $\begin{array}{l}\text { ORL Bartine } \\
\text { Key reaction for production of } U^{232} \text {. }\end{array}$ & 78196 \\
\hline${ }^{232} \mathrm{Th}$ & $\sigma_{n, 101}(E)$ & $\begin{array}{l}2 \\
2 \\
2\end{array}$ & $\begin{array}{l}.100 \mathrm{eV} \\
.100 \mathrm{eV} \\
1.00 \mathrm{keV}\end{array}$ & $\begin{array}{l}\rightarrow \quad 6.00 \\
+\quad 20.0 \\
+\quad 100\end{array}$ & $\begin{array}{l}\text { eV } \\
\text { MeV }\end{array}$ & $\begin{array}{l}.5 \% \\
.5 \% \\
2 . \%\end{array}$ & $\begin{array}{l}\text { BNW Leonard } \\
\text { Needed ror thermal cross section evaluation. } \\
\text { BET Dei } \\
\text { ORL Peelle } \\
\text { Resolved neutron widths needed to } 3-5 \% \\
\text { correlated uncertainties. } \\
\text { For resonance parameter evaluation, several } \\
\text { samples required. }\end{array}$ & $\begin{array}{l}76080 \\
78181 \\
78197\end{array}$ \\
\hline & & STATUS: & ORL & $\begin{array}{r}\text { (REQS } \\
\text { DeSau } \\
\text { (1980 } \\
\text { abov }\end{array}$ & $\begin{array}{l}\text { TS } 76080 \\
\text { 15sure: L } \\
\text { OADV.IN } \\
\text { le } 1.0 \text { e }\end{array}$ & $\begin{array}{l}, 78181 \text { ) } \\
\text { ittle (ANS,32, } \\
\text { REACT.PHYS } \\
\text { RACT }\end{array}$ & $\begin{array}{l}\text { d Olsen } \\
\text { satisfy this }\end{array}$ & \\
\hline
\end{tabular}


DOE-NDC Compilation of Requests for Nuclear Data 1982

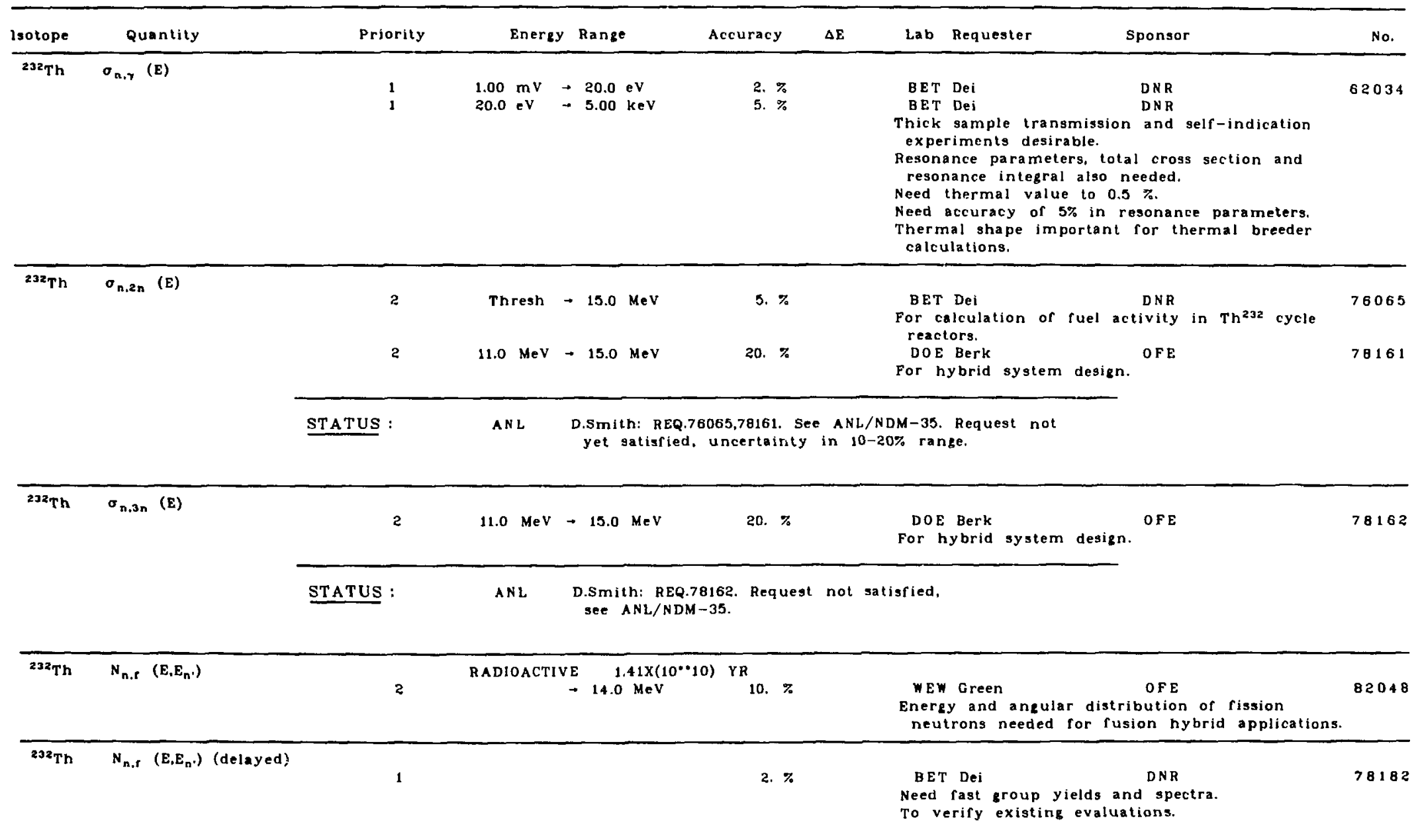


DOE-NDC Compilation of Requests for Nuclear Data 1982

\begin{tabular}{|c|c|c|c|c|c|c|c|c|}
\hline Isotope & Quantity & Priority & Energy & Range & Accuracy & $\Delta \mathrm{E}$ & Lab Requester & No. \\
\hline${ }^{231} \mathbf{P a}$ & $\sigma_{n, \gamma}(E)$ & $\begin{array}{l}2 \\
2\end{array}$ & $\begin{array}{l}\text { RADIOACTIVE } \\
\text { Thermal } \rightarrow \\
1.00 \mathrm{mV} \rightarrow\end{array}$ & $\begin{array}{l}\quad 3.28 \times\left(10^{-4}\right) \\
+10.0 \mathrm{MeV} \\
+1.00 \mathrm{keV} \quad 5\end{array}$ & $\begin{array}{l}\text { YR } 10 . \% \\
5 . \rightarrow 10 . \%\end{array}$ & & $\begin{array}{l}\text { BNW Leonard } \\
\text { For control of } \mathrm{U}^{232} \text { production. } \\
\text { BET Dei } \\
\text { Also need resonance parameters and resonance } \\
\text { integral. } \\
\text { For calculation of fuel activity in } \mathrm{Th}^{232} \text { cycle } \\
\text { reactors. }\end{array}$ & $\begin{array}{l}69219 \\
76066\end{array}$ \\
\hline${ }^{231} \mathrm{~Pa}$ & $N_{n, f}\left(E, E_{n}\right)$ (delayed) & 2 & RADIOACTIVE & E $\quad 3.28 \times\left(10^{* .4}\right)$ & 5. \% & & $\begin{array}{l}\text { BET Dei } \\
\text { Need fast group yields and spectra. } \\
\text { To verify existing evaluations. }\end{array}$ & 78183 \\
\hline${ }^{233} \mathrm{~Pa}$ & $\sigma_{n, 7}(E)$ & 2 & $\begin{array}{l}\text { RADIOACTIVE } \\
1.00 \mathrm{mV} \rightarrow\end{array}$ & $\begin{array}{l}\text { E } 27.0 \mathrm{DAY} \\
+\quad .100 \mathrm{keV}\end{array}$ & 5. $\%$ & & $\begin{array}{l}\text { BET Dei } \\
\text { Resonance parameters also desired. } \\
\text { Needed for analygis of } \mathrm{Th}^{232} \text { cycle thermal } \\
\text { reactors. }\end{array}$ & 76059 \\
\hline${ }^{202} \mathrm{U}$ & $\sigma_{n, \gamma}(E)$ & 2 & $\begin{array}{l}\text { RADIOACTIVE } \\
1.00 \mathrm{mV}\end{array}$ & $\begin{array}{lrl}E & 72 & \text { YR } \\
& 1.00 & \mathrm{keV}\end{array}$ & 2. $\rightarrow 5 . \%$ & & $\begin{array}{l}\text { BET Del } \\
\text { Also need resonance parameters and resonance } \\
\text { integral. } \\
\text { For calculation of fuel activity in } \mathrm{Th}^{232} \text { cycle } \\
\text { reactors. }\end{array}$ & 76067 \\
\hline${ }^{233} \mathrm{U}$ & Prompt $\bar{v}$ & 2 & $\begin{array}{l}\text { RADIOACTIVE } \\
14.0 \mathrm{MeV}\end{array}$ & E $\quad 1.592 \times\left(10^{* .5}\right)$ & $\begin{array}{lll}Y R & \\
& \text { 2. } \%\end{array}$ & & $\begin{array}{l}\text { LAS Young } \\
\text { Prompt } \bar{v} \text { wanted. } \\
\text { Needed for multipurpose evaluation. }\end{array}$ & 78164 \\
\hline${ }^{233} \mathrm{U}$ & $\sigma_{n, t a t}(E)$ & 2 & $\begin{array}{l}\text { RADIOACTIVE } \\
1.00 \mathrm{mV} \rightarrow \\
60.0 \mathrm{eV} \rightarrow\end{array}$ & $\begin{array}{l}\mathrm{E} \quad 1.592 \times\left(10^{* * 5}\right) \\
\rightarrow \quad 2.00 \mathrm{eV} \\
+\quad 100 \mathrm{MeV}\end{array}$ & $\begin{array}{l}\text { YR } \begin{array}{l}.5 \% \\
\text { 3. } \%\end{array}\end{array}$ & & $\begin{array}{l}\text { BNW Leonard } \\
\text { Needed for thermal cross section evaluation. } \\
\text { LAS Stewart } \\
\text { Needed to cover the unresolved range and overlap } \\
\text { the recent ANL data which begins at } 42 \text { keV. }\end{array}$ & $\begin{array}{l}76082 \\
79001\end{array}$ \\
\hline${ }^{233} \mathrm{U}$ & $\sigma_{n, n^{\prime}}\left(E, E_{n}\right)$ & 2 & $\begin{array}{l}\text { RADIOACTIVE } \\
40.0 \mathrm{keV} \rightarrow\end{array}$ & $\begin{array}{l}\text { E } \quad 1.592 \times\left(10^{* * 5}\right) \\
\rightarrow \quad 7.00 \mathrm{MeV} 10\end{array}$ & 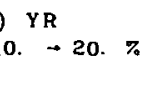 & & $\begin{array}{l}\text { ANL Smith } \\
\text { Accuracy of } 5-10 \% \text { above } 0.5 \mathrm{MeV} \text {. }\end{array}$ & 67086 \\
\hline
\end{tabular}




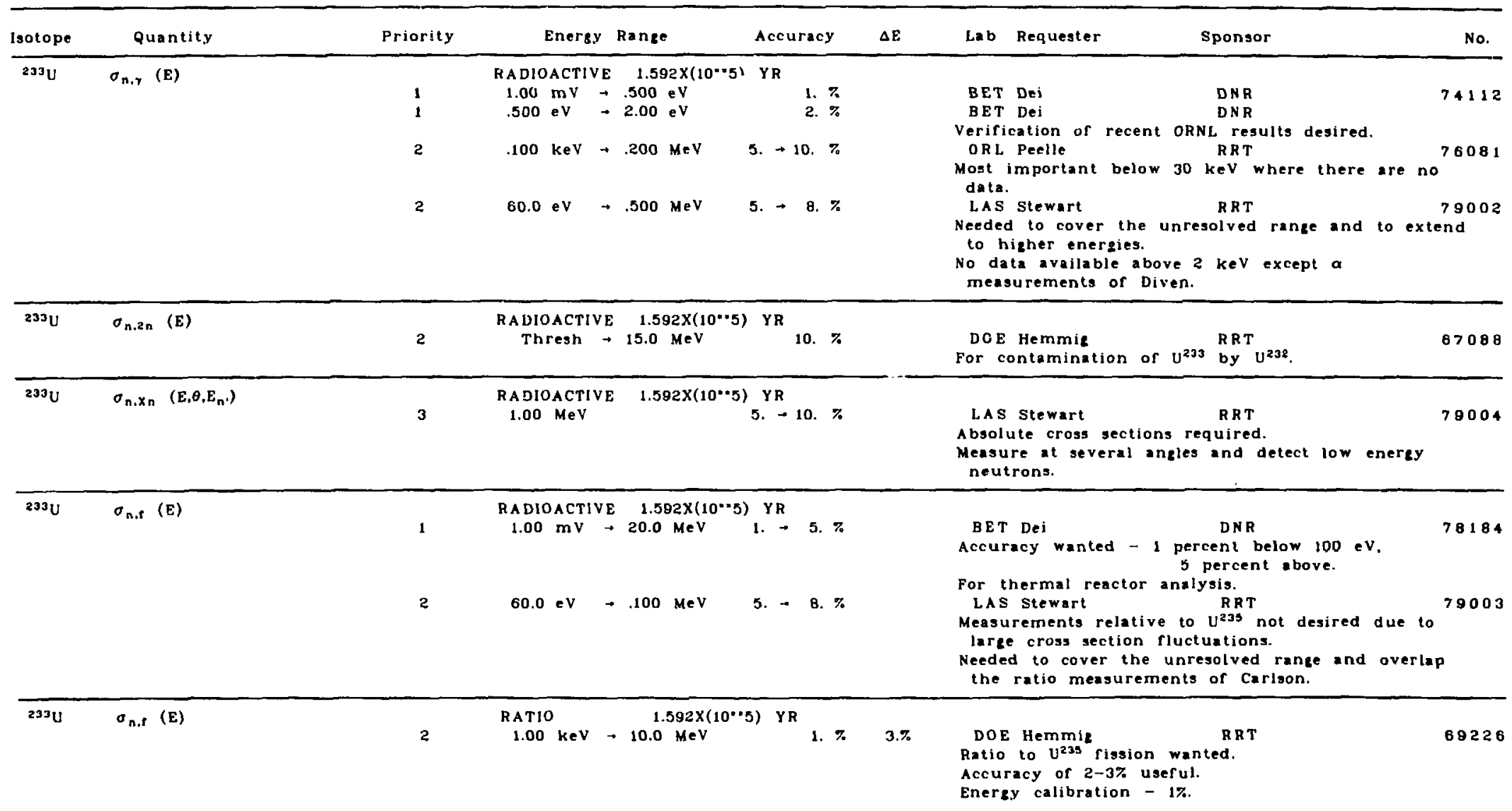


DOE-NDC Compilation of Requests for Nuclear Data 1982

\begin{tabular}{|c|c|c|c|c|c|c|c|c|c|}
\hline Isotope & Quantity & Priority & & Enerey & Range & Accuracy & $\Delta E$ & Lab Requester & No. \\
\hline${ }^{223} \mathrm{U}$ & Alpha (E) & $\begin{array}{l}1 \\
2 \\
2\end{array}$ & $\begin{array}{l}\text { R A D IO } \\
5.00 \\
.500 \\
10.0\end{array}$ & $\begin{array}{l}\mathrm{ACTIVE} \\
\mathrm{mV} \rightarrow \\
\mathrm{eV} \rightarrow \\
\mathrm{keV} \rightarrow\end{array}$ & $\begin{array}{l}1.592 \times\left(10^{* * 5)}\right. \\
.500 \mathrm{eV} \\
10.0 \mathrm{keV} \\
20.0 \mathrm{MeV}\end{array}$ & $\begin{array}{r}\text { YR } \\
2 . \rightarrow \begin{array}{r}\text { 8. } \% \\
\text { 3. \% } \\
5 . \rightarrow 10 . \%\end{array}\end{array}$ & & $\begin{array}{l}\text { BET Dei } \\
\text { BET Dei } \\
\text { BET Dei } \\
\text { DNR } \\
\text { Capture cross section equally useful. } \\
\text { integral experiments needed to resolve } \\
\text { discrepancies. } \\
\text { Want } \eta \text { to } 0.25 \% \text { below } 3 \mathrm{eV}(1 \% \text { useful below } \\
1 \text { eV). } 1 \% \text { from } 30 \text { eV } 10 \mathrm{keV}(5 \% \text { useful). } \\
\text { Want } \eta 2 \% \text { trom } 1-30 \mathrm{keV} \text {. } \\
\text { Want verification of recent oRNL and Bettis work. }\end{array}$ & 62041 \\
\hline${ }^{233} \mathrm{U}$ & Alpha (E) & $\begin{array}{l}2 \\
2\end{array}$ & $\begin{array}{l}\text { RADIO } \\
1.00 \\
1.00\end{array}$ & $\begin{array}{l}\text { ACTIVE } \\
\mathrm{keV} \rightarrow \\
\mathrm{keV} \rightarrow\end{array}$ & $\begin{array}{l}1.592 \times\left(10^{*} \cdot 5\right) \\
+3.00 \mathrm{MeV} \quad 10 \\
3.00 \mathrm{MeV} \quad 10\end{array}$ & 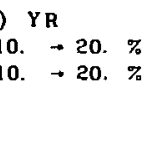 & & $\begin{array}{lc}\text { ANL Smith } & \text { RRT } \\
\text { DOE Hemmig } & \text { RRT } \\
\text { Capture cross section equally userul. } \\
\text { Want } \eta \text { to } 2 \% \text { from 1-30 keV. }\end{array}$ & 67090 \\
\hline${ }^{233} \mathrm{U}$ & Eta (E) & 1 & $\begin{array}{l}\text { RADIO } \\
1.00\end{array}$ & $\begin{array}{l}\text { OACTIVE } \\
\mathrm{mV}\end{array}$ & $\begin{array}{l}\text { E } \quad 1.592 \times\left(10^{*} 5\right) \\
1.00 \mathrm{eV}\end{array}$ & $\begin{array}{ll}\mathrm{YR} \\
.4 \%\end{array}$ & & $\begin{array}{l}\text { BET Dei } \\
\text { Thermal value and shape needed. } \\
\text { To verify manganese bath regulls. }\end{array}$ & 74113 \\
\hline${ }^{233} \mathrm{U}$ & $\bar{\nu}(E)$ & $\begin{array}{l}1 \\
1 \\
1\end{array}$ & $\begin{array}{r}\text { RADIC } \\
1.00 \\
30.0 \\
1.00\end{array}$ & $\begin{array}{l}\text { OACTIVE } \\
\text { mV } \rightarrow \\
\text { eV } \\
\text { keV } \rightarrow\end{array}$ & $\begin{array}{l}1.592 \times(10 * 5) \\
30.0 \mathrm{eV} \\
+1.00 \mathrm{keV} \\
10.0 \mathrm{keV}\end{array}$ & $\begin{array}{l}\text { YR } \\
\begin{aligned} .25 \% \\
1 . \% \\
\text { 2. } \%\end{aligned}\end{array}$ & & $\begin{array}{lc}\text { BET Dei } & \text { DNR } \\
\text { BET Dei } & \text { DNR } \\
\text { BET Dei } & \text { DNR } \\
\text { Measurement relative to } \mathrm{U}^{235} \text { and } \mathrm{Pu}^{230} \\
\text { preferred. } \\
\text { Low energy structure may be important. } \\
\text { Needed to resolve discrepancies in thermal } \\
\text { parameters and breeding prediction. }\end{array}$ & 69443 \\
\hline${ }^{233} \mathrm{U}$ & $\bar{\nu}$ (E) (delayed) & 1 & $\begin{array}{l}\text { RADIC } \\
\text { Th }\end{array}$ & $\begin{array}{l}\text { DACTIVE } \\
\text { erma! }\end{array}$ & $1.592 \times\left(10^{\circ .5}\right)$ & $\begin{array}{ll} \\
Y R & \\
& 5 . \%\end{array}$ & & $\begin{array}{ll}\text { BET Del } & \text { DNR } \\
\text { To resolve discrepancies. } & \end{array}$ & 74116 \\
\hline $233 \mathrm{U}$ & $N_{n, r}\left(E, E_{n}\right)$ & 1 & $\begin{array}{l}\text { RADIC } \\
\text { Th }\end{array}$ & $\begin{array}{l}\text { Dactive } \\
\text { ermal }\end{array}$ & $1.592 \times\left(10^{* .5}\right)$ & $\begin{array}{lll}Y R & \\
& 1 . \%\end{array}$ & & $\begin{array}{l}\text { BET Dei } \\
\text { Need shape of neutron energy distribution from } \\
100 \text { keV } 1015 \text { MeV. } \\
\text { Relative peak to } 1 \% \text {. } \\
\text { Needed ror criticality calculations. }\end{array}$ & 78185 \\
\hline
\end{tabular}


DOE-NDC Compilation of Requests for Nuclear Data 1982

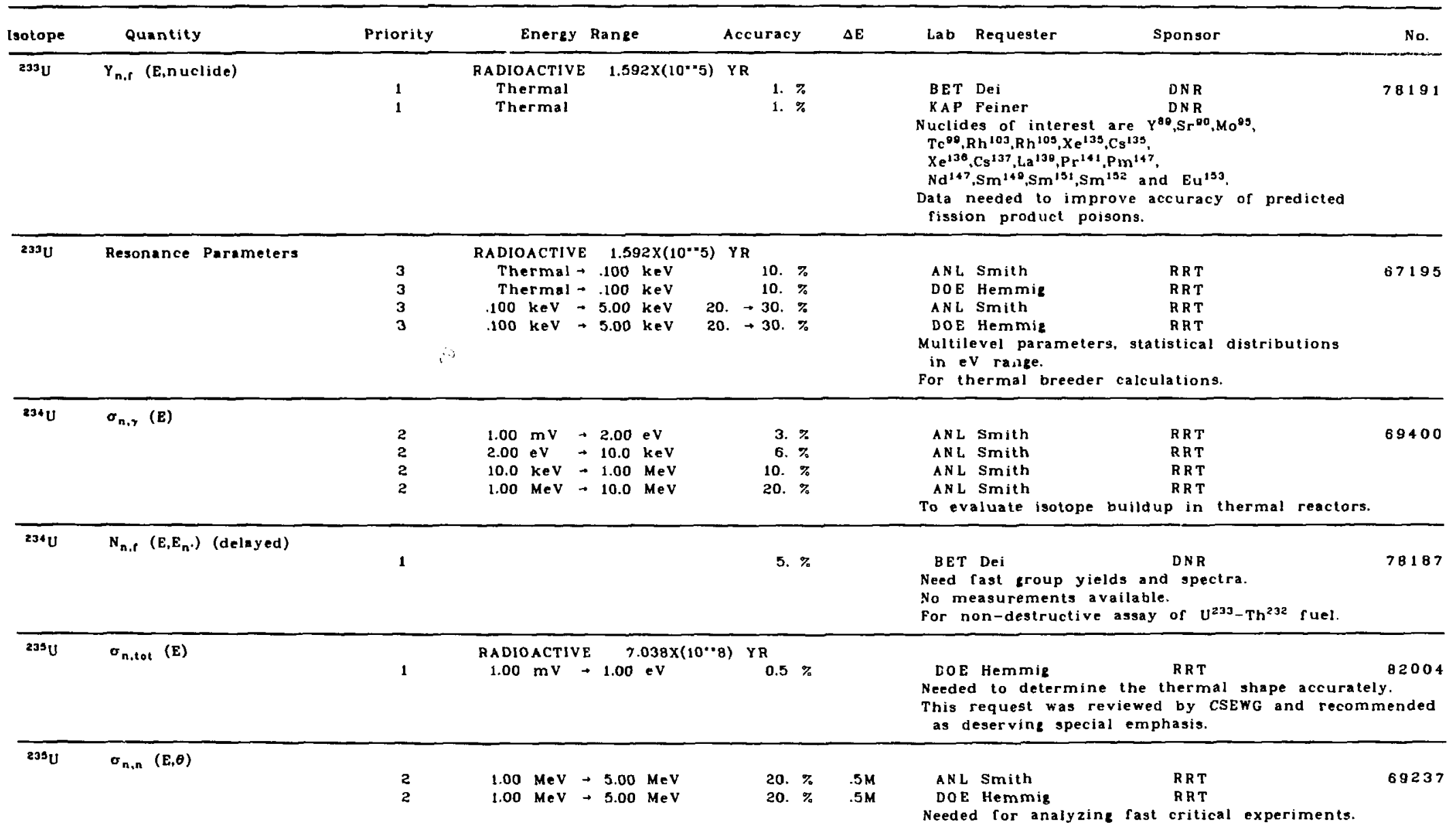




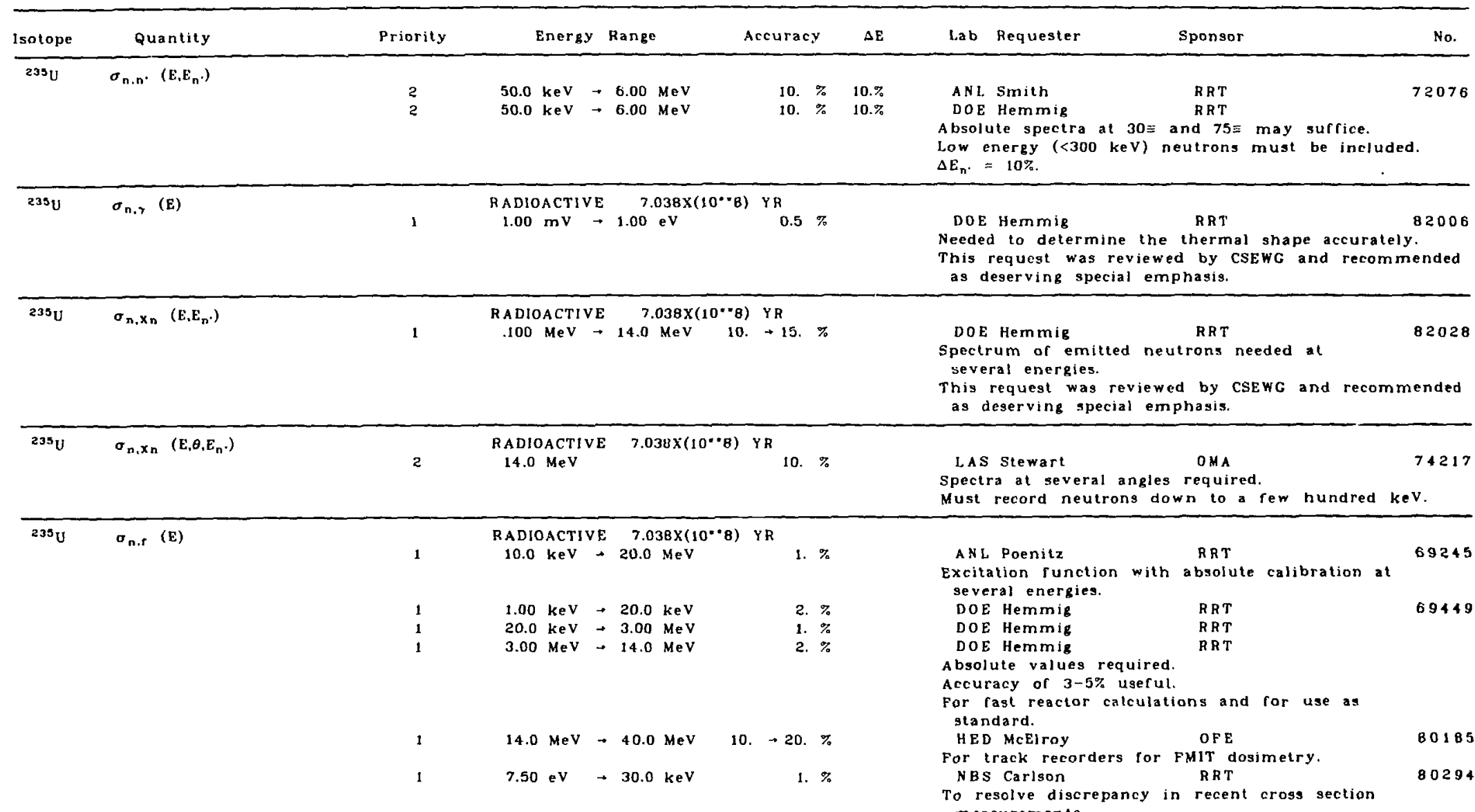


DOE-NDC Compilation of Requests for Nuclear Data 1982

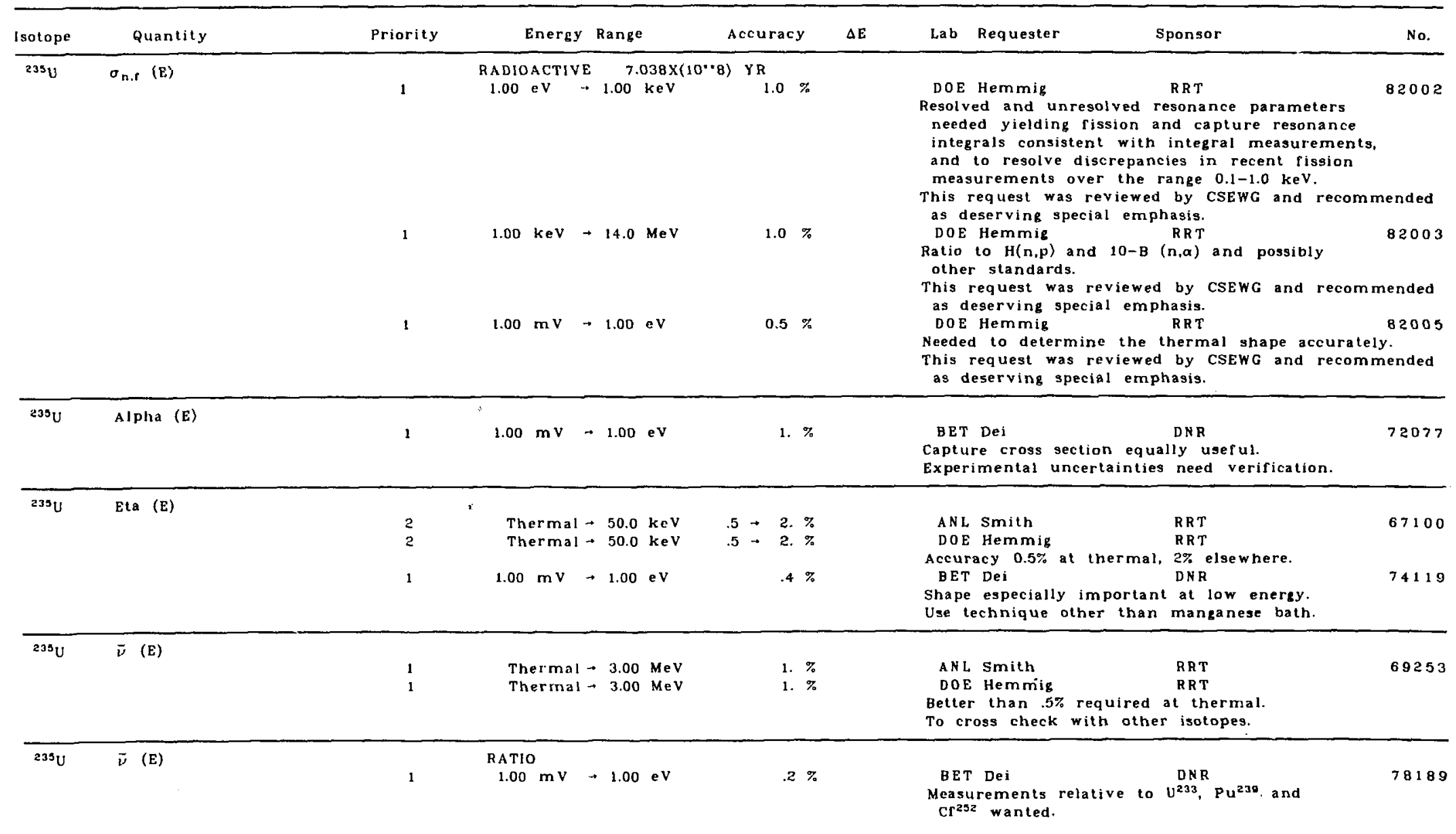




\begin{tabular}{|c|c|c|c|c|c|c|c|c|}
\hline Isotope & Quantity & Priority & Energy & Range & Accuracy & $\Delta \mathbf{E}$ & Lab Requester & No. \\
\hline${ }^{233} \mathrm{U}$ & $\bar{v}(E)$ (delayed) & 1 & & & 3. $\%$ & & $\begin{array}{l}\text { BET Dei } \\
\text { For the entire energy range. } \\
\text { To resolve uncertainties in available data. }\end{array}$ & 74120 \\
\hline${ }^{235} \mathrm{U}$ & $N_{n, f}\left(E, E_{n^{\prime}}\right)$ & $\begin{array}{l}2 \\
2 \\
1\end{array}$ & $\begin{array}{l}\text { Thermal } \rightarrow \\
\text { Thermal } \rightarrow \\
\text { Thermal }\end{array}$ & $\begin{array}{l}3.00 \mathrm{MeV} \\
3.00 \mathrm{MeV}\end{array}$ & $\begin{array}{l}\text { 5. } \% \\
\text { 5. } \% \\
\text { 1. } \%\end{array}$ & & $\begin{array}{l}\text { ANL Smith } \\
\text { DOE Hemmig } \\
\text { Rerification of fission spectrum. } \\
\text { BET Dei } \\
\text { Need shape or neutron energy distribution from } \\
100 \text { keV to } 15 \text { MeV. } \\
\text { Relalive peak to } 1 \% \text {. } \\
\text { Needed ror criticality calculations. }\end{array}$ & $\begin{array}{l}69256 \\
72080\end{array}$ \\
\hline${ }^{235} \mathrm{U}$ & $N_{n, f}\left(E, E_{n^{\prime}}\right) \quad$ (delayed) & 2 & Thermal - & $5.00 \mathrm{MeV}$ & 5. \% & & $\begin{array}{l}\text { DOE Hemmig } \\
\text { Yield, half-lire and energy needed. } \\
\text { For analysis of fast criticals and to check } \\
\text { existing data. }\end{array}$ & 69260 \\
\hline${ }^{235} \mathrm{U}$ & $N_{n, f}\left(E, E_{\gamma}\right)$ (delayed) & 3 & Thermal & & 15. $\%$ & & $\begin{array}{l}\text { LAS Walton } \\
\text { Spectra } 0.25-5 \mathrm{MeV} \text { and time-dependent yield } \\
1 \text { msec-12 hr. } \\
\text { Associate gammas with fission products. } \\
\text { Ge(Li) resolution - } 2.5 \mathrm{keV} \text { at } 1.2 \mathrm{MeV} \text {. } \\
\text { For non-destructive assays of } \mathrm{U}^{235} \text {. }\end{array}$ & 70029 \\
\hline${ }^{235} \mathrm{U}$ & $Y_{\text {n.r }}($ E,nuclide) & $\begin{array}{l}1 \\
1\end{array}$ & $\begin{array}{l}\text { Thermal } \\
\text { Thermal }\end{array}$ & & $\begin{array}{l}\text { 1. } \% \\
\text { 1. } \%\end{array}$ & & 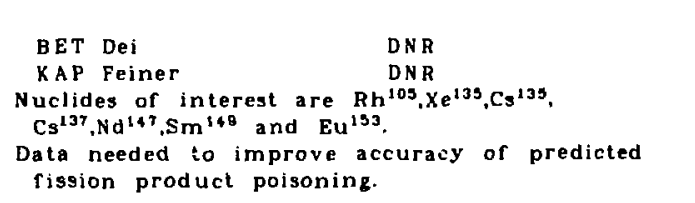 & 78192 \\
\hline${ }^{235} \mathrm{U}$ & Resonance Parameters & 1 & $\begin{array}{l}\text { Thermal } \rightarrow \\
\text { Thermal } \rightarrow \\
\text { Thermal } \rightarrow\end{array}$ & $\begin{array}{l}.200 \mathrm{keV} \\
.200 \mathrm{keV} \\
.200 \mathrm{keV}\end{array}$ & $\begin{array}{l}10 . \% \\
10 . \% \\
10 . \%\end{array}$ & & $\begin{array}{l}\text { ANL Smiin } \\
\text { DOE Hemmig } \\
\text { RRT } \\
\text { Multilevel fit where feasible. } \\
\text { For extrapolation to unresolved resonance region. } \\
\text { BET Dei } \\
\text { Multilevel fit where feasible. } \\
\text { Verification of existing data useful. }\end{array}$ & 69202 \\
\hline
\end{tabular}




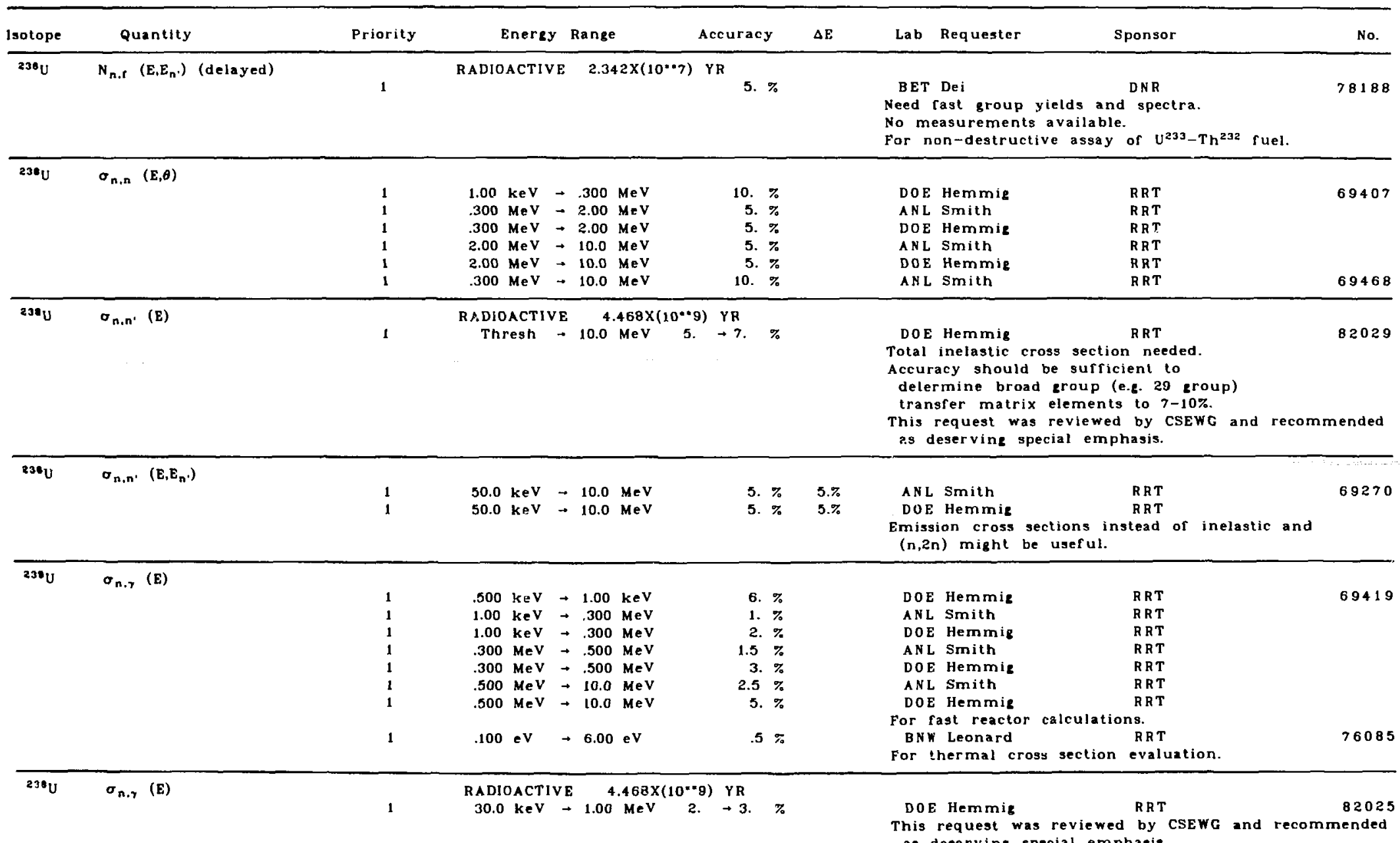

as deservin: special emphasis. 


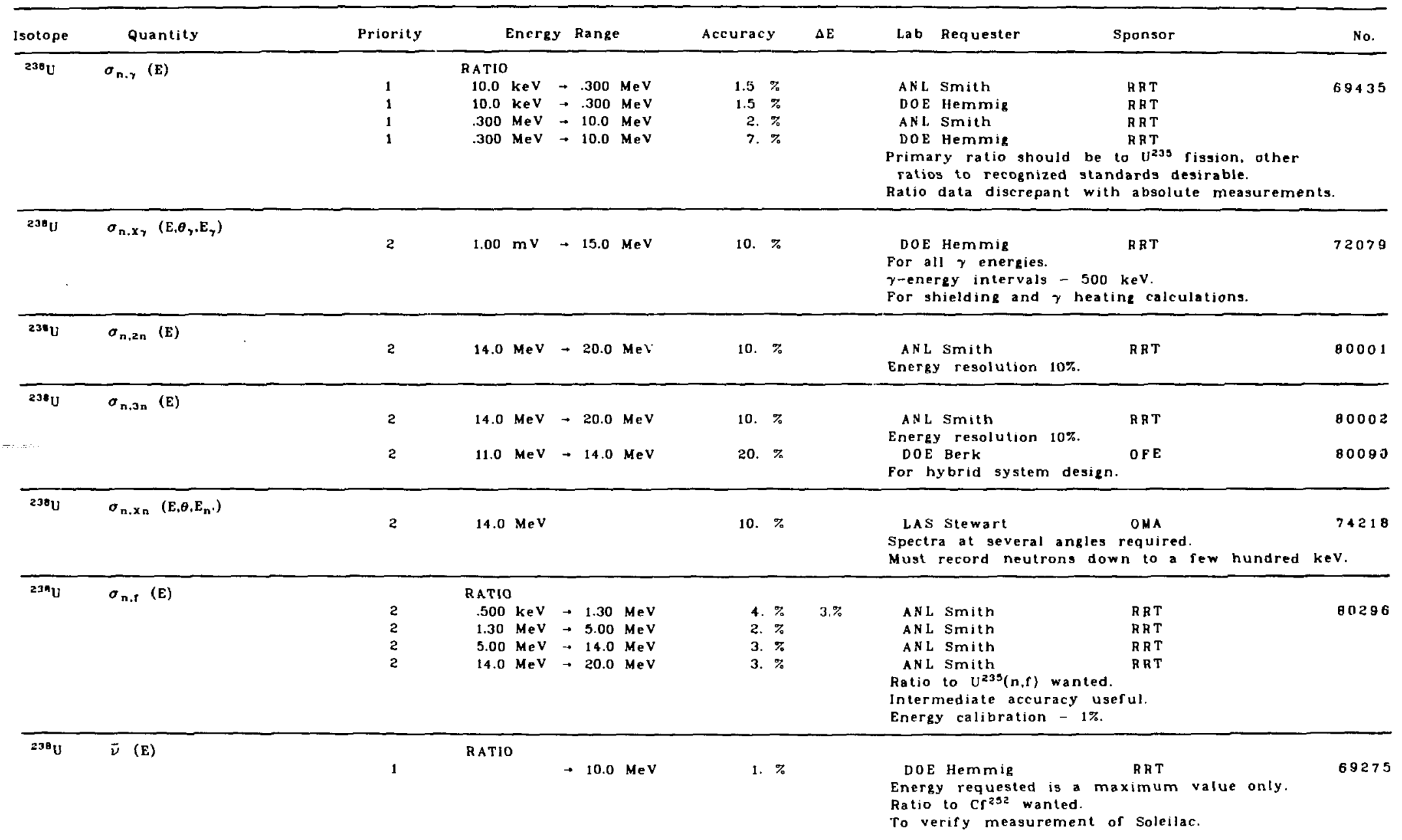


DOE-NDC Compilation of Requests for Nuclear Data 1982

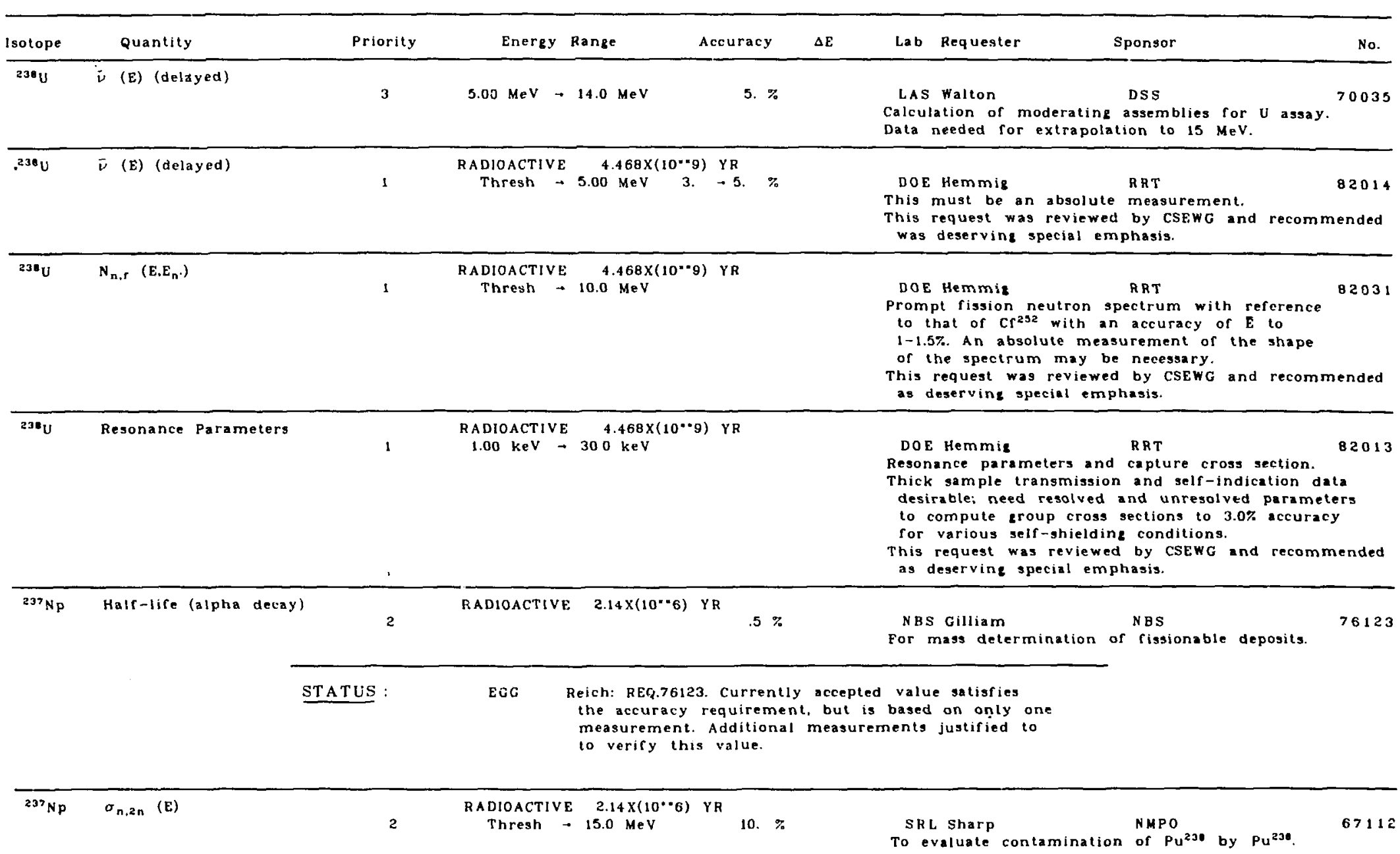


DOE-NDC Compilation of Requests for Nuclear Data 1982

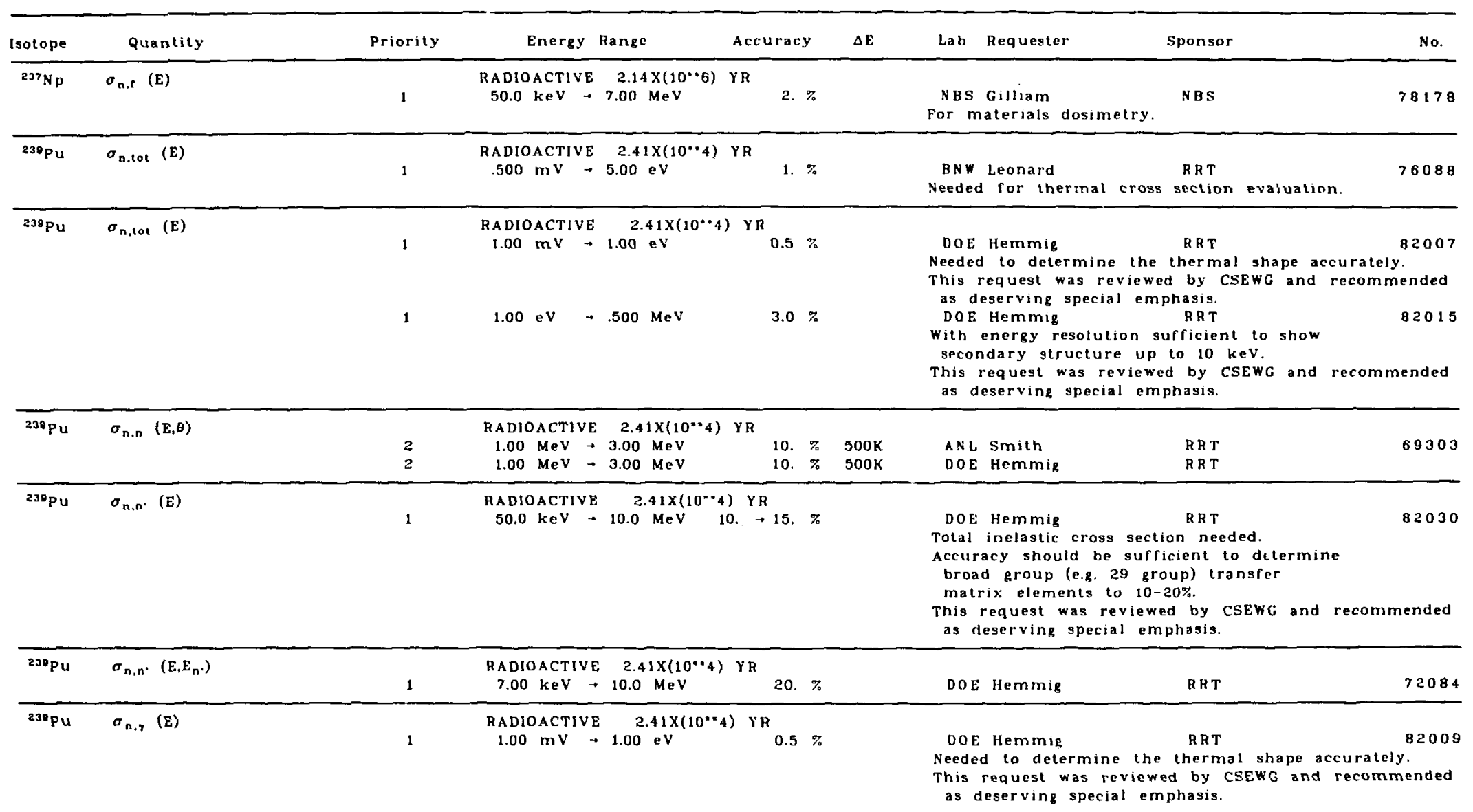


DOE-NDC Compilation of Requests for Nuclear Data

1982

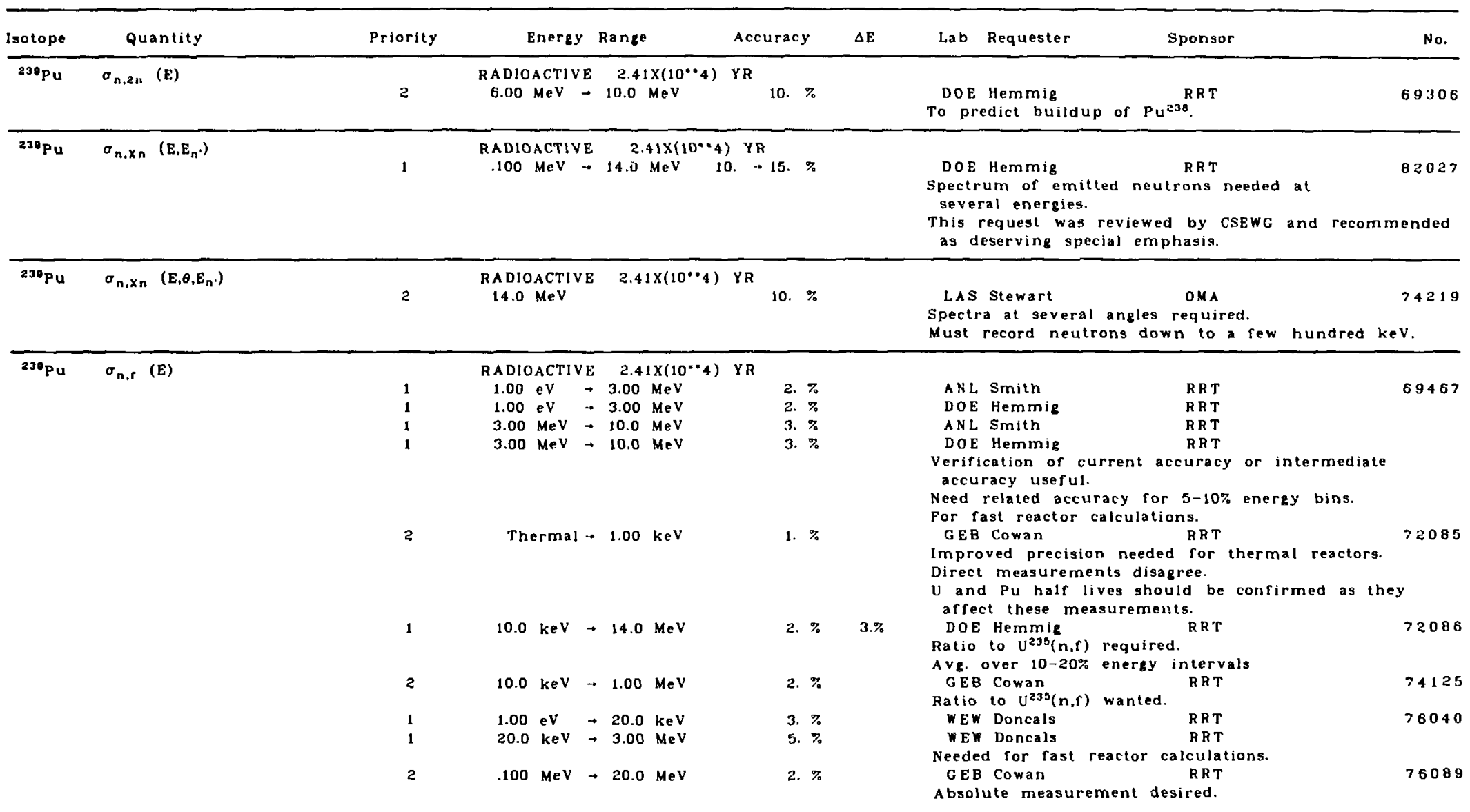


DOE-NDC Compilation of Requests for Nuclear Data 1982

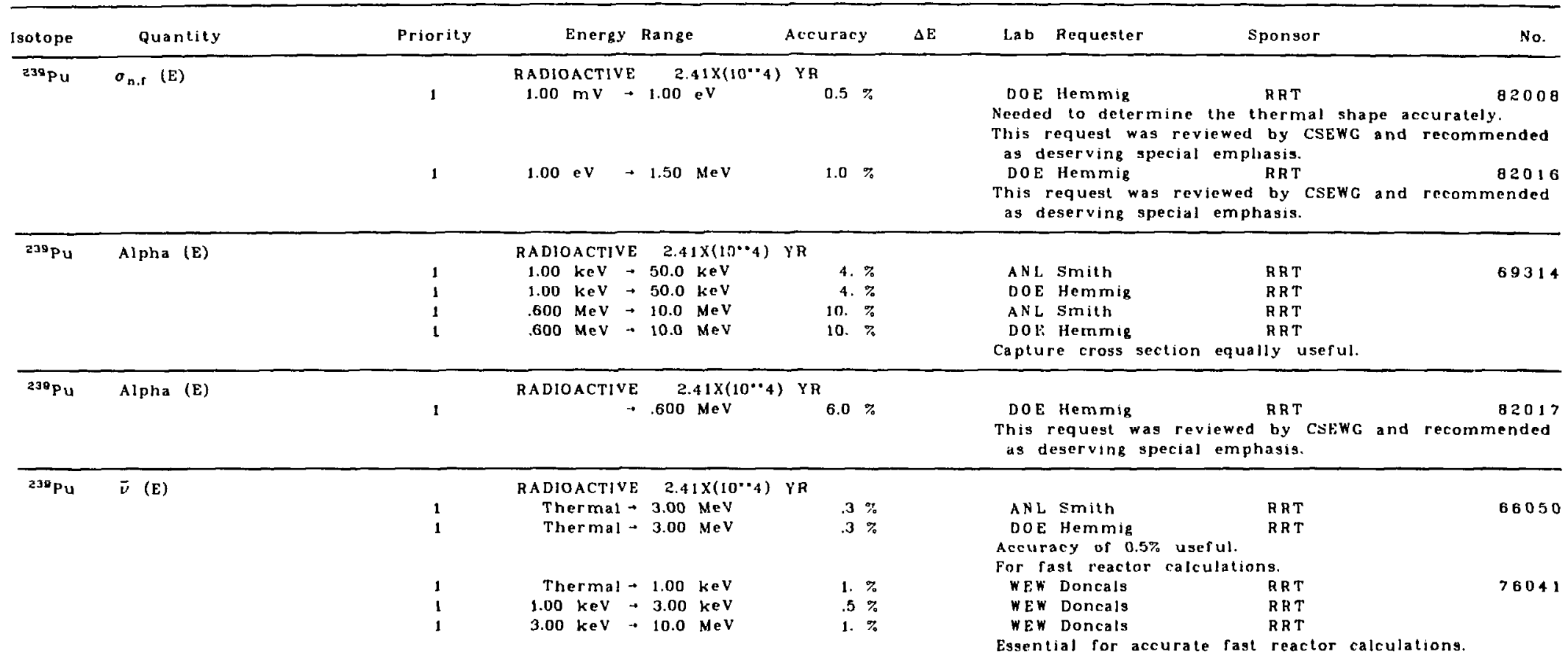

STATUS :
ORL Weston: REQ.66050.76041. Gwin (ORNL) has new
measurements from t keV to $10 \mathrm{MeV}$ to $\approx 1.25 \%$

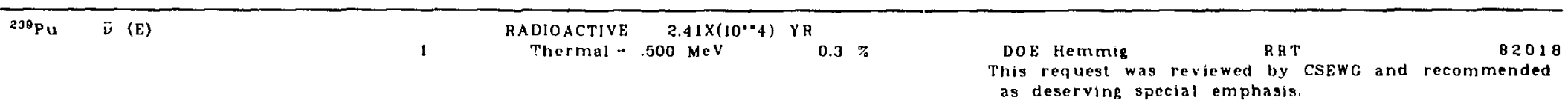


DOE-NDC Compilation of Requests for Nuclear Data 1982

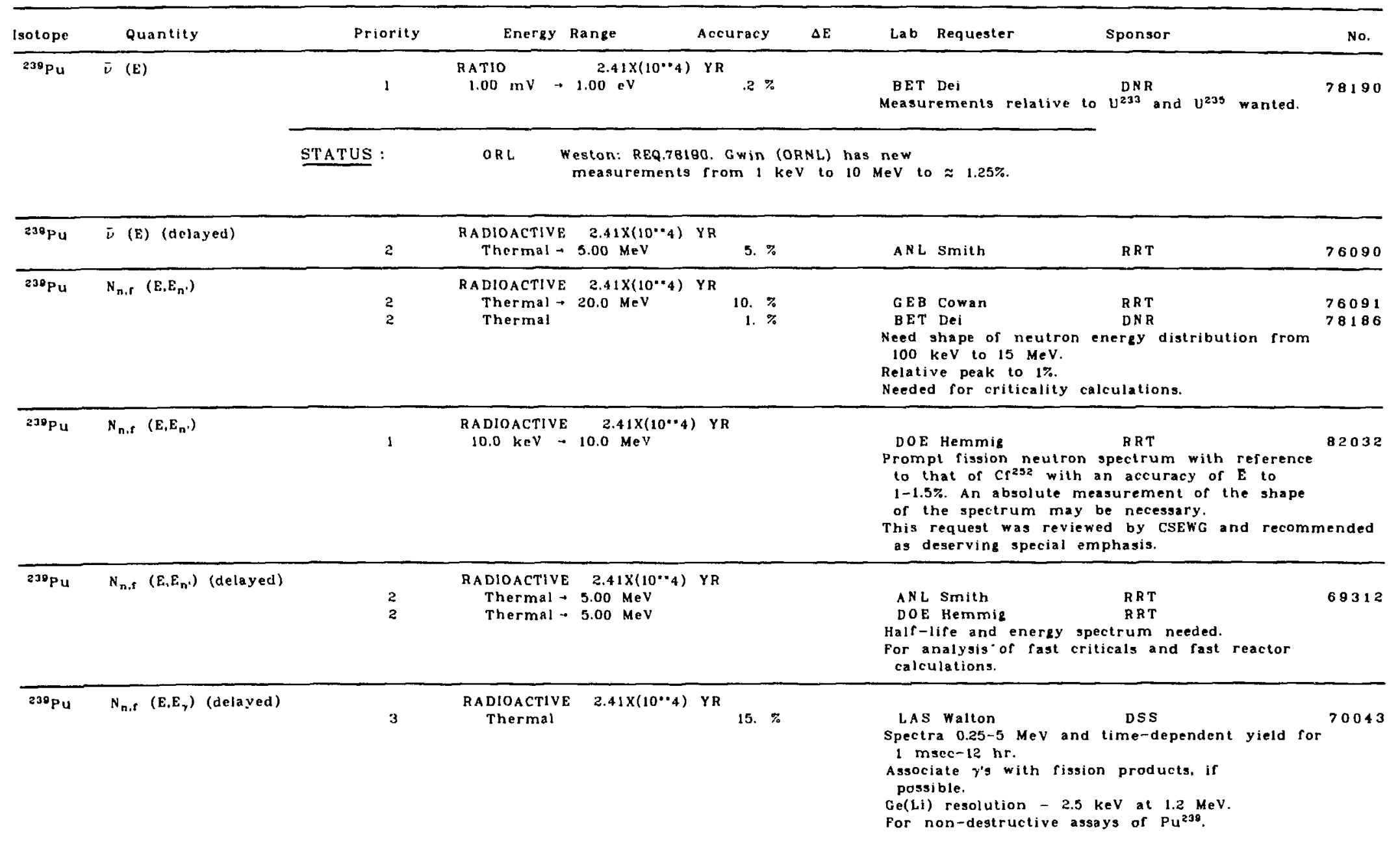


DOE-NDC Compilation of Requests for Nuclear Data 1982

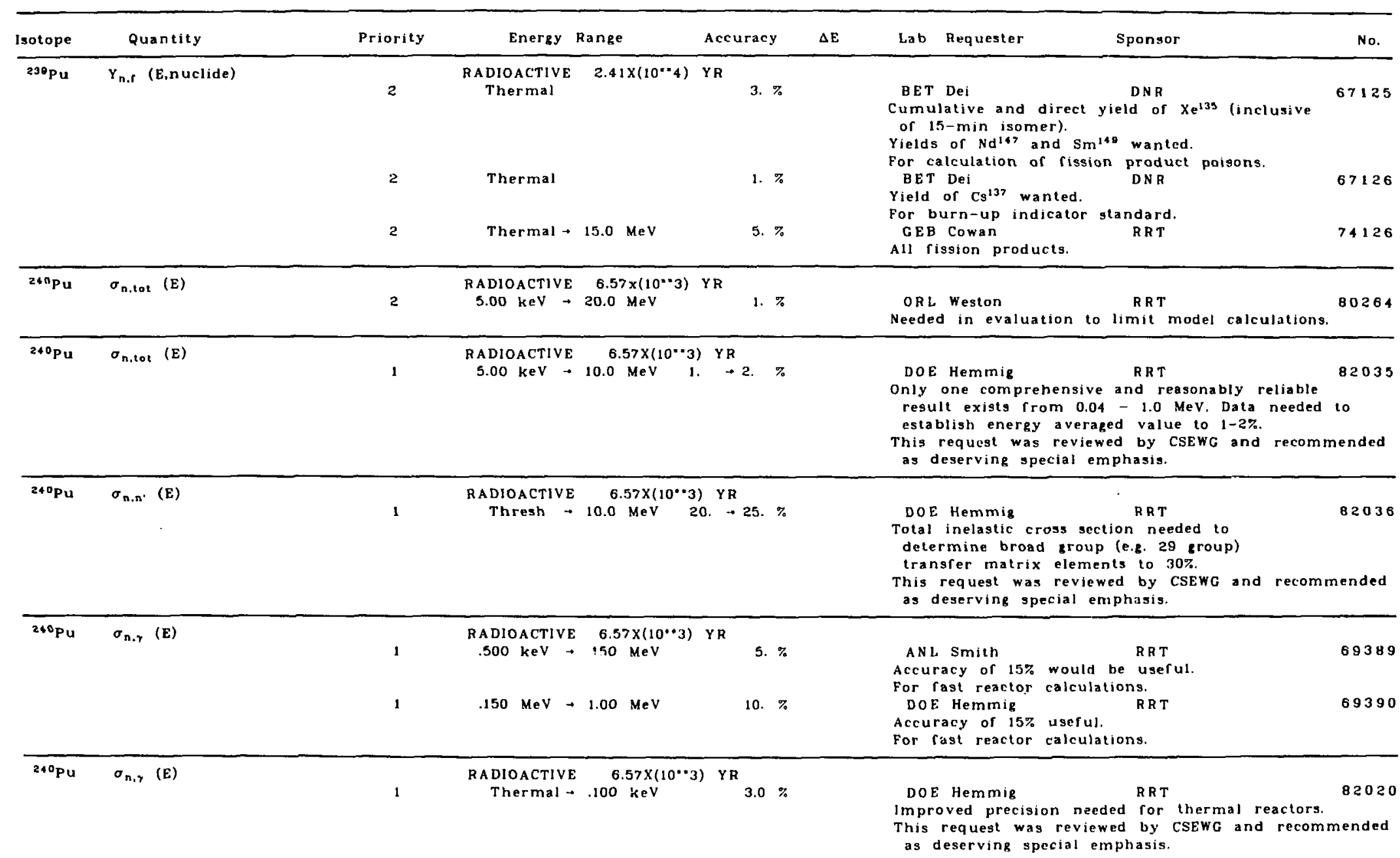




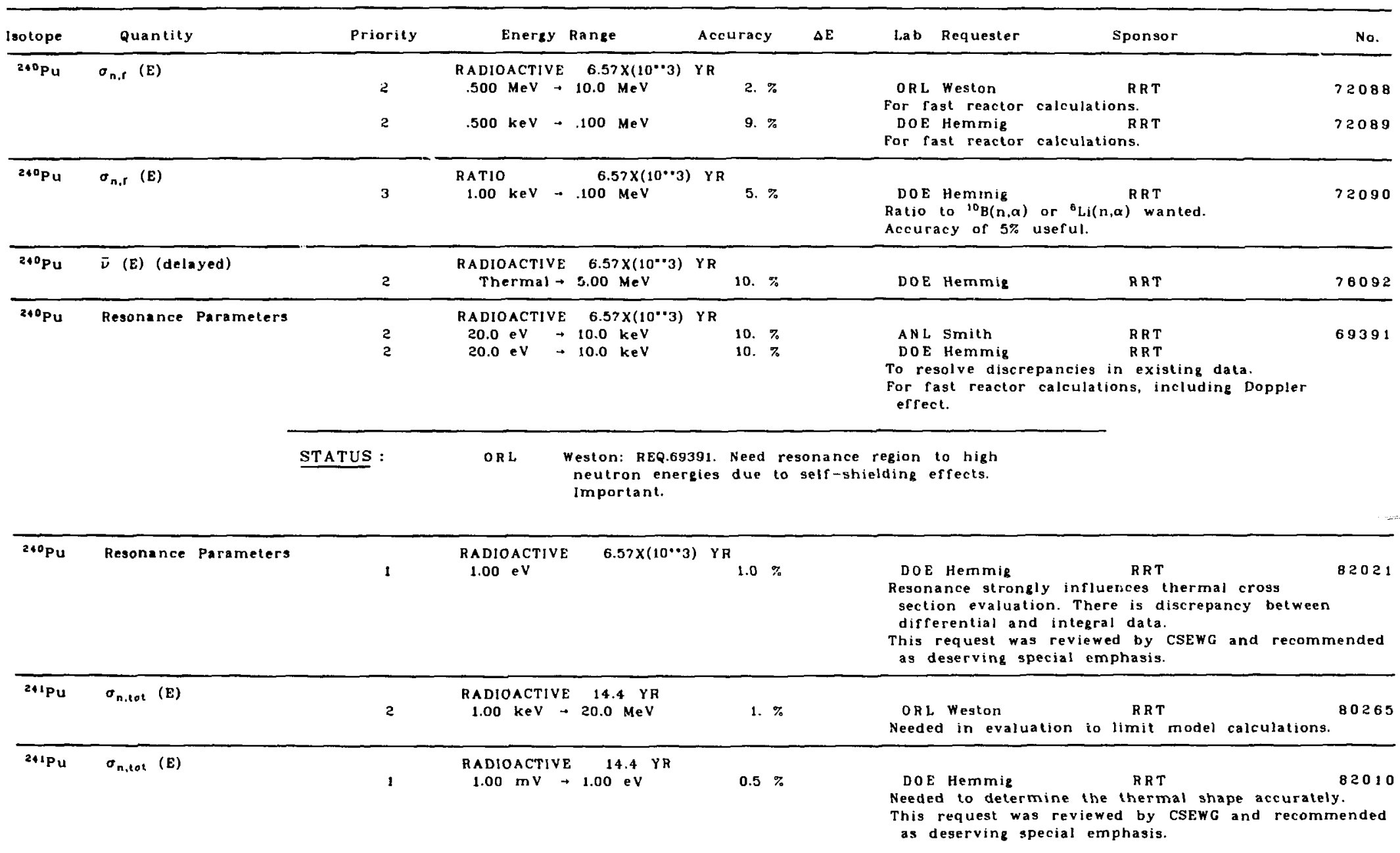


DOE-NDC Compilation of Requests for Nuclear Data 1982

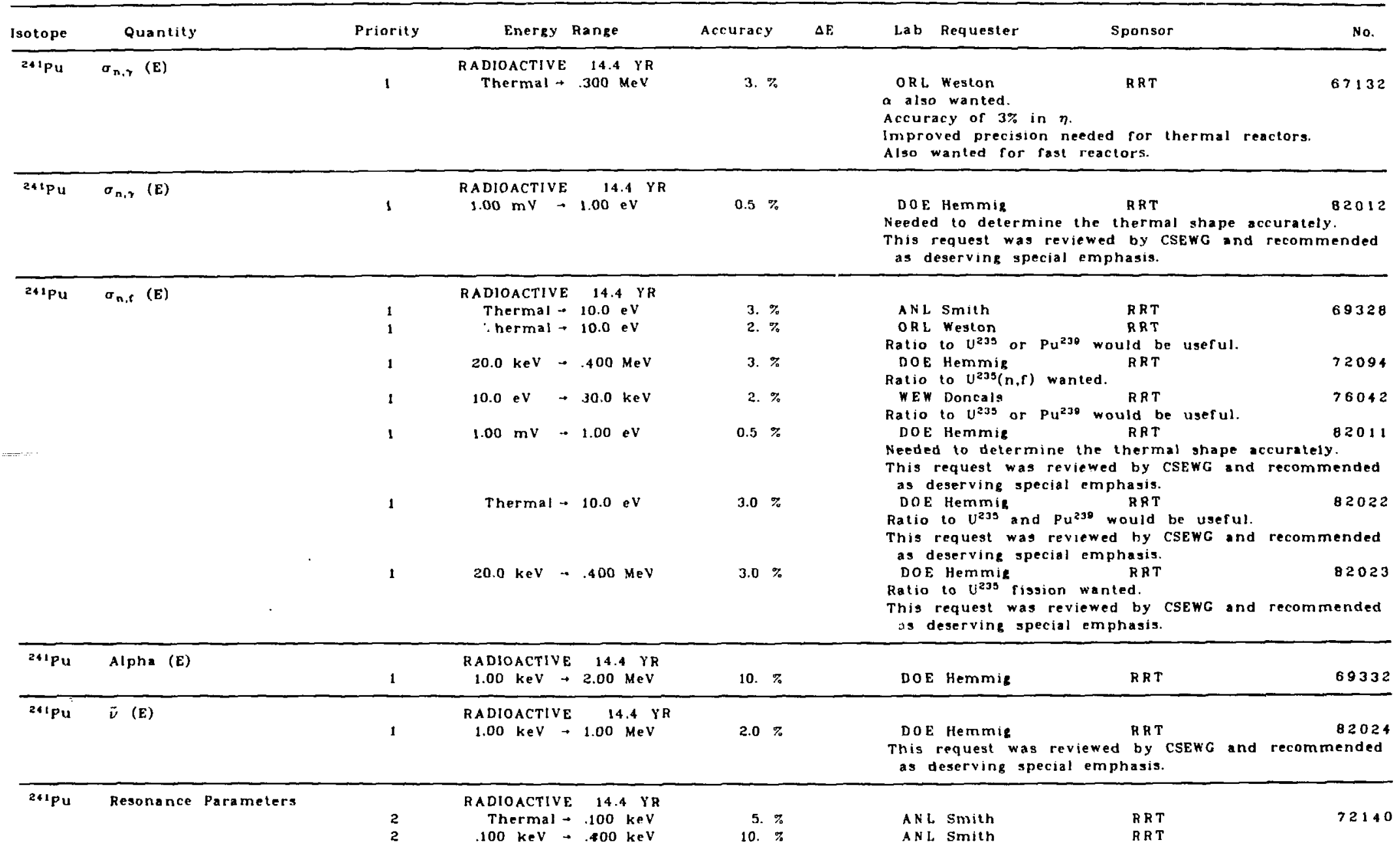


DOE-NDC Compilation of Requests for Nuclear Data 1982

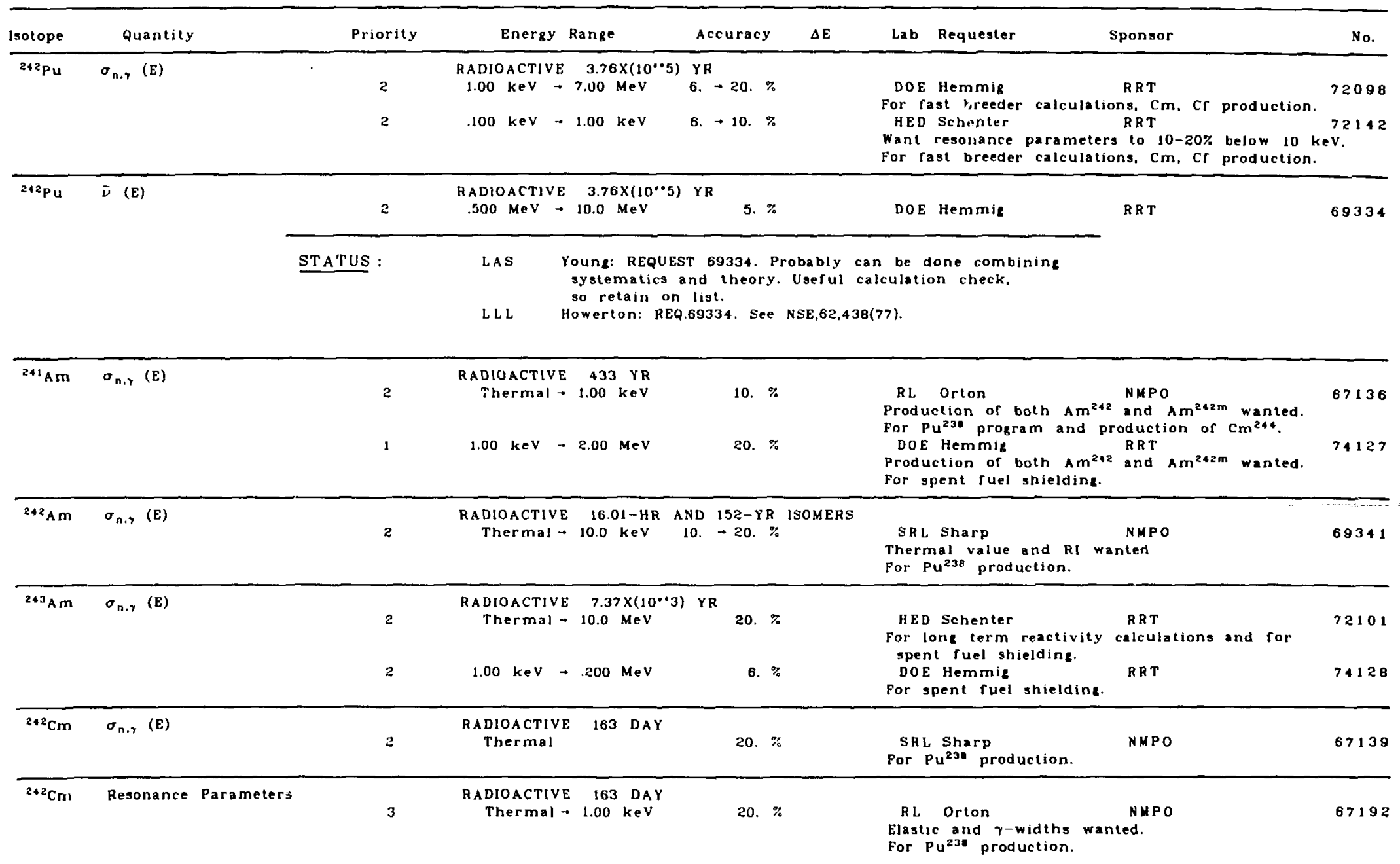


DOE-NDC Compilation of Requests for Nuclear Data 1982

\begin{tabular}{|c|c|c|c|c|c|c|c|c|}
\hline Isotope & Quantity & Priority & Energy & Range & Accuracy & $\Delta \mathrm{E}$ & Lab Requester & No. \\
\hline${ }^{246} \mathrm{Cm}$ & $\sigma_{n, 101}(E)$ & 2 & $\begin{array}{c}\text { RADIOACTIVE } \\
\text { Thermal } \rightarrow\end{array}$ & $\begin{array}{l}4.7 \times\left(10^{--3}\right) \\
10.0 \mathrm{keV}\end{array}$ & Y R & & $\begin{array}{l}\text { SRL. Sharp } \\
\text { Shape of thermal cross section especially } \\
\text { important. } \\
\text { Resonance structure needed. } \\
\text { Accuracy of } 10 \% \text { in RI. }\end{array}$ & 67146 \\
\hline $248 \mathrm{Cm}$ & $\sigma_{n, \gamma}(E)$ & 2 & $\begin{array}{c}\text { RADIOACTIVE } \\
\text { Thermal }\end{array}$ & $\begin{array}{l}4.7 \times\left(10^{*-3}\right) \\
10.0 \mathrm{keV}\end{array}$ & YR 10. $\%$ & & $\begin{array}{l}\text { SRL Sharp } \\
\text { Accuracy of } 10 \% \text { in RI. } \\
\text { To evaluate Cr production. }\end{array}$ & 69350 \\
\hline${ }^{247} \mathrm{Cm}$ & $\sigma_{n, \gamma}(E)$ & 2 & $\begin{array}{c}\text { RADIOACTIVE } \\
\text { Thermal }\end{array}$ & $\begin{array}{l}1.6 \times\left(10^{*} 7\right) \\
10.0 \mathrm{keV}\end{array}$ & $\begin{array}{r}\text { YR } \\
5 .\end{array}$ & & $\begin{array}{l}\text { SRL Sharp } \\
\text { Shape of thermal cross section especially } \\
\text { important. } \\
\text { Accuracy of } 5-10 \% \text { in thermal value and RI. } \\
\text { To evaluate Cr production. }\end{array}$ & 67149 \\
\hline${ }^{247} \mathrm{Cm}$ & $\sigma_{n, s}(E)$ & 2 & $\begin{array}{c}\text { RADIOACTIVE } \\
\text { Thermal }\end{array}$ & $\begin{array}{l}1.6 \times\left(10^{\circ} \cdot 7\right) \\
10.0 \mathrm{keV}\end{array}$ & $\begin{array}{l}\mathrm{YR} \\
5 . \rightarrow 10 . \%\end{array}$ & & $\begin{array}{l}\text { SRL. Sharp } \\
\text { Shape or thermal cross section especially } \\
\text { important. } \\
\text { Accuracy or } 5-10 \% \text { in thermal value and Rl. }\end{array}$ & 67148 \\
\hline${ }^{247} \mathrm{Cm}$ & Resonance Parameters & 2 & $\begin{array}{l}\text { RADIOACTIVE } \\
\text { Thermal - }\end{array}$ & $\begin{array}{l}1.6 \times\left(10^{*} 7\right) \\
10.0 \mathrm{keV}\end{array}$ & Y & & $\begin{array}{l}\text { SRL Sharp } \\
\text { Accuracy or } 20 \% \text { in Rl. } \\
\text { To evaluate Cr production. }\end{array}$ & 67147 \\
\hline${ }^{250} \mathrm{Cr}$ & $\sigma_{n, \gamma}(E)$ & 2 & $\begin{array}{c}\text { RADIOACTIVE } \\
\text { Thermal }\end{array}$ & $\begin{array}{r}13.1 \mathrm{YR} \\
10.0 \mathrm{keV}\end{array}$ & 10. $\%$ & & $\begin{array}{l}\text { SRL Sharp } \\
\text { Accuracy of } 10 \% \text { in RI. } \\
\text { To evaluate Cf production. }\end{array}$ & 69357 \\
\hline${ }^{250} \mathrm{Cr}$ & $\sigma_{n, f}(E)$ & $z$ & $\begin{array}{l}\text { RA DloACTIVE } \\
\text { Thermal }\end{array}$ & $\begin{array}{r}13.1 \mathrm{YR} \\
10.0 \mathrm{keV}\end{array}$ & 10. $\%$ & & $\begin{array}{l}\text { SRL Sharp } \\
\text { Accuracy of } 10 \% \text { in RI. } \\
\text { To evaluate Cr production. }\end{array}$ & 67153 \\
\hline
\end{tabular}


DOE-NDC Compilation of Requests for Nuclear Data 1982

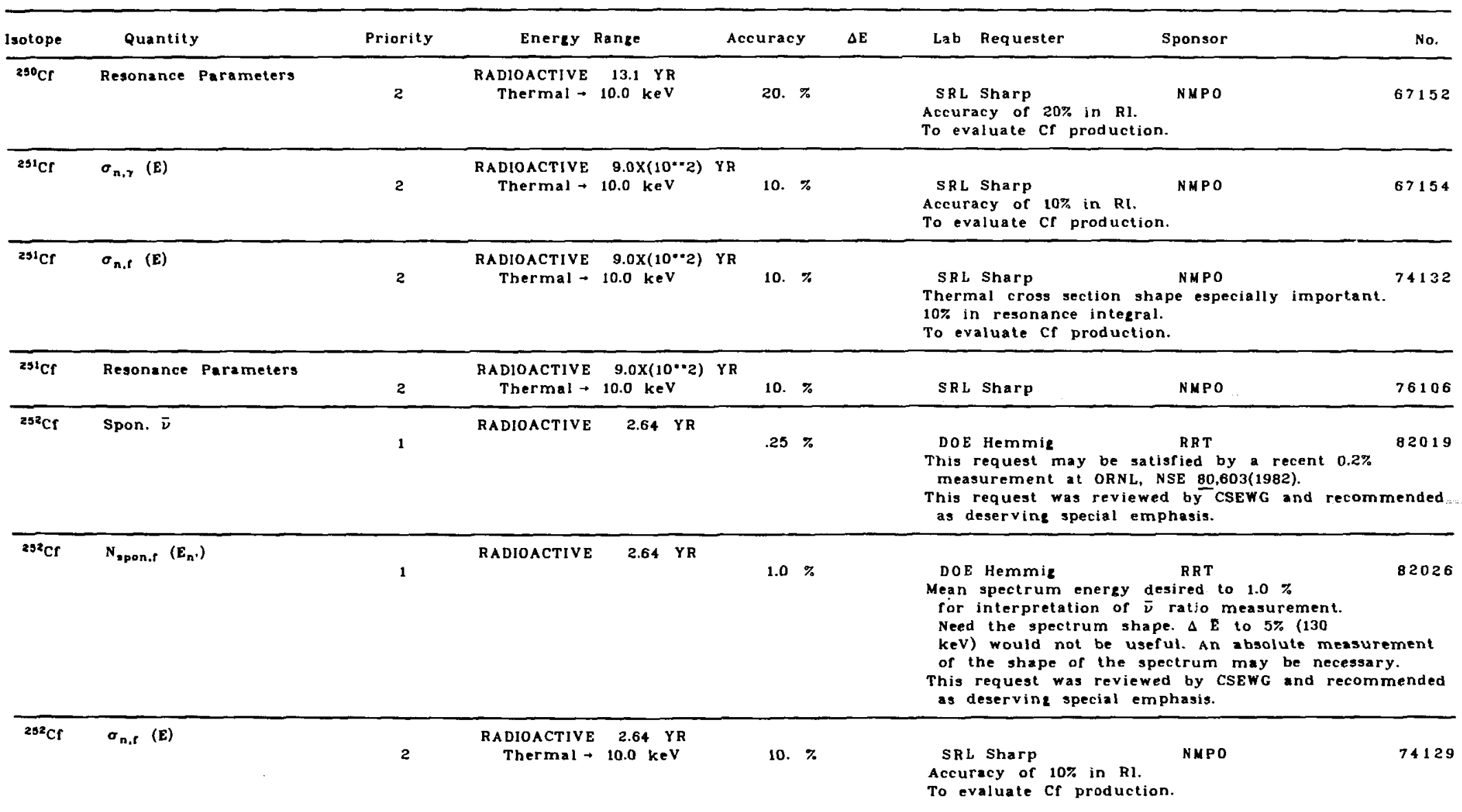




\section{REACTION TABLES FOR DOE-NDC REQUEST LIST}

\section{QUANTITY SYMBOL}

A. Structure Information

Half-life

Half-life (alpha decay)

Halr-life (rission)

spon. $\bar{\nu}$

Prompt $\vec{v}$

$N_{\text {spon.t }}\left(E_{n}\right)$

B. Gamma-ray interactions

$\sigma_{r, n}(\mathrm{E})$

C. Neutron Interactions

$$
\begin{aligned}
& \sigma_{\text {n.tot }} \text { (E) } \\
& \sigma_{n, n} \text { (E) } \\
& \sigma_{n, n}(E, \theta) \\
& \sigma_{n, n} \text { (E) } \\
& \sigma_{n, n^{\prime}}(E, \theta) \\
& \sigma_{n, n^{\prime}}\left(E, E_{n}\right) \\
& \sigma_{n, n^{\prime}} \quad\left(E, \theta, E_{n} .\right) \\
& \sigma_{n, 3 c e} \text { (E) } \\
& \sigma_{\text {n.non }}(E) \\
& \sigma_{n, \bullet b s} \text { (E) } \\
& \sigma_{n, \gamma} \text { (E) } \\
& N_{n, \gamma}^{n, \gamma}\left(E, E_{\gamma}\right) \\
& \sigma_{n, n \cdot \gamma}\left(E, \theta_{\gamma}\right) \\
& \sigma_{n, x \gamma} \text { (E) } \\
& \sigma_{n . x_{\gamma}}\left(E, E_{\gamma}\right) \\
& \sigma_{n, X_{\gamma}}\left(E, \theta_{\gamma}, E_{\gamma}\right) \\
& \sigma_{n, 2 n}(E) \\
& \sigma_{n, 3 n} \text { (E) } \\
& \sigma_{n, 4 n} \text { (E) } \\
& \sigma_{n, 5 n} \text { (E) } \\
& \sigma_{n, x_{n}} \text { (E) } \\
& \sigma_{n, n_{n}} \quad\left(E, E_{n}\right) \\
& \sigma_{n, \times n}\left(E, \theta, E_{n}\right) \\
& \sigma_{n, p} \text { (E) } \\
& \sigma_{n, n ' p}(E) \\
& \sigma_{n, n \cdot 2 p} \text { (E) } \\
& \sigma_{n, 2 p} \text { (E) }
\end{aligned}
$$

\section{DEFINITION}

Half life

Alpha half life

Fission half life

Average number of neutrons emitted per fission

Average number of prompt neutrons per fission

Energy distribution of fission neutrons

Neutron production cross section

Proton production cross section

Total cross section

Elastic cross section

Angular distribution of elastically scattered particles

Inelastic cross section

Angular distribution of inelastically scattered particles

Energy distribution of inelastic particles

Double differential (energy,angle) inelastic cross section

Total scattering cross section (elastic + inelastic)

Non-elastic cross section

Absorption cross section

Capture cross section

Capture gamma ray spectra

Angular distribution of photons from inelastic scattering

Total photon production cross section from all photon-emitting processes

Energy distribution of photons from all photon emitting processes

Double differential (energyangle) total photon emission cross section

Two neutron production cross section

Three neutron production cross section

Four neutron production cross section

Five neutron production cross section

Total neutron production cross section from ali neutron emitting processes

Energy distribution of neutrons from all neutron emitting processes

Double differential (energy,angle) neutron emission cross section

Proton production cross section

Neutron and proton production cross section

Neutron and 2-proton production cross section

Two proton production 


\section{QUANTITY SYMBOL}

\section{Neutron Interactions}

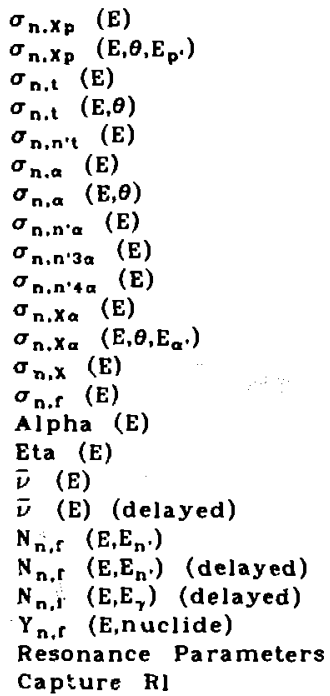

D. Proton Interactions

$$
\begin{array}{ll}
\sigma_{p, \gamma} & (E) \\
\sigma_{p, n} & (E) \\
\sigma_{p, p} & (E) \\
\sigma_{p, 3 a}(E) & \left(E, \theta, E_{a}\right)
\end{array}
$$

E. Deuteron Interactions

$$
\begin{array}{ll}
\sigma_{d, 2 n} & (E) \\
\sigma_{d, p} & (E) \\
\sigma_{d, d} & (E) \\
\sigma_{d, a} & (E)
\end{array}
$$

\section{DEFINITION}

Total proton production cross section from all processes

Double differential (energy,angle) proton emission cross section Triton production cross section

Angular distribution of tritons

Neutron and triton production cross section

Alpha production cross section

Angular distribution of alpha particles

Neutron and alpha production cross section

Emission of a neutron and three alpha particles

Emission of a neutron and four alpha particles

Total alpha production cross section from all processes

Double differential (enerey,angle) alpha emission cross section

special quantity further defined in comments

Fission cross section

Capture to fission ratio e.e. $\sigma(\mathbf{n}, \boldsymbol{\gamma}) / \sigma(\mathbf{n}, \mathbf{f})$

Number of neutrons emitted per neutron absorption

Average number of neutrons emitted per fission

Average number of delayed neutrons emitted per figsion

Energy distribution of fission neutrons

Energy distribution of delayed fission neutron:

Spectrum of delayed samma rays emitted in fission

Fission product mass yield spectrum

Resonance parameters

Capture resonance integral

Capture cross section

Neutron production cross section

Proton production cross section

Production of three alpha particles

Double differential (energy,angle) alpha emission cross section

Two neutron production cross section

Proton production cross section

Deuteron production cross section

Alpha production cross section 
APPENDIX A

QUANTITY SYMBOL

F. Triton Interactions

$\sigma_{2,3 n}(\mathrm{E})$

$\sigma_{\mathrm{t}, \mathrm{t}}(\mathrm{E})$

$\sigma_{t, a}(\mathrm{E})$

G. Helium-3 interactions

$\sigma_{H e-3, p}(E)$

$\sigma_{H e-3,2,}$ (E)

$\sigma_{\text {He-3.He-3 (E) }}$

$\sigma_{H e-3, X}$ (E)

H. Alpha interactions

$\sigma_{a, a} \quad(E)$

$\sigma_{a, n}(E)$

$\sigma_{a, p}(\mathrm{E})$

J. Lithium-6 Interactions

$\sigma_{L i-6, X}(\mathrm{E})$
DEFINITION

Three neutron production cross section

Triton production cross section

Alpha praduction cross section

Proton production cross section

Two proton production

Helium-3 production cross section

special quantity further defined in comments

Elastic cross section

Neutron production cross section

production cross section

Special quantity further defined in comments 
LIST OF REQUESTERS

\section{NAME}

D. Bartine

S. E. Berk

C. D. Bowman

A. D. Carlson

L. L. Carter

R. S. Caswell

c. L. Cowan

D. E. Dei

M. Divadeenam
LAB

OR L

R R T

DOE

OFE

$301-353-417$

$505-667-1757$

LAS NBS

N B S

R R

$301-921-2677$

HED

OFE

$509-376-5193$

N B S

OHE R

$301-921-2625$

C B

R R T

D N R

R T

OFE

$615-483-6897$

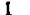




\section{NAME}

R. C. Doncals

D. E. Driemeyer

C. Eisenhauer

B. Engholm

F. Feiner

c. Y. Fu

D. M. Gilliam

L. Green

R. C. Haight

G. Hale
LAB AGENCY

R R T

FE

D N R

K A

O R L

$\mathrm{RRT}$

DNA
OFE

N B S

N B S

$301-921-2677$

OFE

$412-722-5381$

$415-411-4510$

$505-667-7738$

\section{ADDRESS}

Westinghouse Electric Corporation

Advanced Reactor Division

Waltz Mill Site

P. O. Box 158

Madison, PA 15663

McDonnell Douglas Astronautics Company St. Louis Division

Box 516

St. Louis, MO 63166

National Bureau of Standards

Washington. DC 20234

\section{General Atomic}

P.0, Box 81608

San Diego, CA 92138

Knolls Atomic Power Laboratory

P. O. Box 1072

Schenectady, NY 12301

Oak Ridge National Laboratory

Neutron Physics Division

P. O. Box $X$

Oak Ridge, TN 37830

National Bureau of Standards

Washington. DC 20234

Westinghouse Electric Corporation Advanced Reactor Division

Waltz Mill Site

P. O. Box 158

Madison, PA 15663

Lawrence Livermore National Laboratory

P. 0. Box 808

Livermore, CA 94550

Los Alamos National Laboratory

P. O. Box 1663

Los Alamos, NM 87545 


\section{NAME}

P. B. Hemmig

D. Larson

B. R. Leonard, $\mathrm{J} r$.

F. M. Mann

W. N. McElroy

E. Dale McGarry

S. F. Mughabghab

D. W. Muir

G. T, Orton

R. w. Peelle

w. P. Poenitz
$615-574-6119$

\section{PHONE}

$301-353-3577$

$509-946-2558$

$509-376-5598$

$509-942-3791$

$301-921-2421$

$516-282-2902$

$505-667-1207$

$509-942-7465$

$312-972-2816$

\section{A DDRESS}

U. S. Department of Energy Division of Reactor Research and Technology

Washington, DC 20545

oak Ridge National Laboratory Neutron Physics Building P. O. Box $X$

Oak Ridge, TN 37830

Pacific Northwest Laboratory

P. O. Box 999

Richland, WA 99352

Hanford Enginecring Development Laboratory P. O. Box 1970

Richland, WA 99352

Hanford Engineering Development Laboratory P. O. Box 1970

Richland, WA 99352

National Bureau of Standards Nuclear Radiation Division

Washington, DC 20234

National Nuclear Data Center Brookhaven National Laboratory Upton. NY 11973

Los Alamos National Laboratory P. O. Box 1663

Los Alamos, NM 87545

Richland Operations office

P. O. Box 550

Richland, WA 9935

Oak Ridge National Laboratory

P. O. BoX $X$

Oak Ridge, TN 37830

Argonne National Laboratory 9700 South Cass Avenue

Argonne, IL 60439 
APPENDIX B

\section{NAME}

A. Prince

J. A. Rawlins

R. E. Schenter

D. A. Sharp

A. B. Smith

D. Steiner

L. Stewart

R. B. Walton

L. Weston

P. G. Young

\section{LAB AGENCY}

BNL RRT

H E D

RRT

H E D

R R T

N M P O

$803-725-2373$

$312-972-6084$

R R T

OF E

$518-270-6407$

$O M A$

RRT

LAS

D S S

O R L

RRT

L A S

PHONE

$516-282-5079$

$509-376-3314$

$509-376-3339$

$505-667-7737$

$505-667-4393$

$615-574-6129$

OMA

OFE

\section{ADDRESS}

Brookhaven National Laboratory Upton, NY 11973

Hanford Engineering Development Laboratory P. D. Box 1970

Richland, WA 99352

Hanford Engineering Development Laboratory

P. O. Box 1970

Richland. WA 99352

Savannah River Laboratory

P. O. Box 117

Aiken, SC 29801

Argonne National Laboratory

Applied Physics Division

9700 South Cass Ave.

Argonne, IL 60439

Rensselaer Polytechnic Institute

Department of Nuclear Engineering

Troy, NY 1218

Los Alamos National Laboratory

P O. Box 1663

Los Alamos, NM 87545

Los Alamos National Laborator

P. O. Box 1663

Los Alamos, NM 87545

Oak Ridge National Laboratory

P. O. BoX X

Oak Ridge, TN 37830

Los Alamos National Laboratory

P. O. BoX 1663

Los Alamos, NM 87545 
LIST OF MATERIALS AND EVALUATORS

\begin{tabular}{|c|c|c|}
\hline Material & Principal Evaluator(s) & Laboratory \\
\hline $1-H-1$ & L.stewart & LAS \\
\hline $1-\mathrm{H}-\mathrm{Z}$ & L.Stewart & LAS \\
\hline $1-\mathrm{H}-3$ & L.Stewart & LAS \\
\hline $2-\mathrm{He}-3$ & L.Stewart & LAS \\
\hline $2-\mathrm{He}-4$ & G.M.Hale & LAS \\
\hline $3-L i-6$ & G.M.Hale & LAS \\
\hline $3-\mathrm{Li}-7$ & R.J.LaBauve & LAS \\
\hline $4-\mathrm{Be}-9$ & R.J.Howerton & LLL \\
\hline $5-B-10$ & G.M.Hale & LAS \\
\hline $5-B-11$ & C.Cowan & GEB \\
\hline $6-\mathrm{C}$ & C.Y.Fu & ORL \\
\hline $7-N-14$ & P.G.Young & LAS \\
\hline $7-N-15$ & E.D.Arthur & LAS \\
\hline $8-0-16$ & P.G.Young & LAS \\
\hline $8-0-17$ & B.A.Magurno & BNL \\
\hline $9-F-19$ & C.Y.Fu & ORL \\
\hline $11-\mathrm{Na}-23$ & D.C.Larson & ORL \\
\hline $12-M_{2}$ & D.C.Larson & ORL \\
\hline $13-A 1-27$ & P.G.Young & LAS \\
\hline $14-\mathrm{Si}$ & D.C.Larson & ORL \\
\hline $15-P-31$ & R.J.Howerton & LLL \\
\hline $16-5$ & M.Divadeenam & BNL \\
\hline $16-s-32$ & R.J.Howerton & LLL \\
\hline $17-\mathrm{Cl}$ & M.K.Drake & SAI \\
\hline $19-K$ & M.K.Drake & SAI \\
\hline $20-\mathrm{Ca}$ & C.Y.Fu,F.G.Perey & ORL \\
\hline $22-\mathrm{Ti}$ & C.Philis,A.Smith,R.Howerton & BRC, ANL,LLL \\
\hline $23-v$ & A.Smith,H.Howerton,F.Mann & ANL,LLL,HED \\
\hline $24-\mathrm{Cr}$ & A.Prince & BNL \\
\hline $25-M n-55$ & S.F.Mughabghab & BNL \\
\hline $26-\mathrm{Fe}$ & C.Y.Fu & ORL \\
\hline $27-\mathrm{Co}-59$ & S.F.Mughabghab & BNL \\
\hline $28-\mathrm{Ni}$ & M.Divadeenam & BNL \\
\hline $29-\mathrm{Cu}$ & C.Y.Fu & ORL \\
\hline $31-G \mathbf{a}$ & R.G.Howerton,P.G.Young & LLL,LAS \\
\hline $36-\mathrm{Kr}$ & A.Prince & BNL \\
\hline $40-\mathrm{Zr}$ & M.Drake & $\mathbf{S A I}_{\mathbf{I}}$ \\
\hline 42-Mo & R.J.Howerton,F.Schmittroth & LLL,HED \\
\hline 43-Tc-99 & R.Schenter,Z.Livolsi & HED, $B+W$ \\
\hline $45-R h-103$ & R.Schenter,Z.Livolsi & HED, B + W \\
\hline
\end{tabular}

\begin{tabular}{|c|c|c|}
\hline Material & Principal Evaluator(s) & iaboratory \\
\hline $47-A g-107$ & R.Schenter,M.Bhat & HED.BNL \\
\hline $47-A g-109$ & R.Schenter,M.Bhat & HED, BNL \\
\hline $48-\mathrm{Cd}$ & S.Pearlstein & BNL \\
\hline $48-C d-113$ & S.Pearlstein,F.Mann & BN L,HED \\
\hline $54-X e$ & M.R.Bhat, S.F.Mughabghab & BNL \\
\hline $54-X e-135$ & B.R.Leonard,Jr. & BN W \\
\hline $55-C s-133$ & R.Schenter,M.Bhat & HED,BNL \\
\hline $56-B a-138$ & R.J.Howerton & LLL \\
\hline $62-5 m-149$ & R.Schenter,B.R.Leonard,Jr. & HED,BNW \\
\hline $63-E \mathbf{u}$ & S.F.Mughabghab & BNL \\
\hline $64-G d$ & B. A.Magurno & BNL \\
\hline $66-D y-164$ & B.R.Leonard,Jr. & BN W \\
\hline $71-L u$ & B.R.Leonard,Jr. & BNW \\
\hline $72-\mathrm{Hf}$ & M.K.Drake & SAl \\
\hline $73-T a-181$ & R.J.Howerton & LLL \\
\hline $73-\mathrm{Ta}-182$ & J.otter & A. I \\
\hline $74-W$ & J.Otter & AI \\
\hline 75-Re & W.B.Henderson & WAP \\
\hline $79-A u-197$ & S.F.Mughabghab & BNL \\
\hline $82-\mathrm{Pb}$ & C.Y.Fu & ORL \\
\hline $83-8 i-209$ & D.Smith,R.J.Howerton & ANL,LLL \\
\hline $90-T h-232$ & Bhat,Smith,Leonard,DeSaussure & BNL, ANL, BNW,ORL \\
\hline $91-P a-233$ & L.F.Mann,C.Reich & HED,INL \\
\hline $92-U-233$ & L.Stewart,L.Weston,F.Mann & LAS,ORL,HED \\
\hline $92-U-234$ & M.Divadeenam,F.Mann,C.Reich & BNL,HED \\
\hline $92-U-235$ & M.R.Bhat & BN L \\
\hline $92-U-236$ & M.Divadeenam,F.Mann,C.Reich & BNL,HED \\
\hline $92-U-238$ & E.Pennington & ANL \\
\hline $93-N p-237$ & F.Mann,R.Ben jamin,A.Smith,C.Reich & HED,SR L, AN L, INL \\
\hline $94-P u-238$ & F.Mann,C.Dunford & HED,BNL \\
\hline $94-P u-239$ & E.Kuja wski,L.Stewart & GEB,LAS \\
\hline $94-P u-240$ & L.W.Weston & ORL \\
\hline $94-P u-241$ & L.W.Weston,R.J.Howerton & ORL,LLL \\
\hline $94-P u-242$ & Mann,Benjamin, Madland, Howerton & HED,SRL,LAS,LLL \\
\hline $95-A m-241$ & F.Mann,L.W.Weston & HED,ORL \\
\hline $95-A m-242 m$ & F.Mann.R.Ben jamin.R.J.Howerton & HFD,SRL,LLL \\
\hline $95-A m-243$ & F.Mann,R.Benjamin,R.J.Howerton & HED,SRL,LLL \\
\hline $96-C m-243$ & F.Mann,R.Ben jam in,R.J.Howerton & HED,SRL, LLL \\
\hline $96-\mathrm{Cm}-244$ & F.Mann,R.Ben jamin,R.J.Howerton & HED,SRL, LLL \\
\hline $96-\mathrm{cm}-245$ & R.W.Ben jamin,R.J.Howerton & SRL,LLL \\
\hline $96-\mathrm{cm}-246$ & R.Ben jamin,R.Howerton,R.Kinsey & SRL,LLL,BNL \\
\hline
\end{tabular}

